\title{
Ferroelectric thin films: Review of materials, properties, and applications
}

N. Setter, D. Damjanovic, L. Eng, G. Fox, S. Gevorgian, S. Hong, A. Kingon, H. Kohlstedt, N. Y. Park, G. B. Stephenson, I. Stolitchnov, A. K. Taganstev, D. V. Taylor, T. Yamada, and S. Streiffer

Citation: Journal of Applied Physics 100, 051606 (2006);

View online: https://doi.org/10.1063/1.2336999

View Table of Contents: http://aip.scitation.org/toc/jap/100/5

Published by the American Institute of Physics

\section{Articles you may be interested in}

Ferroelectricity in hafnium oxide thin films

Applied Physics Letters 99, 102903 (2011); 10.1063/1.3634052

Ferroelectric or non-ferroelectric: Why so many materials exhibit "ferroelectricity" on the nanoscale Applied Physics Reviews 4, 021302 (2017); 10.1063/1.4979015

Ferroelectric, pyroelectric, and piezoelectric properties of a photovoltaic perovskite oxide Applied Physics Letters 110, 063903 (2017); 10.1063/1.4974735

Ultrahigh strain and piezoelectric behavior in relaxor based ferroelectric single crystals Journal of Applied Physics 82, 1804 (1998); 10.1063/1.365983

Depolarization fields in thin ferroelectric films Journal of Applied Physics 44, 3379 (2003); 10.1063/1.1662770

Multiferroic magnetoelectric composites: Historical perspective, status, and future directions Journal of Applied Physics 103, 031101 (2008); 10.1063/1.2836410

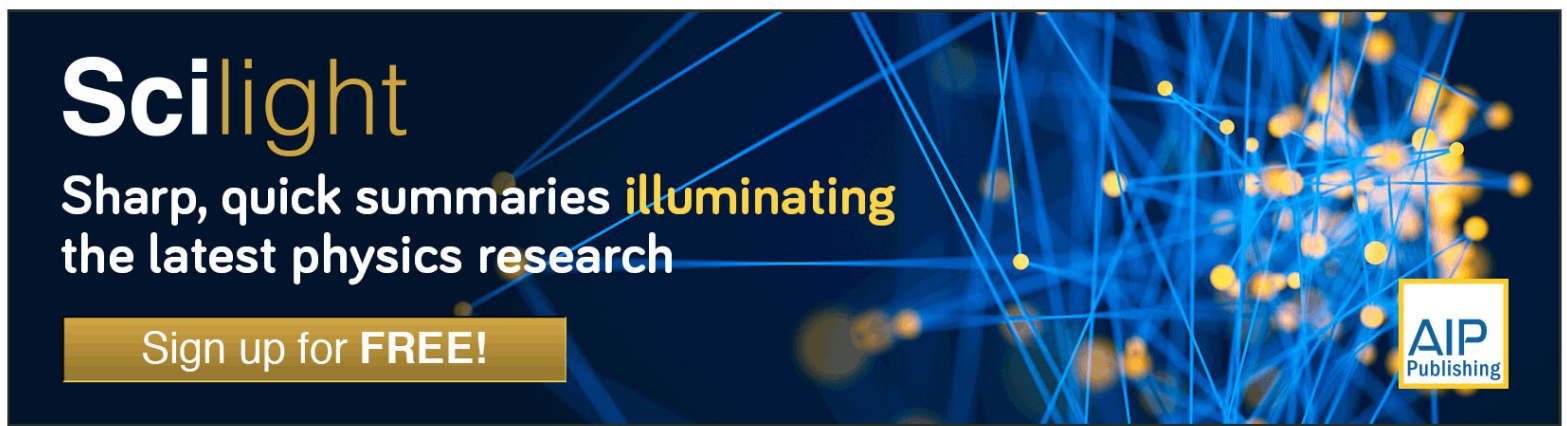




\title{
Ferroelectric thin films: Review of materials, properties, and applications
}

\author{
N. Setter ${ }^{\mathrm{a})}$ and D. Damjanovic \\ Ceramics Laboratory, Swiss Federal Institute of Technology (EPFL), CH-1015 Lausanne, Switzerland \\ L. Eng \\ Institute of Applied Physics/Photophysics, University of Technology Dresden, Dresden D-01062, Germany \\ G. Fox \\ Ramtron Int. Corp., Colorado Springs, Colorado 80921 \\ S. Gevorgian \\ Department of Microtechnology and Nanoscience, Chalmers University of Technology, \\ SE-41296 Gothenburg, Sweden and Microwave and High Speed Electronics Research Center, \\ Ericsson AB, 43184 Moelndal, Sweden \\ S. Hong \\ Nano Devices Laboratory, Samsung Advanced Institute of Technology, Suwon, 440-600, Korea
}

\section{A. Kingon}

Department of Materials Science and Engineering, North Carolina State University, Raleigh, North Carolina 27695-7919

H. Kohlstedt ${ }^{\text {b) }}$

Institut für Festkörperforschung (IFF), Forschungszentrum Jülich, 52425 Jülich, Germany

N. Y. Park

Nano Devices Laboratory, Samsung Advanced Institute of Technology, Suwon, 440-600, Korea

G. B. Stephenson

Materials Science Division, Argonne National Laboratory, Argonne, Illinois 60439

I. Stolitchnov, A. K. Taganstev, D. V. Taylor, ${ }^{\text {c) }}$ and T. Yamada

Ceramics Laboratory, Swiss Federal Institute of Technology, EPFL, CH-1015 Lausanne, Switzerland

\section{S. Streiffer}

Materials Science Division, Argonne National Laboratory, Argonne, Illinois 60439

(Received 12 December 2005; accepted 31 January 2006; published online 12 September 2006)

\begin{abstract}
An overview of the state of art in ferroelectric thin films is presented. First, we review applications: microsystems' applications, applications in high frequency electronics, and memories based on ferroelectric materials. The second section deals with materials, structure (domains, in particular), and size effects. Properties of thin films that are important for applications are then addressed: polarization reversal and properties related to the reliability of ferroelectric memories, piezoelectric nonlinearity of ferroelectric films which is relevant to microsystems' applications, and permittivity and loss in ferroelectric films-important in all applications and essential in high frequency devices. In the context of properties we also discuss nanoscale probing of ferroelectrics. Finally, we comment on two important emerging topics: multiferroic materials and ferroelectric one-dimensional nanostructures. (C) 2006 American Institute of Physics. [DOI: 10.1063/1.2336999]
\end{abstract}

\section{INTRODUCTION}

Ferroelectrics are essential components in a wide spectrum of applications. In thin film form, ferroelectrics and, more widely, polar materials have now been used for several years in rf devices and in nonvolatile memories. Components based on ferroelectric films are also being developed for various sensor and actuator applications and for tunable microwave circuits.

The development of ferroelectric thin films started at the

\footnotetext{
a)Electronic mail: nava.setter@epfl.ch

${ }^{b)}$ Present address: Department of Materials Science and Engineering and Department of Physics, University of California, Berkeley, California 94720.

${ }^{c}$ Present address: Nanosys Inc., Palo Alto, California 94304.
}

late 1960s and early 1970s when progress in integrated Si devices and thin film processing techniques triggered interest in the use of ferroelectric thin films for fabrication of nonvolatile memories. ${ }^{1-3}$ Difficulties with ferroelectric materials processing and integration frustrated the attempts to make practical memory devices until the 1980s. The advances in processing of complex ferroelectric oxides at the mid-1980s, ${ }^{4,5}$ and perhaps also the discovery of the high temperature superconductive oxides and the subsequent efforts to integrate them onto silicon resulted in a revival of interest in ferroelectric memories, and in 1987, ferroelectric memory integrated with silicon complementary metal-oxide semiconductor (CMOS) was demonstrated. ${ }^{6}$ At about the same time first steps were made towards the integration of microsensors 
with microelectronics and the area of micro electro mechanical systems (MEMSs) emerged. Soon it was realized that ferroelectric films could add functionality, e.g., sensing functions, to MEMSs. During the late 1990s, portable telephones became a mass product and the introduction of polar thin films contributed, during the past five years, to their further miniaturization and improved performance. Ferroelectric films are now being used in a number of commercial, wide spread products; these applications and those under development include memories, microwave electronic components, and microdevices with pyroelectric and piezoelectric microsensors/actuators.

Over the past 15 years the applications have triggered intense research toward understanding materials and physics issues that govern the functioning of the films in devices. Several books, ${ }^{7-9}$ journal publications, ${ }^{10}$ and recent reviews ${ }^{11,12}$ present the knowledge gained in the field. Understanding the origin of the differences between properties of films and those of massive ferroelectrics is advancing but open questions persist: for example, the origin of the higher dielectric losses and lower maximum permittivity of these films is not fully understood. Thin films lag behind bulk ferroelectrics in materials development, e.g., relaxor ferroelectrics and lead-free materials. Domain engineering, an area under intense investigations in bulk ferroelectrics, has been hardly examined in thin films.

As an introduction to the research papers in this special issue on ferroelectric thin films, we set the target of reviewing the state of art in the field. During the writing we had to proportionate this ambitious goal and reviewed only selected topics in this large field. Firstly, we present the state of art in applications and potential applications of ferroelectric films, reviewing the achievements and challenges as of today. We continue with a chapter on materials, structures (domains, in particular), and size effects. Then, we review the present understanding of selected properties of ferroelectric thin films that are of a high importance in device applications: properties related to the reliability of ferroelectric memories, piezoelectric nonlinearity of ferroelectric films which is relevant to MEMS applications, and permittivity and loss in ferroelectric films-important in all applications, but particularly important in high frequency devices. In the final section we address briefly emerging topics: multiferroics and onedimensional (1D) ferroelectric nanostructures. Deeper insight into current research topics can be found in the papers that follow in this "Ferroelectric Thin Films" issue.

\section{FERROELECTRIC FILM BASED COMPONENTS AND DEVICES}

The three main domains of applications of ferroelectric films are microsystems (microsensor arrays, etc.), memories, and high frequency electrical components. These domains are reviewed below. First, we summarize the situation in sensor and actuator applications using piezoelectric films. Then, applications in high frequency communications are discussed: firstly, polar films in rf filters and then tunable applications in microwave electronics. Finally, a brief review of the ferroelectric random access memory (FRAM) state of art is followed by an overview of alternative memories. In each case, issues specific to the given application are briefly addressed as well: clamping of the films-in piezoelectric applications in MEMSs, thermal stability in tunable ferroelectrics, and reliability issues in FRAMs. Properties and functionality are discussed in more details in Sec. IV of this review.

\section{A. Piezoelectric microsensors and microactuators}

Miniature systems containing devices or arrays of devices that combine electronics with other components such as sensors, transducers, and actuators, and which are fabricated by integrated circuit (IC) batch processing techniques, constitute the MEMS domain. Often the substrates are micromachined (bulk micromachining) or the elements are suspended above the substrate (surface micromachining), which is the main difference in comparison with standard IC technology.

Deposition of ferroelectric films on silicon or other substrates prior to the microfabrication is an attractive way to add sensing and actuating functionalities to MEMSs. In spite of the additional complexity of fabrication, piezoelectric film based devices are attracting attention. ${ }^{13}$ The advantages of piezoelectrics are a high generated force and good actuating range, a wide frequency range of operation, and a low power consumption. Pyroelectric microarrays have been developed too for a number of sensor and imaging applications. ${ }^{14}$

The fabrication technology of ferroelectric MEMSs poses some different challenges from those encountered in ferroelectric based microelectronic devices: the piezoelectric layers are typically of a higher thickness (typically $0.5-5 \mu \mathrm{m}$ ) which necessitates longer annealing periods at high temperatures. The materials of choice for piezoelectric actuator and transducer applications and for pyroelectric applications are $\mathrm{Pb}(\mathrm{Zr}, \mathrm{Ti}) \mathrm{O}_{3}$ ( $\mathrm{PZT}$ ) near the morphotropic boundary and $\mathrm{PbTiO}_{3}$, respectively. The preferred deposition method is the sol-gel route, especially in light of the lower cost of the process, which is required due to the typically small scale of production in MEMSs relative to microelectronics. The textured growth, which is necessary for optimizing properties, is complicated and requires a complete control of the nucleation and growth mechanisms. For example, the textured (100) growth of PZT, which yields the best piezoelectric response, is obtained using a $\mathrm{PbTiO}_{3}$ template layer of a thickness inferior to $10 \mathrm{~nm} .^{15}$

At the same time the structure of cantilevers and membranes, used in most of the applications, is sensitive to residual stresses. The processing procedure has been optimized in the recent five years and a number of academic and industrial groups have mastered the process. Recent reviews on processing of ferroelectric layers and on issues of microfabrication of devices are available. ${ }^{16}$

\section{Piezoelectric based microdevices}

The development of piezoelectric based microdevices started in the 1990s. The various devices which were fabricated, tested, and analyzed for a rather comprehensive evaluation of their potentials and limits include ultrasonic micromotors, ${ }^{17,18}$ micropumps and microvalves, ${ }^{19}$ acceler- 
ometers and gyroscopes, ${ }^{20}$ acoustic sensors, ${ }^{21}$ sensing and actuating elements in atomic force microscope (AFM) cantilevers ${ }^{22}$ rf and optical switches ${ }^{23}$ and ultrasonic transducers for medical ${ }^{24}$ and sonar ${ }^{25}$ applications.

In such devices, PZT has remained the standard thin film material. AlN may be advantageous in sensor applications due to its lower permittivity and dielectric losses, which translate into a better voltage output and signal to noise ratio. PZT is the more suitable material when current output is used and, more important, for actuator and transducer applications in which force, torque, and output power are important. In these cases the high piezoelectric and electromechanical coupling coefficients of PZT are important. Relaxor and relaxor-ferroelectric thin films could potentially be superior to $\mathrm{PZT},{ }^{26,27}$ due to their high piezoelectric coefficients in the latter and nonhysteretic behavior in the former, but then fabrication know-how and their full evaluation need yet to be fully completed.

The most important difference between piezofilms and bulk piezoelectrics is obviously the clamping of the film by the substrate. The clamping results in a reduction of the macroscopically measured $d_{33, f}$ coefficient [Eq. (1)] by approximately half, relative to $d_{33}$, the bulk value, if the $d_{31}$ coefficient has roughly half the value of $d_{33}$ as is the case in PZT. $d_{33, f}$ here is the out-of-plane longitudinal coefficient of a film sandwiched between two electrodes and deposited on a substrate. The index $f$ is added, indicating that the value is not the intrinsic one for the piezoelectric material, since the elastic properties of the substrate and the electrode/piezofilm sandwich intervene in the measured values.

$$
d_{33, f}=d_{33}-\frac{2 s_{13}^{E}}{s_{11}^{E}+s_{12}^{E}} d_{31} \text {. }
$$

This piezoresponse is stronger in local measurement by piezoresponse force microscopy. Furthermore, the $d_{33}$ coefficient is increased when the lateral clamping is released by fabrication of piezoelements with lateral dimensions of $100 \mathrm{~nm}$ or $\operatorname{less}^{28}$ (Fig. 1).

The practical piezoelectric coefficient in thin film applications is often the $e_{31, f}$ coefficient which relates the polarization developed between the electrodes perpendicular to the film surface to the induced in-plane stresses in the film.

$$
e_{31, f}=\frac{d_{31}}{s_{11}^{E}+s_{12}^{E}}=e_{31}-\frac{c_{13}^{E}}{c_{33}^{E}} e_{33} .
$$

Note that the absolute value of $e_{31, f}$ is higher than $e_{31}$. In PZT, the maximum $e_{31, f}$ is obtained in (100) rhombohedral films, not far from the morphotropic boundary. ${ }^{29}$ This is also shown phenomenologically to be the direction of maximal response in monodomain epitaxial thin films with the pseudocubic [001] perpendicular to the substrate. ${ }^{30}$ The response is controlled by the electromechanical, dielectric, and elastic coefficients, and therefore the polar axis is not expected to be a maximal direction for the piezoelectric coefficient. The phenomenological calculation was done taking into account only the linear intrinsic response. To this the nonlinear intrinsic response should also be added, i.e., the response due to the fact that the piezoelectric coefficient var-

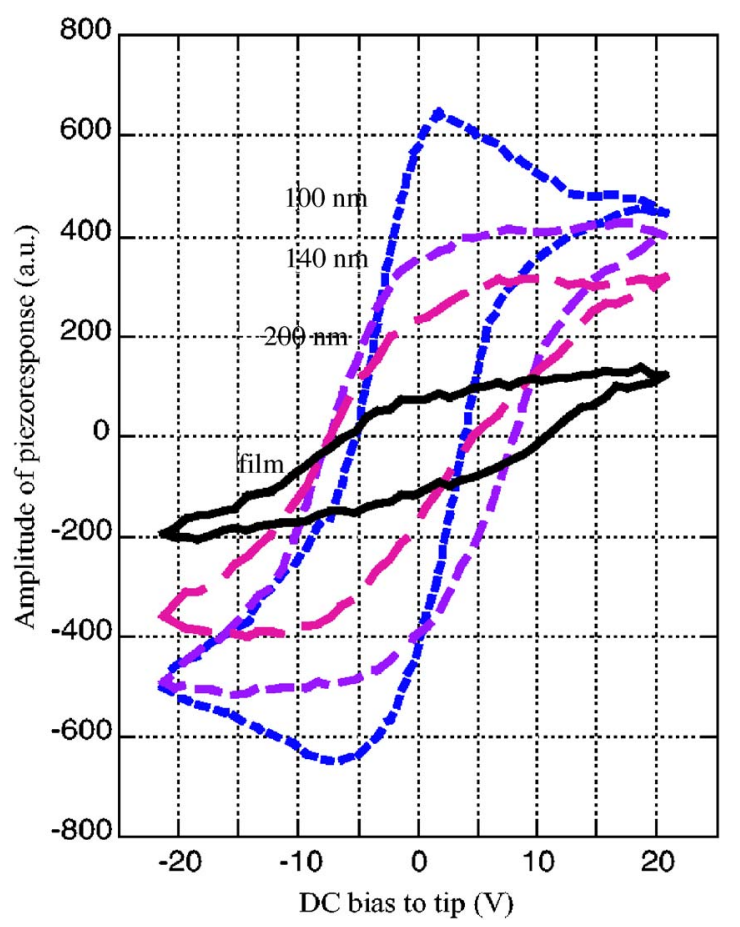

FIG. 1. Amplitude of piezoresponse of PZT patterns with lateral size of 100-200 nm on $\mathrm{SrTiO}_{3}$ substrate (measurement done by PFM technique). (Ref. 28).

ies with applied stress or field. This is significant in thin films where the applied fields or forces are usually high. In addition, the extrinsic response has to be taken into account. The extrinsic response is the combination of all nonlattice effects-domain wall movements, and pinning by the substrate and by defects, defects which are typically substantial in thin films relative to bulk ceramics and single crystals. The understanding of this has developed substantially recently and is discussed in Sec. IV C of the present paper.

To finish the discussion on piezoelectric activity in thin films, it is worth mentioning that all in all the measured magnitude of $e_{31, f}$ of thin films of the appropriate texture and processing conditions is similar in magnitude to that of bulk piezoelectric ceramics (which are of course polycrystalline). This is emphasized in Fig. 2.

Two classes of piezomicrodevices of high interest are cantilever based devices, typified by cantilever switches, and membrane structures as for ultrasonic transducers. These are discussed below.

\section{Microcantilever based piezoelectric components}

The realization of piezoelectric microcantilevers has evolved from demonstrations of single elements, such as PZT coated Si cantilevers for sensing and actuation in AFM heads $^{22}$ to fabrication of microsystems that include arrays of microactuators. One example of an application is that of micromirror arrays for optical scanning. ${ }^{31}$ While the mainstream in micromirror technology is based on electrostatic microactuators, their high voltage operation, nonlinearities, and slow response make the piezoelectric thin film solution attractive. Such was the image projector presented by Daewo Microelectronics at the Asian Display Exhibition on 1998. A 


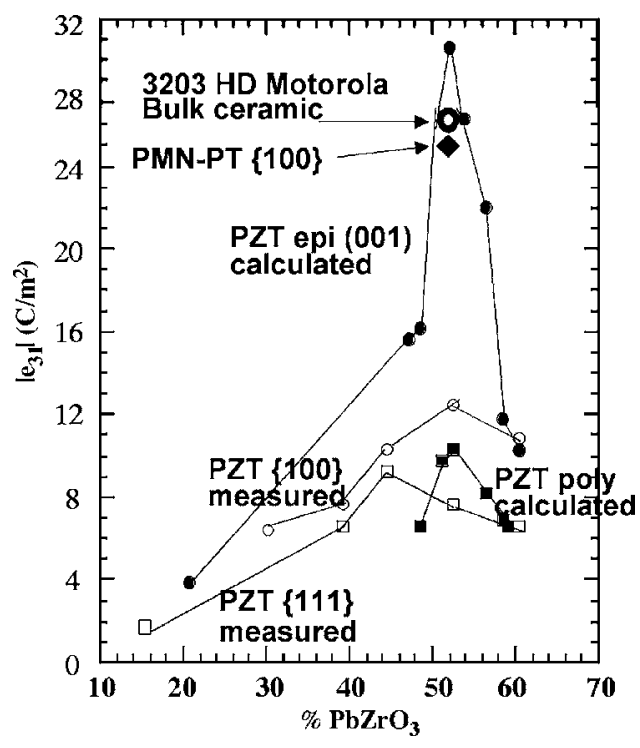

FIG. 2. Transverse piezoelectric response $e_{31, f}$ of PZT films. Measured data are for textured $1 \mu \mathrm{m}$ films on platinized silicon (Ref. 29), whereas calculated data (Ref. 30) are for epitaxial momodomain (001) films. In addition, results from relaxor ferroelectric $\mathrm{Pb}\left(\mathrm{Mg}_{1 / 3} \mathrm{Nb}_{2 / 3}\right) \mathrm{O}_{3}-\mathrm{PbTiO}_{3} 3 \mu \mathrm{m}$ thick textured films and of Motorola bulk state-of-the-art PZT ceramics are inserted (Ref. 445).

chip in this projector had 800000 mirrors that were tilted individually by the activation of the PZT film.

This field is now in full progress and LG Microelectronics reported recently the fabrication of rf MEMS switch arrays containing $90 \times 150 \mu \mathrm{m}^{2}$ size cantilevers for $2.5 \mathrm{~V}$ operation with isolaion $>40 \mathrm{~dB}$ at $2 \mathrm{GHz}^{23,32}$ Another cantilever array reported by the LG group is an array with integrated heaters and piezoelectric sensors that is being studied for microcantilever-type data-storage systems, ${ }^{33}$ Fig. 3. This represents a modification to the IBM Miliped concept ${ }^{34}$ which employs resistive sensing. The piezoelectric version is faster and has lower power consumption.

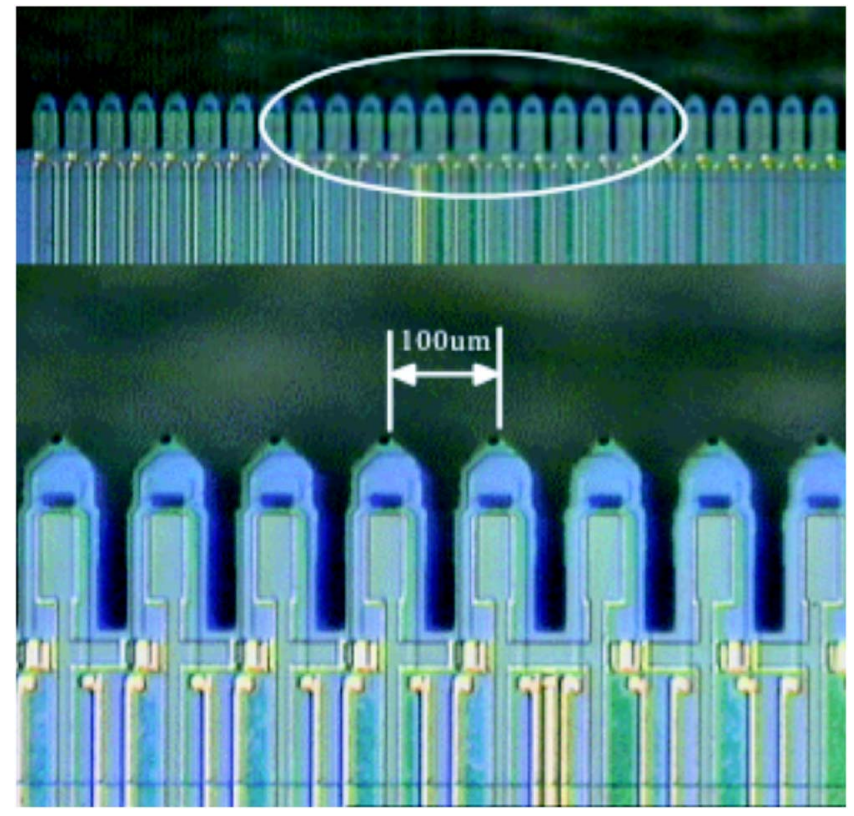

FIG. 3. Partial image of the $100 \times 100$ thermopiezoelectric cantilever array (courtesy of H.-J. Nam, LG Electronics).

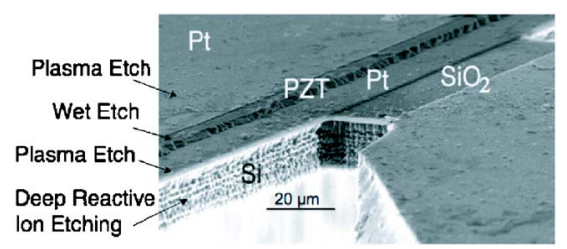

FIG. 4. Process flow for the fabrication of suspended membranes (courtesy of J. Baborowski).

\section{Membrane based micropiezoelectric components}

Like cantilevers, membrane based devices have also evolved from single element demonstrators, e.g., micromotors, to membrane arrays. In particular, ultrasonic applications have attracted much attention recently. ${ }^{35}$ In comparison with bulk piezoelectric ultrasonic arrays, the silicon micromachined ones offer a higher precision and hence a better resolution, the possibility of integration with CMOS, and an easy fabrication of transducers for very high frequencies.

The challenge of making large membranes of uniform thickness was met using semiconductor on insulator (SOI) wafers (Fig. 4). Membranes in the thickness range of $0.5-50 \mu \mathrm{m}$ and tolerances better than $1 \%$ can be fabricated by deep reactive ion etching (DRIE) using the buried oxide as an etch-stop layer. ${ }^{36}$ Since the efficiency of the structure is higher if the membrane is free, there is an effort to produce suspended membranes that are connected to the substrate only by several bridges. This in turn puts severe demands on the fabrication, since the structure becomes sensitive to residual stresses in the membrane. Therefore the stress during the deposition is tailored to compensate stresses due to mismatch with the substrate and the electrodes, and due to the effect of poling. ${ }^{16}$

Several types of membrane based arrays have been demonstrated so far, the following are a few examples. Percin and Khuri-Yakub ${ }^{37}$ have reported a piezoelectric $(\mathrm{ZnO}) \mathrm{mi}^{-}$ cromachined array for liquid injection. They used a bulk piezoelectric element to create the pressure in the liquid, and the piezoelectric thin film $(\mathrm{ZnO})$ on the membrane was used to control the ejection, either by enhancing or stopping the ejection through the orifice of a given element of the array. A short distance presence sensor in air, based on a ring array of micromachined diaphragms coated with PZT, was demonstrated by Yamashita et l. $^{38}$ The advantage relative to conventional ultrasonic sensors is the high directivity.

Piezoelectric micromachined ultrasonic transducers (pMUTs) have lower coupling factors relative to capacitive micromachined ultrasonic transducers (cMUTs). The best value reported for PZT film membranes is $k^{2}=3 \%$ for clamped membranes and 6\% for suspended membranes connected by bridges to the substrate (Fig. 4), while the calculated value is estimated to be in the range of $20 \% .^{35}$ Additional improvement in the piezoelectric coefficient may arise from using better performing compositions (e.g., relaxor ferroelectrics), and by better designs but at present it is not obvious if pMUTs will have an overall advantage over cMUTs. 


\section{B. Polar films in microwave electronics}

With the intensification of use of wireless electronics, it has become essential to produce high frequency systems that are miniaturized, reliable, and of low cost yet high performance. This is particularly true for integration of passive devices since passives are often discrete elements and occupy still nearly $60 \%$ of the total area of handheld devices. Due to their large size, and sometimes the need for special materials, e.g., $\mathrm{LiNbO}_{3}$ or $\mathrm{LiTaO}_{3}$ crystals for surface acoustic wave (SAW) devices, many microwave passive components such as filters and antennas are often placed outside the chip package, requiring inefficient connections and introducing parasitic inductance into the system. Miniaturization and integration of these components onto one substrate can be highly advantageous for cost and size reduction.

Miniaturized tunable capacitors open interesting perspectives for active rf components: miniaturized tunable filters, resonators, phase shifters, antennas, etc., can potentially be manufactured with the desirable characteristics of very high $Q$, narrow bandwidth, low power consumption, low insertion loss, high isolation, and high speed, replacing bulky, multi-component systems. The field of rf MEMSs (we refer to the wide definition of MEMSs as microsystems not necessarily including moving parts) is being intensively developed for this end. The challenge in comparison of other MEMS containing devices is the making of high frequency circuitry that is compatible with MEMS technology. An area that received ample attention recently is that of rf switches. These are either resistive or capacitive switches in which contacts are formed by actuation of a cantilever (usually a metallic cantilever actuated by electrostatic force) either with another metal (resistive switch) or with an insulator which is placed on top of a conductor (capacitive switch). An array of such elements, in which each element is individually addressed, is often used. In parallel to the current development trends in IC technology, MEMS developers also realize that standard materials such as $\mathrm{Si}$, silicon oxide, and silicon nitride do not always provide the most efficient (performance versus cost) and competitive solutions to miniaturization and integration of components. Functional films, such as piezoelectrics, ferroelectrics, magnetic, etc., are therefore being introduced.

Three groups of rf-MEMS components make use of polar thin films: (1) acoustoelectric components based on bulk acoustic waves (BAWs) that control the transmission characteristics of a circuit utilizing the piezoelectric effect, (2) capacitive switches in which the dielectric layer is replaced by a high dielectric constant material are being developed too, to a certain extent (but will not be discussed here), and (3) tunable capacitors in which tunability, namely, the electric field dependence of the permittivity of ferroelectric materials, is used to modify actively the capacitance of the circuit. We summarize here the state of art in polar materials for the first and third groups of components.

\section{Polar ceramics in bulk acoustic wave devices}

A number of filters, resonators, and delay lines operate through the resonance of SAWs that are generated in piezo- electric crystals, in particular, $\mathrm{LiNbO}_{3}$. The resonance frequency is inversely proportional in SAW devices to the distance between the interdigitated electrodes that are placed on the surface of the crystal. The increase in operation frequency of modern communication systems and the interest in miniaturization and integration motivate the replacement of the discrete SAW components by BAW ones based on piezoelectric thin films. In the latter case the transmission frequency $\left(f_{o}\right)$ is related to the thickness $(t)$ of the film and to the acoustic wave velocity in the material $\left(\nu_{s}\right)$ by $f_{o}$ $=\nu_{s} /(2 t)$, placing the thickness, for devices operating at $2-12 \mathrm{GHz}$, in a convenient micron range.

Initial work on BAW thin film resonastors was done by Lakin and Wang ${ }^{39}$ but most development dates less than ten years. BAW resonators are fabricated in three configurations: bulk micromachined film bulk acoustic resonators (FBARs) offering excellent temperature stability; surface micromachined FBARs, offering flexibility of design; and solidly mounted resonators (SMR) in which the suspended structure is replaced by an acoustic mirror built of alternating low and high acoustic impedance layers ${ }^{40}$ thus offering additional robustness.

The material of choice for BAW devices is $\mathrm{AlN},{ }^{41}$ although devices based on other ceramic films, e.g., $\mathrm{ZnO}$, have been demonstrated too. ${ }^{42} \mathrm{AlN}$ is a polar, nonferroelectric material of the wurzite structure. Therefore, in order to be fully active it has to be grown in a perfect [001] orientation and without any inversion of the polarization vector throughout the film. The material exhibits low leakage current, low dielectric constant $\left(\varepsilon_{r}=10.2\right)$, and low dielectric and elastic losses. The piezoelectric coefficients are moderate $\left(d_{33}\right.$ $=5.15 \mathrm{pV} / \mathrm{m}) .{ }^{43}$ Due to its high elastic modulus, its sound velocity is high $(5000-10000 \mathrm{~m} / \mathrm{s}$ ) and its limiting frequency is estimated to be above $100 \mathrm{GHz}^{44}$ Its coupling coefficient $\left(k_{t}^{2}=6.5 \%\right)$ is sufficient to obtain reasonable bandwidth. The coupling coefficient of $\mathrm{ZnO}$ is somewhat higher $\left(k_{t}^{2}=7.5 \%\right)$, but $\mathrm{ZnO}$ is more problematic in processing, and becomes easily lossy. An important advantage of BAW components based on AlN films is the low temperature coefficient of the resonance frequency. Reported values of temperature coefficients are -19 to $-21 \mathrm{ppm} / \mathrm{K}$ for $\mathrm{AlN}$ SMR filters, $0 \mathrm{ppm} / \mathrm{K}$ for compensated AlN thin film bulk acrostic resonators (TFBARs), ${ }^{45}$ while $\mathrm{ZnO}$ SMR filters are reported to exhibit $-31 \mathrm{ppm} / \mathrm{K}$ for $\mathrm{ZnO}^{46}$ This plays in favor of AlN.

As an example, Fig. 5 shows the performance of a BAW $7.9 \mathrm{GHz}$ filter for a microwave link in a telephone network based on an AlN thin film. ${ }^{47}$

One of the attractive features of AlN is the possibility of processing highly performing films at low processing temperature $\left(\approx 200{ }^{\circ} \mathrm{C}\right)$. In this way standard silicon or GaAs substrates can be used as well as bottom electrodes of nonprecious metals, and the resonators can be readily integrated with CMOS. On-wafer packaging techniques can be used to fabricate small BAW filter devices suited for system in package (SiP) solutions. In comparison with SAW components, thin film components are less expensive, since the manufacturing is based on batch IC technology. In addition the thin 

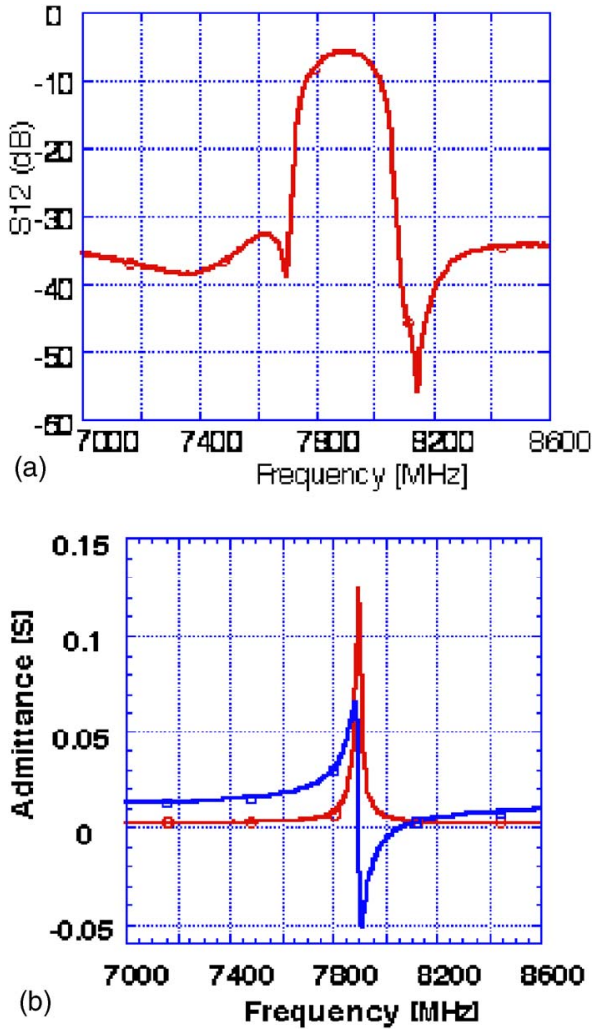

FIG. 5. Performance of two-section $\pi$ filter based on AIN SMR. The measured S12 scattering parameters in (a) give insertion loss of $7 \mathrm{~dB}$ with bandwidth of $200 \mathrm{MHz}$ for central frequency of $7.9 \mathrm{GHz}$. The resonance of a single resonator $\left(30 \mu \mathrm{m}^{2}\right)$ gave coupling coefficient of $5.9 \%$ and $Q$ factor of 460 (b) (Ref. 47).

film devices are smaller in size, having good power handling capabilities, lower temperature drift than the SAWs, and smaller noise due to pyroelectric current.

The commercialization of polar thin film based filters is advancing fast. ${ }^{48}$ Applications for BAW filters include frontend filters for Universal Mobile Telecommunications System (UMTS), Global System for Mobile communication (GSM), bluetooth, and other standards. ${ }^{49}$ The annual market for microwave (MW) filters for handheld devices is estimated in billions of units, and thin film based filters will likely capture a large portion of it.

\section{Ferroelectrics for tunable microwave applications}

$a$. The need for varactors. Motivated by increasing speed and bandwidth requirements, the III-V semiconductor industry has recently made considerable progress in developing transistors with transit time and maximum oscillation frequencies well above $100 \mathrm{GHz}$. The traditional digital silicon industry also, driven by increasing market demands and cost constraints, has put substantial effort in developing analog/ microwave circuits. Silicon based bipolar and CMOS transistors with transit times and maximum oscillation frequencies above $100 \mathrm{GHz}$ are now available, making it possible to develop monolithic microwave ICs (MMICs) for frequencies up to $50 \mathrm{GHz}$. Along with the transistors, varactors are the other widely used components used in microwave technology for analog (nondigital) tuning purposes. In spite of the progress achieved in the transistor technology, no semicon- ductor varactors with high enough $Q$ factor and tunability are available for frequencies above 10-20 GHz. Typically, the $Q$ factors of semiconductor varactors decrease with the increased frequency as $Q \sim f^{-1}$, and at $50 \mathrm{GHz}$ it is of the order 10 or less. There is a lack of varactors with adequate (compatible with the transistors) performance in this frequency range.

The current progress indicates that, to a certain degree, the "varactor gap" may be filled in by ferroelectric varactors. After several decades of research considerable progress has been achieved in tunable permittivity ferroelectrics, making it possible to develop ferroelectric varactors with performances better than semiconductor analogs in the frequency range above $10-20 \mathrm{GHz}^{50,51}$ Thin film ferroelectric varactors have a substantially higher $Q$ factor at microwave and millimeter wave frequencies, higher tuning speed, and lower drive power consumption. Ferroelectric varactors, and tunable devices based on them, have the potential for easy integration with standard $\mathrm{Si}$ and GaAs processes. Additionally, due to the high dielectric permittivity, the sizes of tunable components based on ferroelectrics may be smaller in comparison with other technologies. Extra flexibility in terms of tuning, enhanced functionalities, and performances of tunable devices may be achieved by combination of ferroelectrics with ferrites, ${ }^{52,53}$ ferroelectrics with semiconductors, ${ }^{54}$ or ferrites with semiconductors. ${ }^{55}$ Devices based on such multifunctional materials offer dual, i.e., electric, magnetic tuning possibility, and extra flexibility in designing and shaping the device performances. For example, in delay lines it is possible to tune the delay time while maintaining the input/ output impedances at a desired level.

b. Ferroelectric varactors and devices. Both ferroelectric (polar) and paraelectric phases may be useful in tunable microwave devices. ${ }^{56}$ However, the paraelectric phase is often preferred since it has no hysteresis associated with the domain walls. In this respect the quantum paraelectrics $\left[\mathrm{SrTiO}_{3}, \mathrm{KTaO}_{3}\right.$, and $\mathrm{CaTiO}_{3}$ (Refs. 57 and 58)] have been given priority for some time, although they need to be cooled down to cryogenic temperatures in order to get reasonable tunabilities. On the other hand in thin film devices based on these materials, and especially in the solid solutions of $\mathrm{Ba}_{x} \mathrm{Sr}_{1-x} \mathrm{TiO}_{3}$ (BST), high electric fields and reasonable tunability may be achieved at room temperature at relatively low dc voltages. For applications in industrial devices $\mathrm{Ba}_{x} \mathrm{Sr}_{1-x} \mathrm{TiO}_{3}$ seems to be the material of choice. It is the most studied material at microwave frequencies, which allows microwave engineers to use the available data without getting too much involved in a materials study. In addition, it allows control of the Curie temperature by simply changing the $\mathrm{Ba}$ content.

The performance of ferroelectric devices depends not only on the composition of the film but also on the strain, defects, electrode/ferroelectric interface chemistry, fabrication method, design, etc. Ferroelectric varactors, and devices based on them, may have parallel-plate or coplanar-plate designs, Fig. 6. The former offers smaller tuning voltages, while the latter offers the possibility of trading tunability for lower losses. 


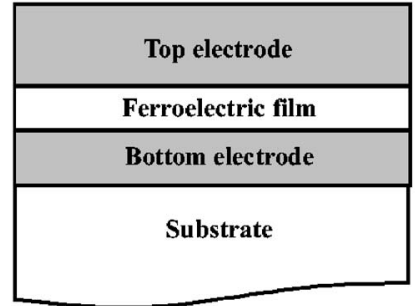

(a)

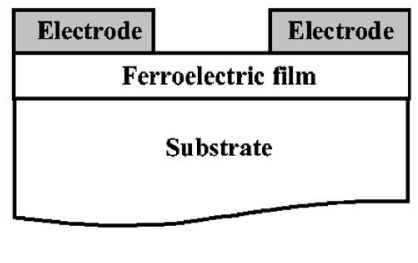

(b)
FIG. 6. Parallel-plate (a) and coplanar-plate (b) ferroelectric varactors.

Typical frequency dependencies of the capacitance and $Q$ factor of a parallel-plate ferroelectric varactor are shown in Fig. 7. In this case the varactor is fabricated on a high resistivity $(5 \mathrm{k} \Omega \mathrm{cm})$ silicon substrate with adhesive $\mathrm{TiO}_{2}$ $(15 \mathrm{~nm})$ and $\mathrm{Pt}(100 \mathrm{~nm})$ layers. The $\mathrm{Ba}_{0.25} \mathrm{Sr}_{0.75} \mathrm{TiO}_{3}$ film is $300 \mathrm{~nm}$ thick. The bottom and top electrodes are made of a $0.5 \mu \mathrm{m}$ thick Au film and $50 \mathrm{~nm}$ Pt layer. The diameter of the top plate is $10 \mu \mathrm{m}$. For comparison, in Fig. 7(b), the performance of varactor based on $\mathrm{Pt}(200 \mathrm{~nm}) / \mathrm{TiO}_{2} / \mathrm{SiO}_{2} / \mathrm{Si}$ structure (i.e., thinner bottom electrode) is also shown. The use of thicker Au bottom electrode substantially increases the $Q$ factor of the varactor. The capacitance and the tenability are fairly frequency independent. The tunability, $T(V)$ $=[C(0)-C(V)] / C(0)$, of a BST varactor at $25 \mathrm{~V}, T_{c}(25)$, is more than $40 \%$ in the frequency range of $1 \mathrm{MHz}-45 \mathrm{GHz}$. In the frequency range of $5-40 \mathrm{GHz}$ the $Q$ factor of BST varactors fabricated on thick bottom $\mathrm{Au} / \mathrm{Pt}$ electrode is more than 40 , which is competetive with the best semiconductor analogs, Fig. 7(b). ${ }^{51}$ Additionally, ferroelectric varactors have symmetric $C-V$ characteristics, as do heterojunction barrier varactors $(\mathrm{HBVs}){ }^{59}$
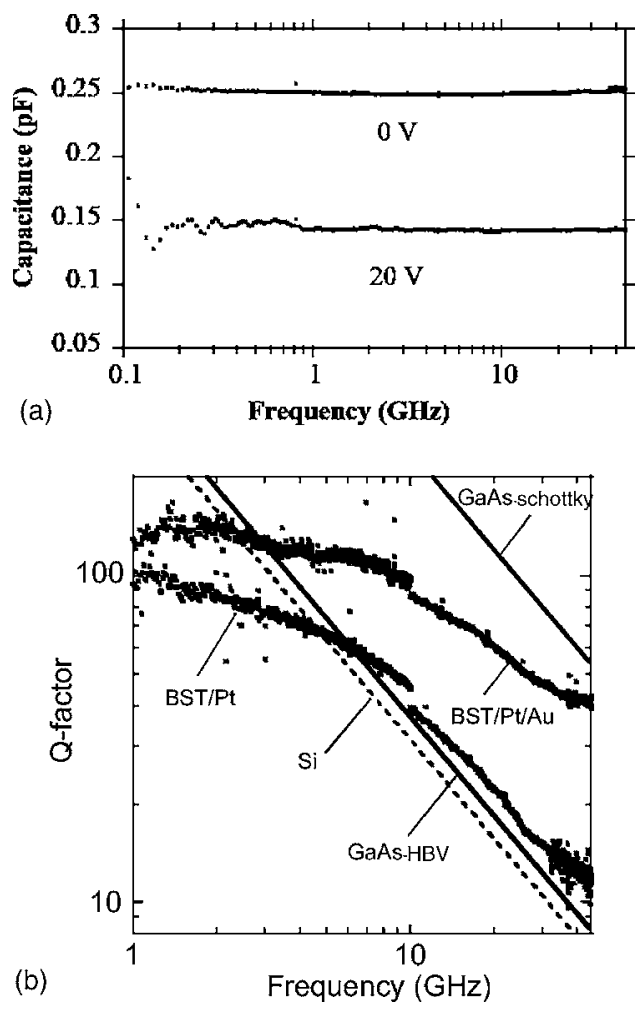

FIG. 7. Capacitance (a) and $Q$ factor (b) of varactors vs frequency.
The frequency independent tunability (up to $50 \%$ or more), high tuning speed ( $<1.0 \mathrm{~ns}$ ), extremely small leakage currents and dc control power, high breakdown field, and radiation hardness are the main advantages of ferroelectric varactors. Recently substantial progress has been achieved in commercialization of tunable microwave components based on ferroelectrics. ${ }^{60,61}$ In fact, ferroelectrics are gaining ground for tunable microwave applications, including lumped element (device sizes much smaller than the microwave wavelength) and distributed element devices, such as varactors, phase shifters, delay lines, tunable filters, antennas, etc. ${ }^{50,56,60,61}$ Nevertheless, problems such as hysteresis, temperature dependence of parameters (such as permittivity of the ferroelectric material), etc., still need adequate treatment. One of the fundamental problems concerning the practical use of tunable ferroelectric devices is considered below. It has to do with the strong temperature dependence of the parameters of tunable ferroelectric devices associated with the inherent temperature dependence of the ferroelectric's permittivity.

c. Reduction of the temperature dependence. A couple of methods for reducing the temperature dependencies have been discussed in the past, including the use of Peltier elements, or electronic feedback (on circuit application level) to control the drive dc voltage. Temperature stabilization may also be achieved physically, by using composite ferroelectric layers. In this case two or more layers of ferroelectrics with different Curie temperatures are used so that the temperature interval of interest, i.e., the temperature range of the device operation, is between the temperatures corresponding to the peaks of the permittivity of the layers. ${ }^{62}$ The former methods are not cost effective. The latter method suggests that one of the ferroelectric layers operates in a polar phase, which may cause hysteresis in $C-V$ dependences. In general, in tunable microwave applications, hysteresis is an unwanted effect. It causes ambiguity in the bias/derive voltages required for reaching a certain value of the capacitance (state of the device).

A simple and cost effective way of temperature stabilization is shown in Fig. 8(a).$^{63}$ The bias network consists of a capacitive voltage divider and a dc decoupling network (shown is a simple low pass filter based on an inductor). Ferroelectric varactors and microwave devices based on them have extremely low leakage currents. Hence, to bias (control/drive) them, a simple capacitive voltage divider may be used, as shown in Fig. 8(a). The voltage divider consists of a fixed capacitor, $C$, based on a conventional dielectric, preferably with good temperature stability, in series with a temperature sensitive capacitor, $C_{T}$, preferably made of the same or similar ferroelectric as the one used in the varactor. At given bias voltage $V_{d c}$, the voltages dropped across capacitors $C$ and $C_{T}$ are

$$
V_{C}=\frac{C_{T}}{C+C_{T}} V_{\mathrm{dc}}
$$


(a)

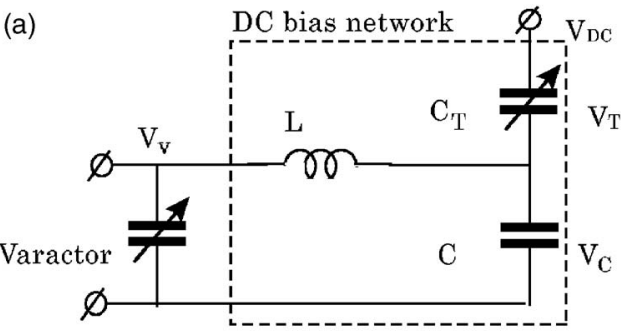

(b)

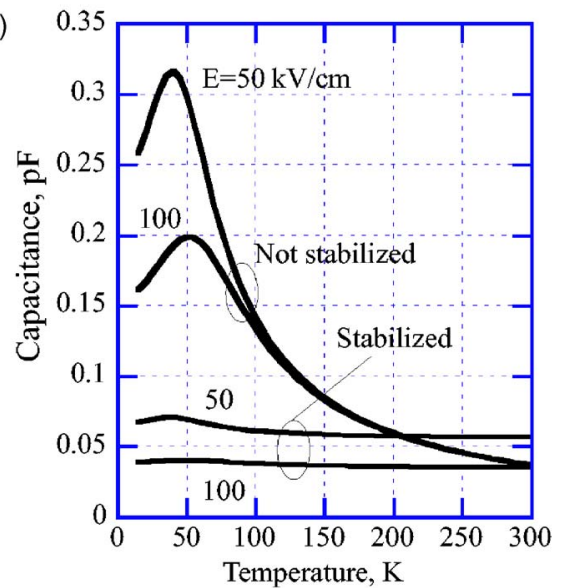

FIG. 8. The concept of temperature stabilization (a), and the temperature dependences of nonstabilized and stabilized $\mathrm{SrTiO}_{3}$ varactors under dc fields of $50 \mathrm{kV} / \mathrm{cm}$ and $100 \mathrm{kV} / \mathrm{s}(\mathrm{b})$.

$$
V_{T}=\frac{C}{C+C_{T}} V_{\mathrm{dc}} .
$$

The capacitor $C_{T}$ included in the voltage divider, Fig. 8(a), is characterized by a temperature dependence similar to the controlled varactor itself. Due to this dependence a voltage drop redistribution takes place in the capacitive voltage divider when the temperature changes. The voltage across $C_{T}$ decreases when its capacitance increases with the changing temperature. As follows from Eqs. (3) and (4), the voltage drop across capacitor $C$ is $V_{C}=V_{d c}-V_{T}$. For a lossless decoupling network [i.e., inductor in Fig. 8(a)] the voltage across the varactor is the same as the voltage $V_{C}, V_{V}=V_{C}$. Hence a temperature-dependent reduction in the capacitance of the varactor causes a corresponding capacitance reduction in capacitance $C_{T}$, leading to a reduced voltage $V_{V}$ applied to the varactor. As a result the reduction of varactor capacitance caused by temperature is compensated by reduced voltage $V_{V}$. In a similar way, any capacitance increase of the varactor due to temperature change will be compensated by an increased voltage $V_{V}$. The original, i.e., nonstabilized, and the temperature stabilized performance of a ferroelectric varactor are shown in Fig. 8(b). In this example the temperature and field dependent permittivity model of $\mathrm{SrTiO}_{3}$ (Ref. 64) is used. The minimum value of the capacitance $C_{T}$ and, hence, the minimum required bias voltage $V_{d c}$ is selected from the condition

$$
\Delta C_{V}=\Delta C_{T},
$$

where $\Delta C_{V}$ is the voltage induced change in capacitance required to compensate the temperature induced changes $\Delta C_{T}$.

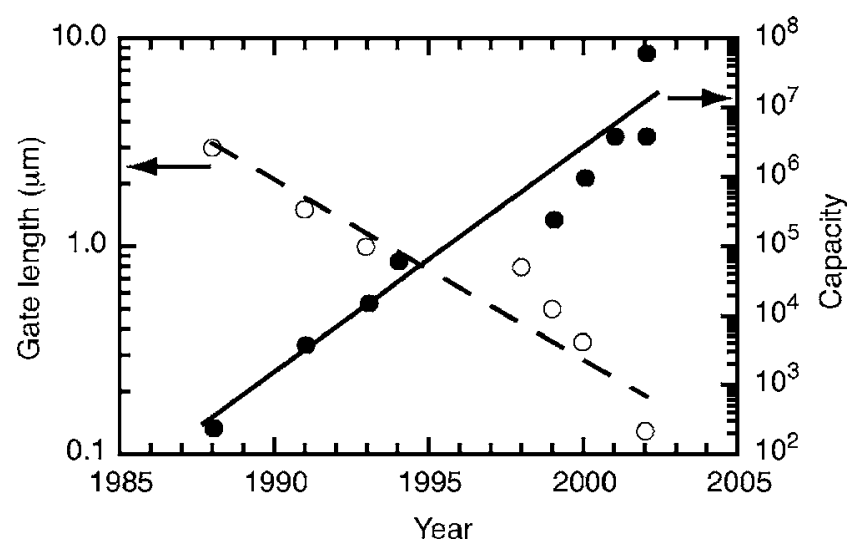

FIG. 9. Progression of FRAM minimum transistor gate length and memory capacity since the demonstration of integrated nonvolatile ferroelectric memory.

The proposed method has some disadvantages. First of all, it works only when the devices are biased. No temperature stabilization is available for zero bias devices. Additionally, the required tuning drive/voltages are somehow larger. This is the price one has to pay for the simplicity of the temperature stabilization. It may be acceptable in many applications.

\section{Ferroelectric thin films in FRAM}

The principle of nonvolatile ferroelectric random access memories (FRAMs) is based on the polarization reversal by an external applied electric field of metal-ferroelectric-metal capacitors: ${ }^{11,65,66}$ The computational " 0 " and " 1 " are represented by the nonvolatile storage of the negative or positive remanent polarization state, respectively. The destructive readout operation is performed via the displacement current. With respect to the electronic properties, FRAMs show attractive features, for example, write and read cycle times in the sub-100 ns range and a low power consumption, which are in most cases superior to the performance of other nonvolatile technologies.

Ferroelectric memory integrated with silicon CMOS was demonstrated in 1987 . $^{6}$ The initial ferroelectric memory test chip consisted of only 256 bits; each bit contained two $\mathrm{PbZr}_{0.5} \mathrm{Ti}_{0.5} \mathrm{O}_{3}$ (PZT) capacitors that formed a nonvolatile shadow RAM for the accompanying six-transistor static random access memory cell (6T SRAM). Despite the low density and the large cell size, demonstration of an integrated ferroelectric-based nonvolatile memory provided impetus for the development of higher density test chips and ferroelectric random access memory products. ${ }^{6,67,68}$ Mass production of FRAM products commenced in 1992 after the development of a 4 kbyte memory that utilized a high reliability two transistor, two-capacitor (2T2C) cell that was more area efficient than the early shadow RAM cells. ${ }^{6,67}$ Since 1992 , there has been an exponential growth in FRAM capacity to a current maximum of 64 Mbytes and an exponential decrease in the integrated CMOS transistor gate length to $0.13 \mu \mathrm{m}$, as shown in Fig. 9.

Although PZT was the material of choice for demonstration of integrated ferroelectric memories, difficulties with 
achieving greater than $10^{12} \mathrm{read} /$ write cycles led some researchers to investigate other ferroelectric thin film materials. ${ }^{10}$ Notable alternatives to PZT that were investigated include $\mathrm{SrBi}_{2} \mathrm{Ta}_{2} \mathrm{O}_{9} \quad(\mathrm{SBT}){ }^{69,70}(\mathrm{BiLa})_{4} \mathrm{Ti}_{3} \mathrm{O}_{12},{ }^{71}$ $\mathrm{BaMgF}_{4}, \mathrm{Bi}_{4} \mathrm{Ti}_{3} \mathrm{O}_{12},{ }^{72}$ and $\mathrm{Pb}_{5} \mathrm{Ge}_{3} \mathrm{O}_{11}{ }^{73}$ The objective was to find a ferroelectric material that showed little or no degradation of the switchable ferroelectric polarization while cycling the bit capacitor structure through typical read and write operations. Of the alternative ferroelectric materials, the Bi-based compounds exhibited the most improved read/ write cycling endurance, but only SBT has led to marketable FRAM products. The SBT-based products are currently limited to low memory capacity (less than 16 kbytes) smartcard applications, although test chips with capacity as high as 4 Mbytes have been demonstrated. ${ }^{74}$

A second approach for circumventing the limitations to $\mathrm{read} /$ write endurance was to employ a nondestructive readout of the nonvolatile ferroelectric capacitor polarization state. ${ }^{72}$ Initial FRAM designs had relied on a destructive readout (DRO) scheme where the polarization state of the memory bit had to be switched in order to sense the stored memory state. Once the bit was read, the memory state was rewritten into the ferroelectric capacitor by resetting the polarization. Switching the polarization during both write and read operations caused reduced endurance. Since many memory applications require more read accesses than writes, a nondestructive readout (NDRO) of the ferroelectric capacitor would necessarily increase endurance. Several NDRO ferroelectric memory designs have been investigated, but none have led to viable products to date. All proposed NDRO ferroelectric memories seem to suffer from a common data disturb problem arising from partial back switching of the remanent polarization state during each read cycle, which leads to long term degradation of the read signal margin, i.e., loss of data state retention. ${ }^{75}$

Since the demonstration of FRAM, focus on PZT capacitor process development and DRO design for reliability have lead to FRAM products with unlimited read/write endurance and $10 \mathrm{yr}$ data retention over industrial operating temperature specification of -40 to $85{ }^{\circ} \mathrm{C}{ }^{76,77}$ Some FRAM products have now achieved $45 \mathrm{yr}$ retention and qualify for automotive applications. Many commercial FRAM products utilize a $2 \mathrm{~T} 2 \mathrm{C}$ cell with a $0.5 \mu \mathrm{m}$ minimum CMOS gate length. ${ }^{78}$ In these devices, the ferroelectric capacitors are formed on top of a field oxide such that the capacitors are next to the accompanying transistors and each component of the cell occupies a uniquely defined area. This architecture is referred to as the capacitor-over-field-oxide (COFO) architecture and it results in a relatively large $60 F^{2}(F$ is the metal half-pitch) cell size for the $2 \mathrm{~T} 2 \mathrm{C}$ devices. Each capacitor in the cell has an associated transistor that controls current flow to each ferroelectric capacitor [Fig. 10(b)]. The capacitors always have opposite polarities, therefore, one capacitor will produce a switching signal while the second capacitor will produce a nonswitching signal. A 1 state is read if the switching signal is detected on the bit line and a 0 state is read if the switching signal is detected on the bit-line complement. Because the $2 \mathrm{~T} 2 \mathrm{C}$ cell occupies a large area, it is only used for FRAMs with densities of $256 \mathrm{kbits}$ or less.

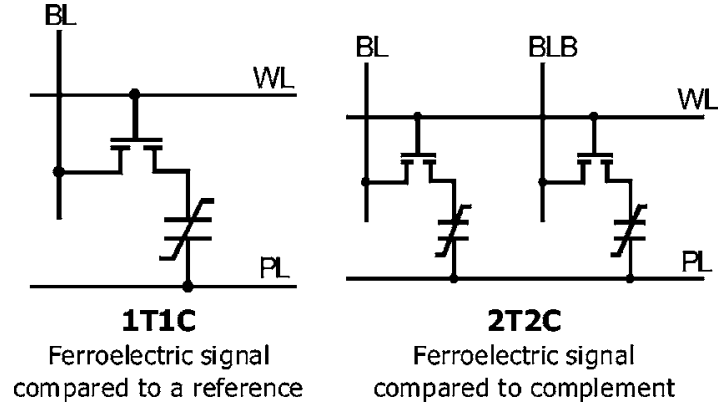

FIG. 10. Schematic diagrams of 1T1C (left) and 2T2C (right) FRAM bit cells.

A significantly smaller cell size of $33 F^{2}$ is achieved by eliminating the complementary capacitor and transistor in each cell and accessing a reference signal from a capacitor placed outside the cell array. ${ }^{79}$ If a switching charge pulse is detected from the capacitor of the one-transistor, onecapacitor (1T1C) cell [Fig. 10(a)], the cell capacitor is said to be in the 1 state, whereas if no switching charge is detected, the cell capacitor is in the 0 state. A cross section of a 1T1C cell with the COFO architecture built on a $0.35 \mu \mathrm{m}$ CMOS process is shown in Fig. 11. Connection of the Al M2 bitline to the capacitor $\mathrm{IrO}_{2}$ top electrode is gated by the adjacent transistor, while $\mathrm{Al} \mathrm{M} 1$ and the $\mathrm{W}$ plugs complete the conduction path. The ferroelectric layer below the top electrode consists of greater than $90 \%\{111\}$ crystallographically textured $\mathrm{PbZr}_{0.4} \mathrm{Ti}_{0.6} \mathrm{O}_{3}$ doped with $\mathrm{La}, \mathrm{Ca}$, and $\mathrm{Sr}^{80}$ High reproducibility of the $\{111\}$ texture is achieved by sputtering the PZT onto $\{111\}$ textured Pt, which acts as a template during crystallization of the PZT perovskite phase. Products with memory capacities up to 1 Mbyte and value added memories with additional features such as a real-time clock and system supervisor are now manufactured with the 1T1C FRAM cell and the $0.35 \mu \mathrm{m}$ process. Applications for the 0.5 and $0.35 \mu \mathrm{m}$ FRAM devices include electrical power meters, printer configuration memories, data loggers, video games, and toy watches.

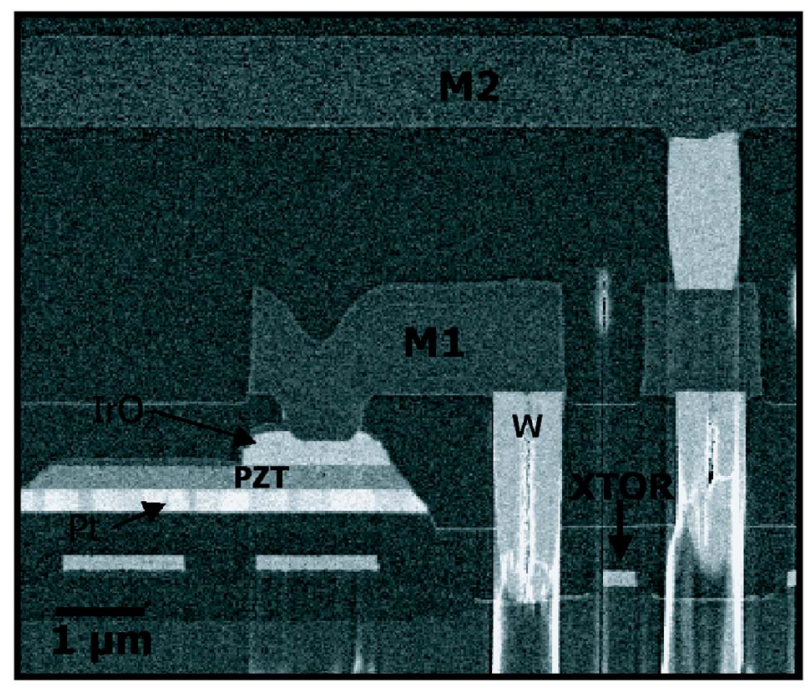

FIG. 11. Scanning electron microscope cross-section image of a 1T1C COFO FRAM cell for 256 kbyte memory manufactured on a $0.35 \mu \mathrm{m}$ three-level-Al CMOS process. 


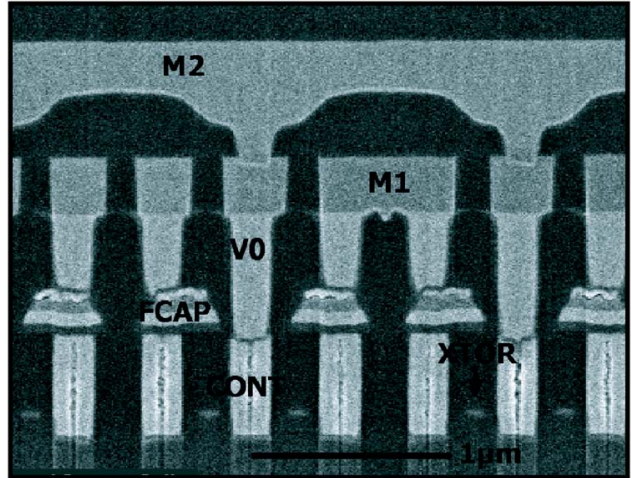

FIG. 12. Scanning electron microscope cross-section image of several 1T1C COP FRAM cells for a 4 Mbyte memory manufactured on a $0.13 \mu \mathrm{m}$ fivelevel-Cu CMOS process.

Because of the success of FRAM mass production at the 0.5 and $0.35 \mu \mathrm{m}$ process nodes and the potential for a variety of stand-alone and embedded applications, several manufacturers have focused development on continued scaling of FRAM to achieve higher capacity and higher density. ${ }^{81-85}$ As part of the scaling effort, the FRAM cell has undergone an additional change in architecture to reduce cell size. A cell factor decrease is achieved by building the ferroelectric capacitor on top of a plug that contacts an underlying access transistor, as shown in Fig. 12. For the capacitor-on-plug (COP) architecture shown in Fig. 12, a cell size of $18 F^{2}$ was achieved and it was used to demonstrate 4 and 64 Mbyte memories. ${ }^{86,87}$

The COP architecture introduces demands on the ferroelectric capacitor materials since the capacitor is grown on a conducting plug material (as apposed to field oxide) that must be protected from oxidation to ensure low contact resistance. The use of a TiAlN barrier between a W plug and an Ir bottom electrode provides the necessary interdiffusion and oxidation resistance needed for growth of $\mathrm{PbZr}_{0.2} \mathrm{Ti}_{0.8} \mathrm{O}_{3}$ by metal-organic chemical vapor deposition (MOCVD). A mixture of (001) and (100) PZT grains results from the MOCVD process and it is found that films with $(001) /(100)$ ratios of greater than $50 \%$ provide for sufficient switchable polarization and reliability for COP FRAMs. ${ }^{88}$ The bit capacitor is completed with an $\mathrm{IrO}_{2} / \mathrm{Ir}$ top electrode and TiAlN hard mask that provides for single mask etching of the capacitor stack. After the bit capacitor is formed it is coated with an $\mathrm{AlO}_{x}$ barrier layer that protects against degradation of the ferroelectric switching during subsequent interlevel dielectric, $\mathrm{W}$ plug, and $\mathrm{Cu}$ metal interconnect deposition and etch. Ultimately, the materials modifications to the COP ferroelectric capacitor module residing between the contact and metal-1 layers allow for integration with a five-level $\mathrm{Cu}$ metallization backend with the addition of only two masks to the standard $0.13 \mu \mathrm{m}$ logic process.

In addition to the high cell density provided by the COP architecture, the five-level $\mathrm{Cu}$ process allows for designs with improved array efficiency and access times. Figure 13 illustrates the decrease in access time and increase in density as a function of parallel FRAM product and test chip memory capacity ranging from 64 kbytes to 64 Mbytes. Access times as fast as $30 \mathrm{~ns}$ and densities as high as

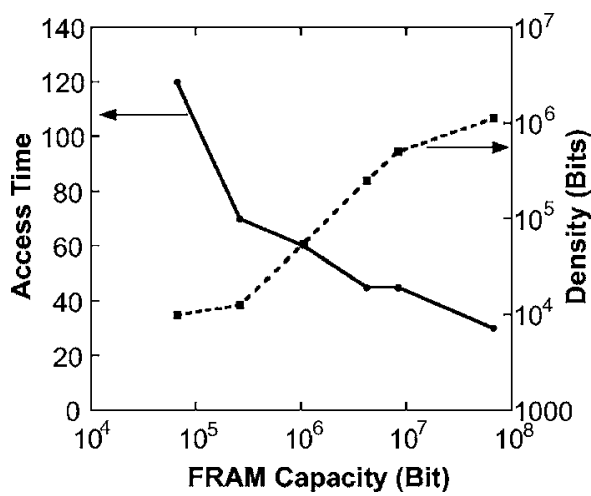

FIG. 13. Change in access time and bit density for increasing memory capacity of FRAM products and test chips.

$1 \mathrm{Mbyte} / \mathrm{mm}^{2}$ make FRAMs interesting as a replacement in the battery-backed SRAM market as well as for embedded applications such as can be found in cell phones.

A comparison of several of the critical physical parameters for ferroelectric capacitors and 1T1C memory products is given in Table I for the $0.5,0.35$, and $0.13 \mu \mathrm{m}$ FRAM generations. Memory capacity has been increased by changing the cell architecture and decreasing the cell size by more than a factor of 20 . In order to achieve the reduction in cell size, the ferroelectric capacitor area also had to be decreased by approximately a factor of 7 . In addition, the ferroelectric thickness was decreased to accommodate the lower core voltage need to be compatible with the supporting CMOS transistors. The scaling changes were made while maintaining $10 \mathrm{yr}$ data retention and unlimited $\left(>10^{15}\right) \mathrm{read} / \mathrm{write}$ endurance cycles. From this table, the challenges for future FRAM scaling can be deduced: the capacitor size must be reduced to fit within the required cell area while maintaining sufficient reproducibility and signal margin for sense amplifier differentiation between a zero and one data state, the ferroelectric thickness must be scaled to allow switching in voltage ranges compatible with the supporting CMOS transistors, retention and cycling enduring must be maintained,

TABLE I. Comparison of 1T1C FRAM capacity, architecture, and critical parameters produced at the $0.5,0.35$, and $0.13 \mu \mathrm{m}$ technology nodes.

\begin{tabular}{|c|c|c|c|}
\hline & $0.5 \mu \mathrm{m}$ & $0.35 \mu \mathrm{m}$ & $0.13 \mu \mathrm{m}$ \\
\hline Density & 4-256 kbytes & 4 kbytes -1 Mbyte & 4-64 Mbytes \\
\hline Architecture & $\mathrm{COFO}$ & $\mathrm{COFO}$ & $\mathrm{COP}$ \\
\hline $\begin{array}{l}\text { Metallization type/ } \\
\text { No. of layers }\end{array}$ & Aluminum/2 & Aluminum $/ 3$ & Copper/5 \\
\hline $\begin{array}{l}\text { Cell factor (metal } \\
1 / 2 \text { pitch base) }\end{array}$ & 35 & 33 & $23-18$ \\
\hline Cell size $\left(\mu \mathrm{m}^{2}\right)$ & 15.8 & 8.3 & $0.71-0.54$ \\
\hline Core voltage (V) & $2.7-5.5$ & $2.7-3.8$ & 1.5 \\
\hline Capacitor area $\left(\mu \mathrm{m}^{2}\right)$ & 3.0 & 2.0 & $0.45-0.25$ \\
\hline Psw $\left(\mu \mathrm{C} / \mathrm{cm}^{2}\right)$ & 30 & 30 & 40 \\
\hline PZT thickness (nm) & 200 & 150 & 70 \\
\hline Data retention $(\mathrm{yr})$ & $>10$ & $>10$ & $>10$ \\
\hline $\begin{array}{l}\text { Read/write endur. } \\
\text { (No. of cycles) }\end{array}$ & $10^{10} /$ unlim. & Unlim. & Unlim. \\
\hline Access time (ns) & $120-70$ & 55 & $45-15$ \\
\hline Active current (mA) & $15-25$ & $<20$ & $<20$ \\
\hline
\end{tabular}


and addition of the ferroelectric capacitor module must be compatible with the CMOS process with which it will be integrated.

\section{Alternative memories based on ferroelectric materials}

FRAMs are an essential and well developed technology and their application in products is the most evident prove for a reliable technology. On the other hand, there are a number of alternative approaches for nonvolatile storage on the basis of ferroelectric materials. The following section discusses three possibilities: ferroelectric field effect transistors (FeFETs), ferroresistive based storage devices, and scanning probe related methods including there individual development status.

\section{Ferroelectric field effect transistors (FeFETs)}

A $1 \mathrm{~T}$ cell (FeFET) is in principle a metal-oxidesemiconductor FET (MOSFET) whose gate dielectric is ferroelectric. An advantage of this cell type is that the read operation is nondestructive but the main disadvantage is the fact that the current achievable retention time is very short for use in a nonvolatile memory. ${ }^{89-91}$ The idea of the FeFET was presented in a patent in $1957 .{ }^{92}$ Further milestones include its realization by Moll and Tarui in $1963,{ }^{93}$ with triglycine sulphate (TGS) on a CdS substrate as well as the approach for implementing FeFET on silicon by $\mathrm{Wu}$ in $1974 .^{94}$ Although there has been tremendous research on FeFETs with various material combinations and different layouts, the FeFET has not been able to become product. Nonetheless, the brilliant concept of the FeFET continues to attract much interest. ${ }^{11,89}$ Important pros and cons of the FeFET are discussed in the following.

We wish to point out that CMOS integration issues (i.e., the compatibility of Si technology in combination with complex oxides) are somewhat different in the case of FRAM and FeFET. In a FRAM capacitor over bit-line (COB) cell the ferroelectric is approximately $100 \mathrm{~nm}$ or more apart from the MOSFET. Both devices act as physically independent elements. In an ideal FeFET the ferroelectric is in direct contact with the drain-source channel of the transistor, as shown in Fig. 14(a). The ferroelectric is an active part of the transistor and the FeFET is a single device. The performance of the FeFET is therefore inextricably connected to its interface physics and the electronic properties of this interface. The interface is one of the most serious problems for the FeFET. The transistor properties such as threshold voltage, saturation voltage, or the $C$ - $V$ curve of the gate stack are strongly influenced by localized states (e.g., dangling bonds) and impurities at the interface. Interdiffusion between the ferroelectric and $\mathrm{Si}$ is another bottleneck for the FeFET. Even for the native oxide $\mathrm{SiO}_{2}$ (and for high- $k$ dielectrics) it took years to overcome all the problems in achieving high performance MOSFETs. Essential challenges for the FeFET are the improvement of the retention time and the suppression of serious parasitic effects such as the charge traps at the $\mathrm{Si}$-ferroelectric interface. One possible solution in the case of a FeFET is the incorporation of one insulating buffer layer
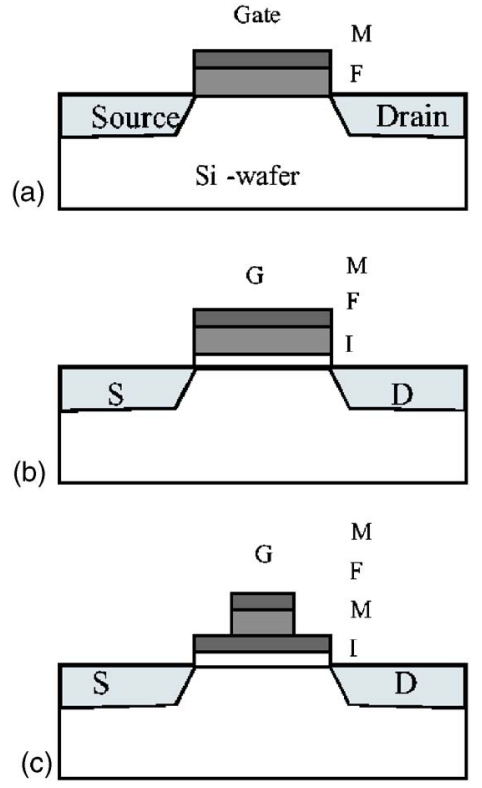

FIG. 14. Schematic cross-sectional views of FeFETs with (a) a MFS, (b) a MFIS, and (c) a MFMFIS gate layer sequence. Please note the area difference between the MFM and MIS structures in (c). M: metal, F: ferroelectric, I: insulator, and $\mathrm{S}$ : semiconductor.

between the $\mathrm{Si}$ and the ferroelectric as schematically shown in Fig. 14(b) to obtain a metal-ferroelectric-insulator-silicon (MFIS) structure. ${ }^{89,90}$ Several buffer layers, for example, $\mathrm{SiO}_{2}, \mathrm{CeO}_{x}$, and $\mathrm{Si}_{3} \mathrm{~N}_{4}$, have been investigated. The most recent results by using alternative gate oxides such as $\mathrm{HfO}_{2}$ or HfAlO show encouraging results. The retention time for the gate layer sequence $\mathrm{Pt} / \mathrm{SBT} / \mathrm{HfO}_{2} / \mathrm{Si}$ was 30 days, the best value observed up to now. ${ }^{89,95}$

Beside a low density of interface states the band offset between $\mathrm{Si}$ and the ferroelectric or the buffer needs to be sufficiently large to avoid electron injection during programming. Although a quasiperfect epitaxial growth of the high- $k$ $\mathrm{SrTiO}_{3}$ on $\mathrm{Si}$ is possible by advanced molecular beam epitaxy (MBE), the small band offset of $\mathrm{SrTiO}_{3}$ on $\mathrm{Si}$ leads to strong current injection and a compensation of the ferroelectric bound charge by electrons. ${ }^{96-98}$ The consequence is again a low retention time. Even more complex gate sequences have been investigated in the form of metal-ferroelectricmetal-insulator-silicon (MFMIS) structures [see Fig. 14(c)]. ${ }^{89,91}$ The technological advantage here is that in this case optimized MFM fabrication from FRAMs is used. Moreover this gate layer sequence allows engineering of the area ratio to optimize the charge matching between the buffer and the ferroelectric. A smaller area of the MFM in comparison to the underlying IMS structure results in lower field strength of the buffer. The maximum charge density for $\mathrm{SiO}_{2}$ is $3.5 \mu \mathrm{C} / \mathrm{cm}^{2}$ and corresponds to an electric field strength of $10 \mathrm{MV} / \mathrm{cm}$-nearly the breakdown field of $\mathrm{SiO}_{2}$. Therefore the material requirements for the ferroelectric materials in FeFETs are somewhat different to those used in conventional FRAMs. To control the source-drain current of a field effect transistor a simulation showed that materials with a low $P_{r}\left(<2 \mu \mathrm{C} / \mathrm{cm}^{2}\right)$ are optimal. Since typical complex oxide ferroelectrics exhibit more than ten times higher $P_{r}$ 


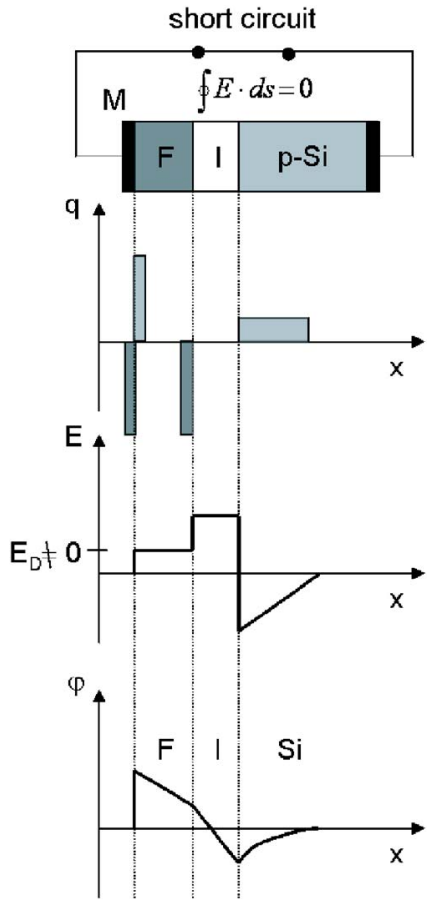

FIG. 15. Layout of a MFIS diode and the charge distribution, the electrical field inside the layers, and the potential across the structure. An ionic charge compensation in the buffer insulator (I) is neglected.

often subloops are used to obtain sufficiently low $P_{r}$ values and in addition, as discussed above, to avoid electric breakdown of the buffer. ${ }^{89}$

The problem of short retention times in FeFETs is illustrated in Fig. 15. The finite depolarization field due to the a noncomplete charge compensation at the semiconductor side of the ferroelectric may lead to the formation of $180^{\circ}$ domains or to current injection, both of which reduce the polarization in the ferroelectric film and as a consequence lead to information loss. ${ }^{11,99,100}$ Two important electrical characterization methods for the development of FeFETs are shown in Fig. 16. A prominent feature in a $C$ - $V$ curve of a MFIS diode is a hysteretic behavior which rises from the ferroelec-
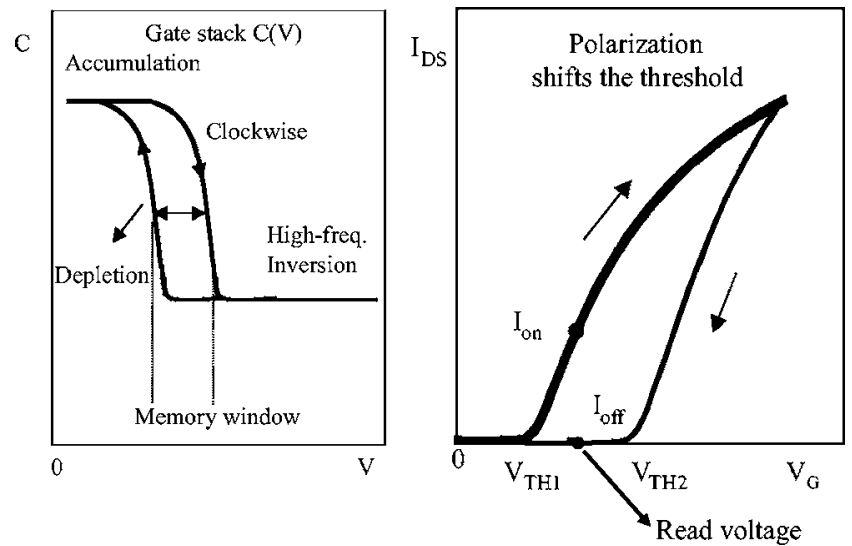

FIG. 16. Upper view graph: $C-V$ characteristic of a semiconductor MFIS diode. The memory window is a result of the ferroelectric hysteresis. Lower view graph: source-drain current $I_{\mathrm{DS}}$ vs gate voltage $V_{G}$ when the gate stack compromises a ferroelectric material. The difference of the threshold voltages $V_{\mathrm{TH} 2}-V_{\mathrm{TH} 2}$ defines the memory window. A possible readout voltage to distinguish both states is shown. tric material. Because uncompensated traps at the insulator/ semiconductor interface can also lead to hysteric $C$ - $V$ curves carefully performed measurements are mandatory for attributing a hysteresis in a $C$ - $V$ curve unambiguously to ferroelectricity. A necessary but not commensurate condition is a clockwise cycle of the hysteresis. The hysteresis of the $C-V$ curve reappears in the source-drain current versus gate voltage curve, as shown in Fig. 16 (lower graph). The maximum voltage width of the hysteresis is defined as the memory window, one of the most important parameters of a FeFET. A possible readout voltage to distinguish the two possible states is also shown in Fig. 16.

Ferroelectricity is found in various material classes and is not restricted to complex oxides alone. Polyvinylidene fluoride (PVDF) copolymer, for example, exhibits ferroelectric properties. ${ }^{101}$ By considering the fast-paced development of polymer electronics in general it is likely that ferroelectric polymers can play a vital role in this exciting field. An interesting approach is the incorporation of ferroelectric polymers as gate oxides in FeFETs. Ferroelectric polymers are deposited at room temperature and therefore interdiffusion is negligible and buffer layer may be not needed. Experiments have been performed on Si substrates ${ }^{102-104}$ and recently entire organic field effect transistors have been successfully built. $^{105-107}$ This could be an important step towards a cheap and flexible nonvolatile memory. On the other hand the switching time will be by far lower than in the case of oxide FRAMs and therefore the market targeted will be different.

\section{Ferroresistive storage}

During the last years there has been an interesting ongoing debate about whether or not ferroresistive materials or the so-called ferroelectric Schottky diodes are an alternative to ferroelectrics for nonvolatile RAMs (NVRAMs). ${ }^{108-110}$ The term ferroresistive means the following: At first a ferroelectric is a perfect insulator. Any kind of leakage current is considered as a parasitic effect, which reduces the device performance of FRAMs in many respects (power consumption, switching, retention, etc.).

On the other hand if we assume a coexistence of a (slightly) conductive film (band conductance) and ferroelectricity it may be possible to alter the resistance between two resistance states by changing the polarization direction of the ferroelectric by applying an external field. We wish to point out that this ferroresistive RAM (FRRAM) has a nondestructive readout and scaling into the gigabit range is possible with a planar technology because remnant polarizations of $1 \mu \mathrm{C} / \mathrm{cm}^{2}$ or less are sufficient. The device resistance shows two (low and high) states, depending on the polarization direction, which represent the logical "0" and "1" with a nondestructive readout. ${ }^{111}$ The appropriate doping and the kind of current transport through the device remain unsolved. Recent simulations show that polar media exhibit electrondipole scattering and lead to very low mobilities. ${ }^{112}$

Another interesting approach is to use so-called ferroelectric tunnel junctions (FTJs). ${ }^{113-119}$ This concept is based on the idea that direct tunneling through ultrathin ferroelectric tunnel barriers depends on the polarization state and is shown schematically in Fig. 17. The concept of a ferroelec- 


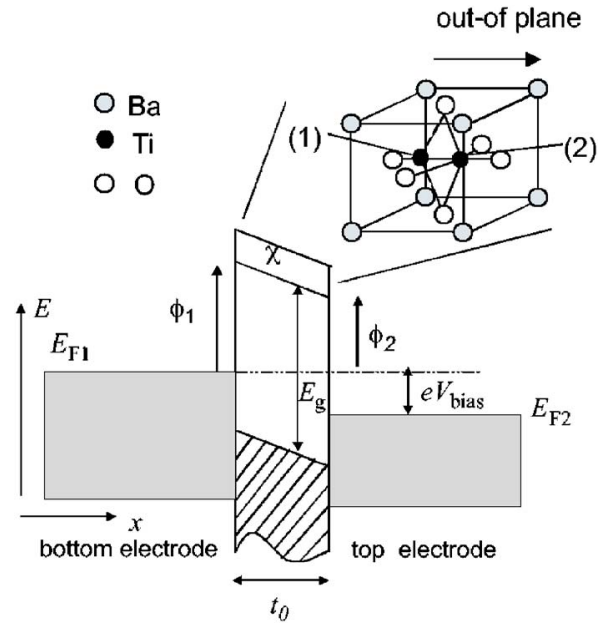

FIG. 17. Sketch of the simplified band structure of a ferroelectric tunnel junction. The unit cell represents the ferroelectric $\left(\mathrm{BaTiO}_{3}\right)$ tunnel barrier. The two possible polarization states (related to the position of the $\mathrm{Ti}$ atom) are labeled by (1) and (2). $E_{F}$ is the Fermi energy, $\chi$ is the electron affinity of the ferroelectric, $t$ is the barrier thickness, and $\phi_{1}$ and $\phi_{2}$ are the barrier heights at the bottom and top electrodes, respectively.

tric tunnel junction is strongly related to the size effect in ultrathin ferroelectric thin films. This topic is described in detail in Sec. III D. Indeed, several phenomena such as strain, microscopic interface effects, and incomplete screening of the ferroelectric bound charge may lead to a resistive switch. ${ }^{120}$ These effects are summarized schematically in Figs. 18(a)-18(c). As an example for the electrostatic origin of resistive switching effect in a FJT, the bound charges of the ferroelectric and for the screening charges a possible situation is shown in Fig. 19(a). In case of incomplete screening under short circuit conditions, a finite depolarization field exists in the ferroelectric. The spatial extension of the free charge is defined in a semiclassical approach by the ThomasFermi screening length and is in the order of $0.1 \mathrm{~nm}$. The potential $\varphi$ (due to the incomplete screening of the polarization charge) (19b) modifies the contact potential of the tunnel junction. The reversal of the polarization results in resistive switching at the coercive voltage $V_{c}$. The shape of the potential is shown in Fig. 19(b) for both polarizations (see solid and dotted lines). In the dependence of the properties of the interface either a symmetric (left $I-V$ in Fig. 19) or an asymmetric (right $I-V$ in Fig. 19) $I-V$ curve can be expected. The resistive switching effect will be large for asymmetric interfaces because here the mean barrier height alters and the tunneling current is modified strongly. Further details about FTJs can be found in Ref. 120.

In principle ferroelectric resistive switching is an interesting approach. Nonetheless, parasitic effects such as local conductivity and electrochemical contribution have to be eliminated to get full advantage of this concept. ${ }^{121,122}$

\section{Scanning probe microscopy (SPM) for multiprobe mass storage}

There has been tremendous growth rate of the areal density of hard disk drives (HDDs). ${ }^{123}$ The areal density has increased by almost by 100\% per year and exceeded $200 \mathrm{Gbits} /$ in. $^{2}$ in 2005 (news release on Hitachi web page
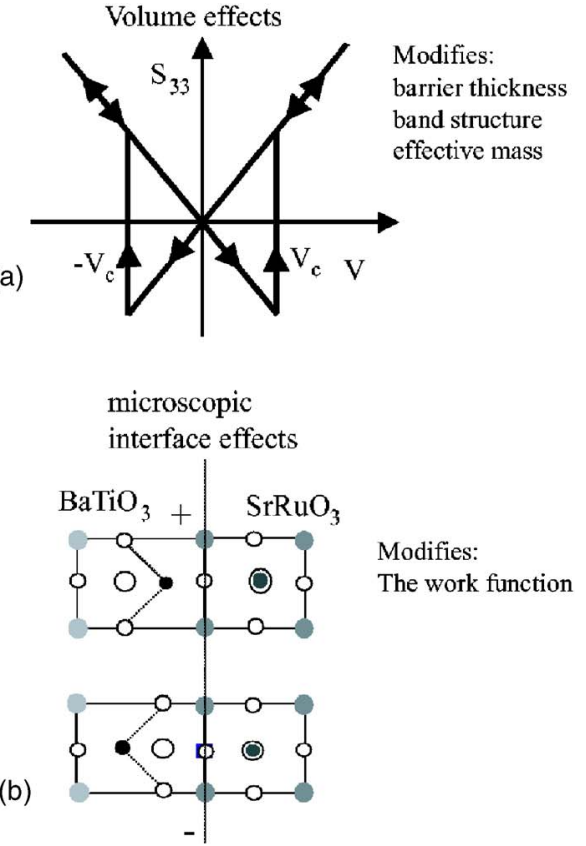

Screening effects

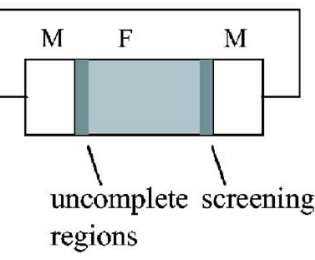

Modifies: the contact potential

(c)

regions

FIG. 18. Possible effects on the tunneling current due to the ferroelectric nature of the tunnel barrier. (a) The strain vs voltage curve (butterfly curve) may modify the thickness of the barrier and therefore the band structure; (b) the local position of the oxygen atoms and the Ti atoms may lead to a modified work function of the adjacent metal in dependency of the polarization (only one interface is shown; (c) screening effects due to incomplete screening of the bound charge by free carriers result in a finite depolarization field. Consequently the contact potential will be modified.

$230 \mathrm{Gbits} /$ in. $^{2}$ with perpendicular recording). This incredible success has came from a dramatic improvement in magnetic (read and write) head technology [for example, the invention of the giant magnetoresistance (GMR) effect ${ }^{124}$, the development of magnetic recoding media (antiferromagnetic coupled media), and a constant decrease in the grain size of the magnetic media. All together this has led to the reduction in cost per bit (as one of the most important criteria) for each generation of HDDs. In the HDD roadmap an area density of several hundred Gbits/in. ${ }^{2}$ is foreseen for 2010 . Nonetheless, in recent years the areal density increase was slowed down significantly to about $20 \%$ increase per year and the question arises: Will there be a showstopper in the areal density increase for magnetic disk drive technology in the future? Indeed the superparamagnetic limit is considered as a serious bottleneck in the coming years, which may limit the maximum storage density of HDDs. With decreasing the grain size of the magnetic recording layers the energy barrier between the two magnetization states becomes comparable to the thermal energy $\left(k_{B} T\right)$ and this will lead to unwanted spontaneous switching of the magnetic bits. Based on current 
Charge distribution in an FTJ: symmetric case

(a)

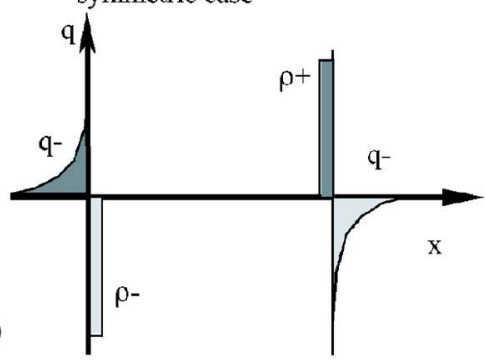

(b)

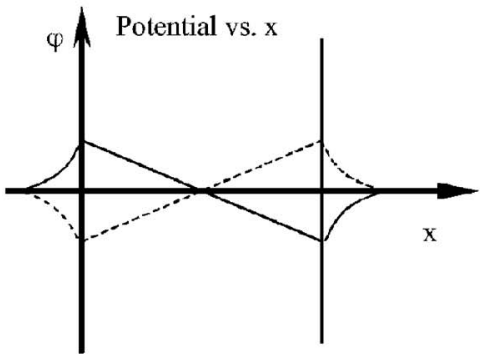

(c)

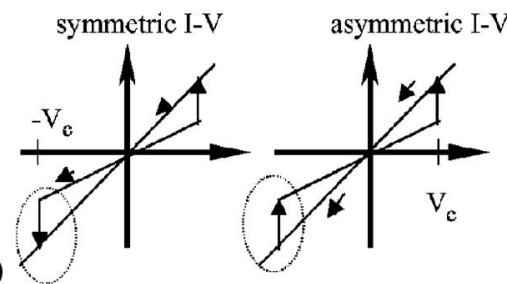

FIG. 19. Charge distribution at the two MF interfaces of a FTJ (a). The potential is shown in (b), the solid and dotted lines show how the potential changes when the polarization points to the right and left, respectively. In (c) the two possible $I-V$ curves including the resistive switching events are shown. The left $I-V$ curve is for a symmetric interface case [i.e., a potential as shown in (b)], whereas the right $I-V$ shows an asymmetric case. The dotted circles highlight the fundamental difference between both curves.

technology, theory predicts a superparamagnetic limit of $9 \mathrm{~nm}$ for spheric grains and could be even lower for materials as FePt. On the other hand, heat-assisted magnetic recording will probably extend this limit. The absolute limit of the maximum area density for HDDs is difficult to predict and we can pose a second, principle question: Why do researchers believe that ferroelectric media may have an even higher potential for mass storage than magnetic media? Eventually two fundamental reasons are important.

(1) The strong (quantum mechanical) exchange coupling of spins in magnetic media leads to rather broad domain walls in the range of $10 \mathrm{~nm}$ and larger. Due to the strong spin coupling the dumping angle from spin to spin is energetically limited and therefore nature tends to rotate the magnetization $\left(180^{\circ}\right)$ in small portions and consequently on a long distance (broad domain wall is needed). For this reason magnetic media consist of finegrained magnetic media, which get more and more difficult to produce with shrinking grain size. In ferroelectric materials the coupling is described on the basis of long-range dipole interactions. This coupling energy is much lower than in magnetic media resulting in very thin domain walls (a few lattice cells). Hence, ferroelec- (a)

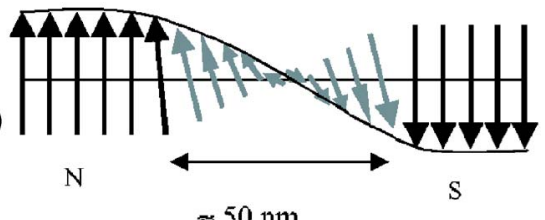

(b)

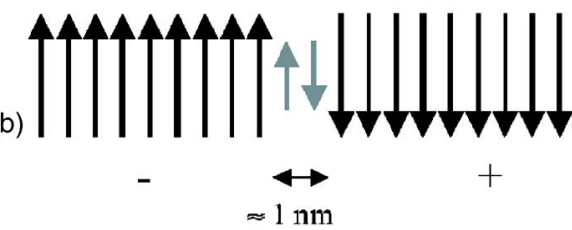

FIG. 20. (a) View graph of a $180^{\circ}$ domain wall (Bloch wall) in a ferromagnet. The arrows present the magnetization directions of the spins. This type of wall is rather larger $(>10 \mathrm{~nm})$. (b) Sketch of a ferroelectric domain wall. The arrows present here the polarization. These walls are known to be thin - in the range of a few unit cells $(1 \mathrm{~nm})$.

tric media will not necessarily consist of fine-grained media for mass storage applications. The situation for a magnetic and a ferroelectric domain wall is illustrated in Figs. 20(a) and 20(b).

(2) In contrast to magnetism, ferroelectricity has the advantage that free charge (electrons, ions, and dipoles) can screen the polarization and reduce or suppress the depolarization field.

From the first argument, a smaller bit size for ferroelectric materials is expected. A clever use of the second issue can lead to the stabilization of the monodomain state of a ferroelectric bit. This will lead to better retention times.

In the following we focus on the most advanced concepts and experiments researching ferroelectric domains for nanometer scale high-density storage. In the current literature there is a clear trend for not transfering the principles of magnetic HDD technology to the building of ferroelectric hard disks. Instead, probe-based storage systems, with arrays of individual cantilevers to write and read the data, are definitely preferred for ferroelectric mass memories. ${ }^{125,126}$ In Fig. 21, the schematic of such a probe-storage system is

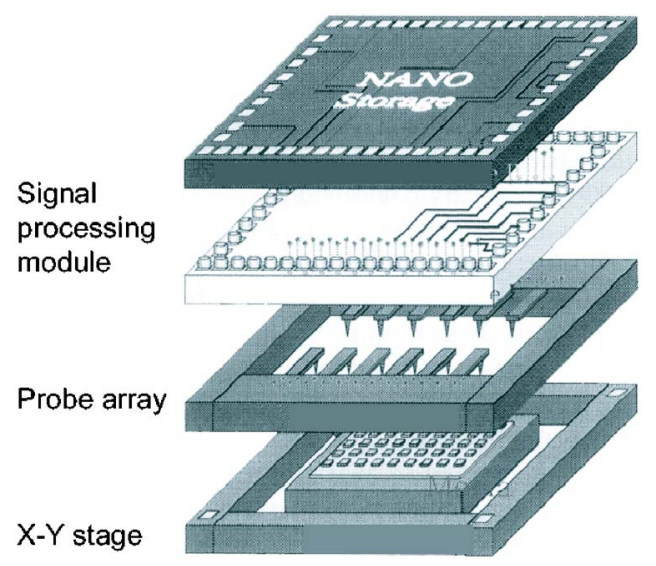

FIG. 21. Principle of a millipede system which compromises a signal processing module, a probe array (AFM needles), and an $x-y$ stage (Ref. 130). 
$\mathrm{x}, \mathrm{y}, \mathrm{z}$ Deformation signals

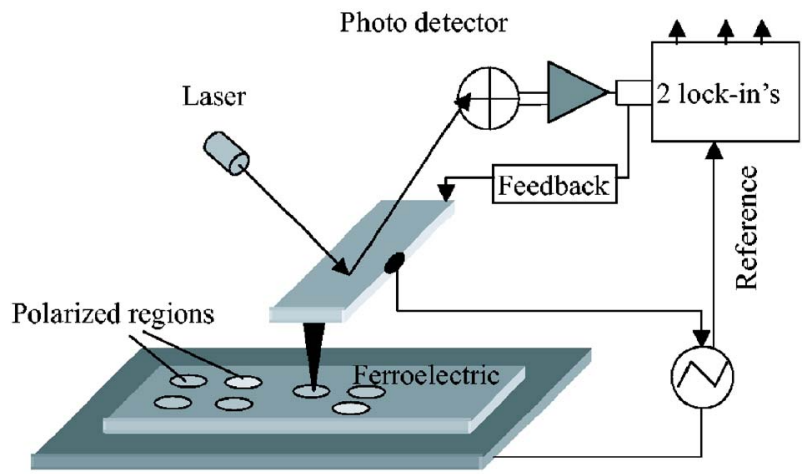

Electrode, e.g. $\mathrm{SrRuO}_{3}$ or $\mathrm{Pt}$

FIG. 22. Experiment of a piezoresponse system (SFM) to detect the deformation of the cantilever in $x, y$, and $z$ directions, a feedback loop, and an ac-reference generator.

shown. The basic features are a movable $x-y$ stage and a multiple cantilever array including a data processing module. At a first glance this approach seems to be rather fragile and not suitable for real products such as smart cards or mp3 players but here are some reasons why probe stages attract so much interest. In principle for hard disks with target values of areal density of $1.5 \mathrm{Tbits} / \mathrm{in}^{2}$ a rotation speed of more than $10.000 \mathrm{rpm}$ is needed for sufficiently short read cycles. Mechanical problems and too high a power consumption are difficult to overcome. Moreover the principles of multiprobe storage have been successfully developed within the millipede project at IBM. In this approach IBM used small indentations in polymer films to store the binary information. Thousands of cantilevers in parallel were employed to form indentations by heating the individual cantilever. The longterm stability of the indentations in the polymer material seems to be the most serious problem for this polymer based probe storage. In addition to the polymer concept several other groups are pursuing probe-based storage using a variety of materials such as phase change materials and magnetic thin films. They are using a method similar to heat-assisted magnetic recording, where a nonlocal magnetic field is applied to the entire material and just the areas that are locally heated with the tip switch.

Ferroelectric thin films are promising candidates. SPM's are the most widely used tools for studying the capabilities of the different media. In the case of ferroelectric materials a voltage pulse is applied to the conductive cantilever of the SPM to initiate local switching of domains on the nanometer scale. There are two elegant methods for reading back the polarization direction in the ferroelectric thin film. One method is detecting the stray field of the individual domains by means of electrostatic force microscopy or by monitoring the local converse piezoelectric response with the aid of a modified SPM setup. Today a deep knowledge exists about the cantilever-ferroelectric surface interaction, for both writing domains in the nanometer scale and data reading. Here we summarize some of the most promising probe-storage concepts.

The principle of domain writing and reading in ferroelectric films is schematically shown in Fig. 22. ${ }^{125-127}$ A con-

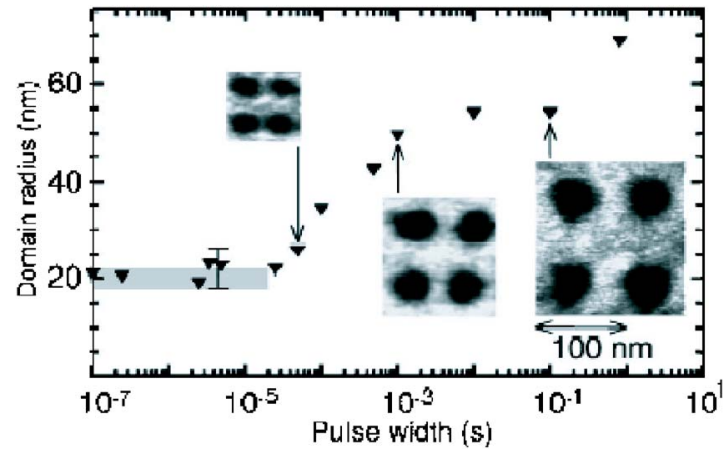

FIG. 23. Example of small dots and the domain radius vs pulse duration width. This figure was taken from Ref. 128.

ductive AFM needle is placed, for example, in contact with a $\mathrm{PbZr}_{x} \mathrm{Ti}_{1-x} \mathrm{O}_{3} / \mathrm{SrRuO}_{3}(\mathrm{PZT} / \mathrm{SRO})$ double layer. The SRO serves as a bottom electrode and PZT represents the ferroelectric film. By applying short voltage pulses between the tip and the SRO electrodes and simultaneously scanning the surface, domains in the form of lines and dots can be written. Hereby the sign of the bias voltage defines whether the polarization vector points from or to the plane of the PZT film. In epitaxial films ultrasmall areas have been polarized and switched by conductive AFM. An example for a series of ferroelectric bits in the form of domains is shown in Fig. $23 .^{128}$ By optimizing the pulse duration, the film thickness and the voltage amplitude area density of $40 \mathrm{Gbits} / \mathrm{cm}^{2}$ have been achieved by Tybell et al. ${ }^{129}$ The ferroelectric film in this example is a (preferred) perpendicular storage medium. The electric field $E$, between the bottom electrode and the nanometer sharp tip, is not simply $E=V / t$, where $t$ is the PZT film thickness. Surface contaminations as well as the unique geometrical configuration lead to sophisticated switching events. In principle thinner PZT films should result in smaller domains and higher storage densities. ${ }^{130}$

There is a family of various AFM related techniques used to investigate the ferroelectric domains, summarized under the generic term "scanning probe microscopy" (SPM) which are suitable to read the programed data (from positive " 1 " or negative " 0 ") of polarized domains. SPM has been applied very successfully to the study of domains in selfassembled ferroelectric dots in the framework of the superparaelectric limit. ${ }^{11}$ Here we focus on the most relevant approaches for ferroelectric mass storage, i.e., electrostatic force gradient microscopy (EFM), piezoresponse force microscopy (PFM), and scanning nonlinear dielectric microscopy (SNDM). An excellent review by Kalinin and Bonnell can be found in Refs. 125 and 126.

During noncontact electrostatic imaging in the EFM mode, the electrostatic force between the dc biased tip and the ferroelectric surface leads to a variation of the cantilever resonant frequency, which is proportional to the force gradient. By using additional topographic information, the surface domain structure can be constructed. ${ }^{125,126}$ While EFM is based on electrostatic interactions, a pure piezoresponse force mode makes use of the electromechanically properties of the ferroelectric. Birk et al. proposed in 1991 the PFM mode as a possible way of detecting the local polarization 


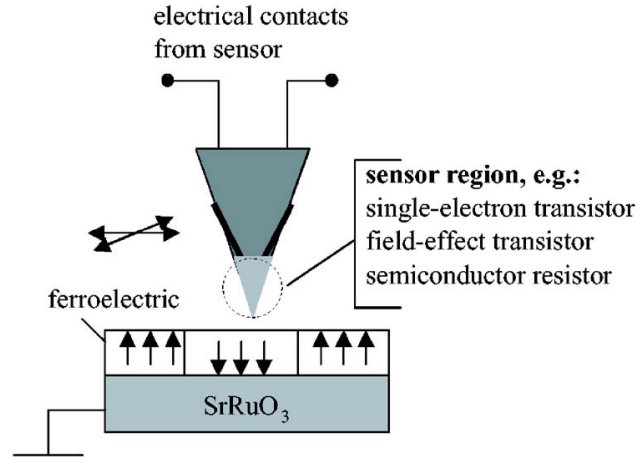

FIG. 24. Cartoon of local sensors at the tip side.

state of a ferroelectric film. ${ }^{131}$ The biased induced surface deformation detection is key for domain imaging in PFM. Here the tip is in contact with the ferroelectric film. The mechanical deformation due to the piezoactivity of the film under bias is measured by the first-harmonic component of bias-induced tip detection (see Fig. 22). It is possible to distinguish between domains with a positive and negative polarization, which is the precondition for storage. Care must be taken because the PFM mode is not only superimposed by the desired electromechanical contribution but also by electrostatic contribution and nonlocal capacitive components. Which part is most important depends, among others, on the strength of the tip-surface contact (indentation depth). ${ }^{125,126}$

Typical EFM and PFM setups for research studies are equipped with a laser reflection system (e.g., laser and four quadrant photodetector) to detect the cantilever deformation. Those systems are very suitable when the investigation concentrates on one cantilever. For probe stages with hundreds of needles, this bulky approach is not appropriate. Possible solutions will be presented at the end of this section.

Cho et al. have developed over the last few years another interesting approach, the SNDM mode. ${ }^{125,126,132}$ The principle of SNDM is the detection of the lowest order of the nonlinear dielectric constant $e_{333}$. The sign of $e_{333}$ changes in accordance with the inversion of the polarization. Electronically the SNDM contains a $L C$ resonant circuit and a conductive needle to measure the capacitance under the tip. The ratio of the alternating variation of the capacitance $\Delta C(t)$ to the static one (without time dependence) leads to a determination of $e_{333} . e_{333}$ is a third rank tensor, like the piezoelectric constant, so that indirect detection of the polarization direction is possible. It is interesting to note that SNDM is a purely electrical method without an optical readout system. Cho et al. have demonstrated area densities as high as 1.5 Tbits/in. ${ }^{2}$ on $\mathrm{LiTaO}_{3}$ single crystals and a domain size as small as $6 \mathrm{~nm} .{ }^{132}$ Although these are encouraging data, the SNDM approach has to also show that it is capable of working with an array of needles.

Especially for the above described EFM technique there are interesting solutions to this problem. Multifunctional local probe sensors, integrated into the individual tips of an array of needles, are able to detect the induced charge at the ferroelectric film surface. In Fig. 24, several possible detectors are summarized. Under ambient conditions the ferroelectric bound charge is compensated by charges from the air.
Therefore the sign of the screening charge (opposite to that of the underlying bound charge) is detected. ${ }^{125}$ There is no decrease of information due to this effect.

Independent of the particular SPM technique, thin epitaxial ferroelectric films on epitaxial electrodes (for example, $\mathrm{SrRuO}_{3}$ ) are mandatory for high density recording. Substrates such as $\mathrm{SrTiO}_{3}$ are ideal from a research point of view but not from a financial one. Fortunately considerable progress has been achieved in depositing high quality $\mathrm{SrTiO}_{3}$ buffer layer on $\mathrm{Si}$ substrates, especially by $\mathrm{MBE}$. $\mathrm{Si}-\mathrm{SrTiO}_{3}$ wafers are considered to be a cheap and reliable platform for $\mathrm{SrRuO}_{3} / \mathrm{PZT}$ single crystal films for mass storage applications.

\section{Conclusion}

Besides the FRAM there exist several alternatives for RAMs as well as for mass storage. For the FeFET severe interface issues between $\mathrm{Si}$, the buffer and/or ferroelectric have to be overcome and/or unconventional concepts have to be developed. Otherwise, the FeFET device will be an interesting research subject but nothing more. ${ }^{133}$

FRRAM devices either with semiconducting ferroelectrics or advanced concepts based on ferroelectric junctions may lead to high integration densities without the necessity of applying sophisticated three-dimensional (3D) conformal coverage techniques as in the case of conventional FRAMs. Mass storage with multiprobe read/write (R/W) heads is currently leaving the laboratory environment and is being seriously considered as an advanced memory technology. Whether this approach will replace the hard disk drive in the future is speculative but not impossible.

\section{MATERIALS AND STRUCTURE}

The initial viable ferroelectric material used in thin film applications was PZT. Over the last years additional materials have found applications. In particular, the choice of materials for FRAM has undergone revolutions and modifications. Section III A deals with selection of materials for ferroelectric memories. Then, in Sec. III B, the attention is focused on domains and domain patterns in thin films, firstly reviewing some highlights of experimental results in the literature and then discussing the theory of domains in thin films. In Sec. III C, epitaxial growth of thin films is discussed in the context of strains in the films since the latter are known to influence strongly the properties. Finally, in Sec. III $\mathrm{D}$, a discussion is carried out on the question of a size limit to ferroelectricity, which is a long-standing question with recent answers by both experimentalists and theoreticians.

\section{A. Selection of materials for ferroelectric memories}

The initial viable ferroelectric material utilized for modern FRAM was the PZT solid solution. The properties of PZT are a strong function of the selected $\mathrm{Zr} / \mathrm{Ti}$ ratio, and the initial choice of composition was the so-called "morphotropic boundary" composition. This refers to compositions with $\mathrm{Ti} / \mathrm{Zr}$ ratios around 52/48, where the polycrystalline materials are relatively easier to switch into a "poled state" with 
high net remanent polarization. It was subsequently recognized that given the high fields that could be applied at moderate voltages in thin films, compositions near the morphotropic boundary were not necessary. Thus, compositions with $\mathrm{Ti} / \mathrm{Zr}$ ratios between $60 / 40$ and $70 / 30$ rapidly became the norm, as they resulted in ferroelectric capacitors with higher switchable polarization and more clearly defined switching voltages.

Around 1991, the layered perovskite $\mathrm{SrBi}_{2} \mathrm{Ta}_{2} \mathrm{O}_{9}(\mathrm{SBT})$, a second material, came into vogue. There was a debate about the preferred choice for FRAM-SBT or PZT: The advantages (and disadvantages) of each material fell in different dimensions. For example, PZT displays the larger remanent and switchable polarization values, but is subject to "polarization fatigue" with repeated switching when used in combination with the preferred electrode material of the time-platinum. In contrast, SBT displays no apparent fatigue on Pt electrodes, and can be processed to operate with lower switching voltages, but has significantly lower switchable polarization charge than PZT. In addition, it displays two processing disadvantages: the biaxial nature of the polarization vector implies that the preferred crystal growth direction (001) can yield ferroelectric capacitors with no switching in the required field direction. Additionally, process temperatures required for crystallization of sufficiently high quality films are significantly higher $\left(100-250{ }^{\circ} \mathrm{C}\right)$ than those of PZT. While not a problem for laboratory scale processing or low device density, the higher temperature does result in a compatibility problem with underlying materials connecting the ferroelectric capacitor to the transistor if one is producing devices with high device density. ${ }^{134}$ Thus the debate is ultimately driven by one's perspective of the future. If one's emphasis is upon high device density and the longer-term scaling to small capacitor dimensions, then process integration and the higher switching charge become dominant, and PZT becomes the natural choice. For lower device densities and higher process temperatures, either material will suffice.

A major concern in the commercialization of FRAM has been the reliability of the integrated ferroelectric capacitor. While much of the scientific discussion around capacitor reliability has centered on the issue of polarization fatigue mentioned above, the industrial companies producing or developing FRAM do not view this as the primary mechanism controlling device reliability. They view this as a problem with a number of acceptable solutions, including the use of oxide electrodes to minimize fatigue. ${ }^{135,136}$ Instead, the phenomenon of "imprint" or the time/temperature-dependent development of a preferred polarization orientation is viewed as a far more significant problem, one that appears to impact all of the thin film ferroelectric materials. While the phenomenon has been studied ${ }^{137-140}$ there does not yet appear to be agreement regarding the underlying mechanisms. Research into alternative ferroelectric materials continues to be quite important, even if progress to date has been limited. In 1999, Park et al. reported an alternative material system based upon the well-known ferroelectric $\mathrm{Bi}_{4} \mathrm{Ti}_{3} \mathrm{O}_{12} \cdot{ }^{141}$ Bismuth titanate films themselves had proved difficult to process successfully, typically yielding films with values of switchable polarization considerably lower than that expected from single crystal data. Park et al. reported on the substitution of lanthanum and neodymium into the $A$ site of bismuth titanate, yielding materials [termed "bismuth lanthanum titanate" (BLT) and "bismuth neodymium titanate" (BNT)] with larger remanent polarization values and lower processing temperatures than SBT, although at the expense of an increased coercive field. The improvements, however, were not large enough to cause a widespread shift to this material system from either PZT or SBT.

Integration onto silicon is generally accomplished in a way that utilizes the ferroelectric in a polycrystalline form, although recent advances in synthesis of epitaxial ferroelectrics on silicon are promising. ${ }^{142}$ Nonetheless, as pointed out above, the SBT films utilized in commercial production are currently randomly polycrystalline: by necessity, as-textured SBT typically grows in an inappropriate orientation. PZT films incorporated in devices, on the other hand, are more typically grown fiber textured, usually (111), but occasionally (001). Texturing may reduce capacitor-to-capacitor variation under certain circumstances ${ }^{143,144}$ and can otherwise yield improved ferroelectric switching.

Both epitaxial ferroelectric films can also of course be deposited on appropriate single crystal substrates. The Japanese ferroelectrics community, in particular, has been very active in the exploration of epitaxial orientation effects for the different ferroelectric materials systems. For example, using a carefully controlled MOCVD process, Saito and coworkers have compared the properties of epitaxial films of PZT, SBT, BLT, and BNT for different orientations. ${ }^{145,146}$ It is interesting to note that the $P-E$ hysteresis loops of PZT films, in particular, can be very similar to those expected for bulk single crystals, with square hysteresis loops and low coercive fields. ${ }^{146,147}$ Particularly striking are the films with low $\mathrm{Ti} / \mathrm{Zr}$ ratios, on the side of the morphotropic boundary expected to have rhombohedral symmetry, as there is marked contrast between the epitaxial and the polycrystalline (or textured) films. The polycrystalline films with low Ti contents and rhombohedral symmetry always show "sloped" (or nonsquare) hysteresis loops, in which the switching voltage is not well defined. This at a minimum implies that a high operating voltage is not only required but also portends nonideal switching (for example, less mobile ferroelastic domain walls). If the square loops of the epitaxial rhombohedral films could be retained in a manufacturable process and integrated with $\mathrm{Si}$ wafers and a Si-based front end, this could well be the "ideal" or at least a preferred ferroelectric thin film material for FRAM.

There does not appear to be a concerted effort to develop the square-looped rhombohedral PZT films that have been tantalizingly demonstrated, as discussed above. This may be a difficult objective, as strain may well play a role. The previously described films of Foster et al. ${ }^{147}$ were on $\mathrm{SrTiO}_{3}$ substrates, and strain here may have helped to ensure the ideal orientation of the polarization normal to the substrate. Thus, necessary strain control may be difficult to achieve on the $\mathrm{Si}$ substrates used for memory devices which have a small thermal expansion coefficient relative to the perovskite oxides. 
Ceramic PZT used for piezoelectric applications always contains substantial concentrations of dopants and site substitutions, primarily to control properties through the modification of domain wall mobility. ${ }^{148}$ The ferroelectric thin film community has not followed this path to the same extent, presumably because the substitutions do not ostensibly have a major impact on switching, as measured via polarization-field hysteresis loops. However, improved diagnostics for fast switching behavior are now becoming available, ${ }^{149-151}$ and this may allow a more fruitful study of dopant and substitution effects on fast switching. Furthermore, the mechanisms of imprint may need to be revisited in the context of dopant additions.

\section{B. Domains in thin films}

\section{Observations}

Domain patterns in ferroelectric thin films have became an issue of interest over the past decade. The most investigated are domain structures in (001) oriented thin films of tetragonal ferroelectric perovskites. These structures have been attracting much attention of workers since the early 1990s, though some observations of domain patterns in this kind of films have been reported in yet earlier papers. ${ }^{152}$ Activity has been mainly centered on $\mathrm{PbTiO}_{3}$, and to a lesser extent on films of $\mathrm{Pb}(\mathrm{Zr}, \mathrm{Ti}) \mathrm{O}_{3}(\mathrm{PZT}),(\mathrm{Pb}, \mathrm{La}) \mathrm{TiO}_{3}$ (PLT), $\mathrm{BaTiO}_{3}$, and $(\mathrm{Ba}, \mathrm{Sr}) \mathrm{TiO}_{3}$ (BST). To date, most of the studies have been performed on epitaxial films deposited onto crystalline substrates of (001) or pseudo-(001) (Ref. 448) orientations $\left(\mathrm{MgO}, \mathrm{KTaO}_{3}, \mathrm{SrTiO}_{3}\right.$, and $\left.\mathrm{LaAlO}_{3}\right)$ using a wide variety of deposition techniques. The domain patterns in films are visualized using different techniques. The most conventional techniques are scanning force microscopy (SFM) pioneered in ferroelectric thin films by Franke et $a l^{127}$ and transmission electron microscopy (TEM). In earlier papers a good domain contrast was achieved by using a method which included chemical etching followed by the making of platinum-carbon replicas, the latter being visualized using an electron microscope. ${ }^{152,153} \mathrm{~A}$ high resolution of $180^{\circ}$ domains was recently demonstrated using scanning nonlinear dielectric microscopy (SNDM). ${ }^{154}$ An important parameter for domain patterns - the fraction of each type of the domains that make the pattern — can be reliably determined by comparing intensities of $\mathrm{x}$-ray diffraction (XRD) peaks corresponding to different domain states. Using all these techniques a large body of experimental data, sometimes contradictory, have been collected on the domain features of (001) thin films of tetragonal ferroelectric perovskites.

Ferroelectric materials are also generally ferroelastic. Therefore, domain relationships are typically characterized by the approximate angle by which the polarization rotates across a domain wall, and whether or not there is a difference in the orientations of the spontaneous strains for two parts of the domain pair, i.e., whether the domain wall is ferroelastic as well as ferroelectric.

The principle difference between the film on a substrate and a mechanically free sample is that the former is strained and clamped by the substrate. This leads to breaking of the
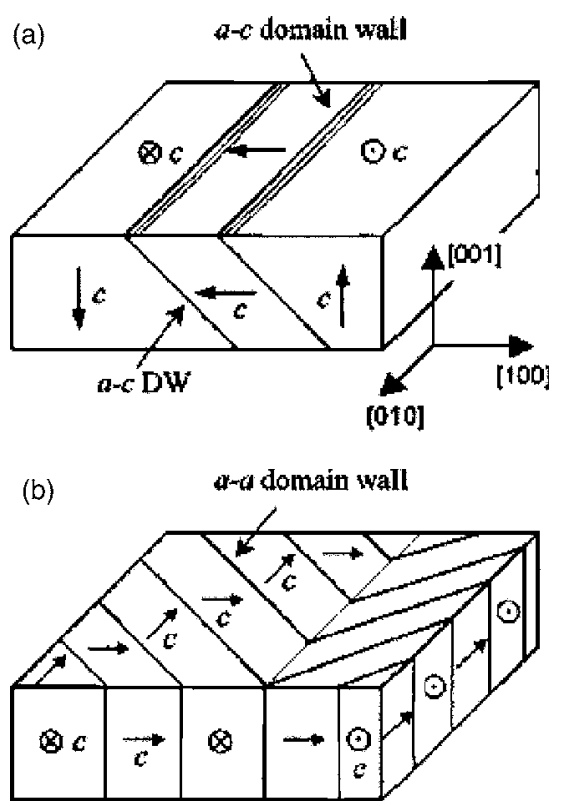

FIG. 25. Domain patterns in (001) tetragonal ferroelectric thin films: $a / c$ pattern (a) and $a_{1} / a_{1}$ pattern (b).

films into ferroelastic domains. One reasonably expects that domain patterns in the film should be formed by walls that meet the conditions of mechanical compatibility of the bulk material. In the case of ferroelastic domains in tetragonal ferroelectric perovskites, walls of $\{110\}$ orientations are the only ones expected. In a (001) film, this determines the two simplest types of ferroelastic patterns made of $c$ and $a$ domains where the $c$ or $a$ axes are normal or nearly normal to the plane of the film, respectively. These are the so-called $a / c$ and $a_{1} / a_{2}$ variants. The orientations of the walls and spontaneous polarization in the $a / c$ and $a_{1} / a_{2}$ variants are illustrated in Fig. 25. A general feature of the experimentally observed patterns is that $a_{1} / a_{2}$ variants contain equal fractions of $a_{1}$ and $a_{2}$ domains in accordance with simple theoretical arguments whereas the fraction of $c$ domains in $a / c$-domain patterns is very sensitive to many parameters of the film/substrate system.

The domain patterns experimentally observed in (001) thin films of tetragonal ferroelectric perovskites are $a / c$ and $a_{1} / a_{2}$ variants as well as their superpositions. Figure 26

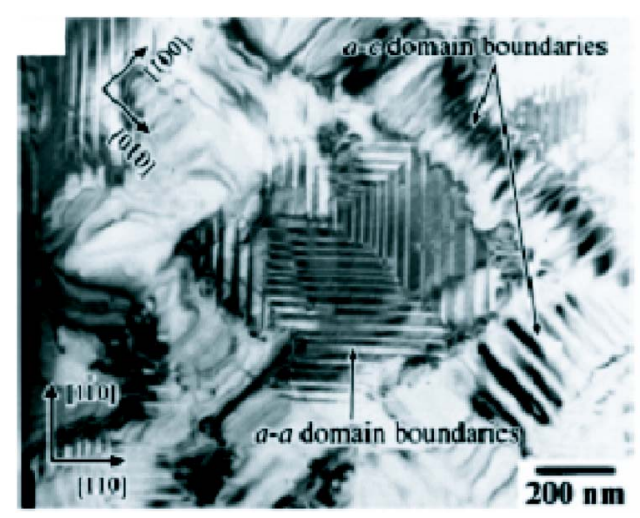

FIG. 26. Coexistence of different types of domain patterns in (001) tetragonal ferroelectric thin films. Plan-view TEM image of a epitaxial $\mathrm{PbTiO}_{3}$ films, after Ref. 446. 


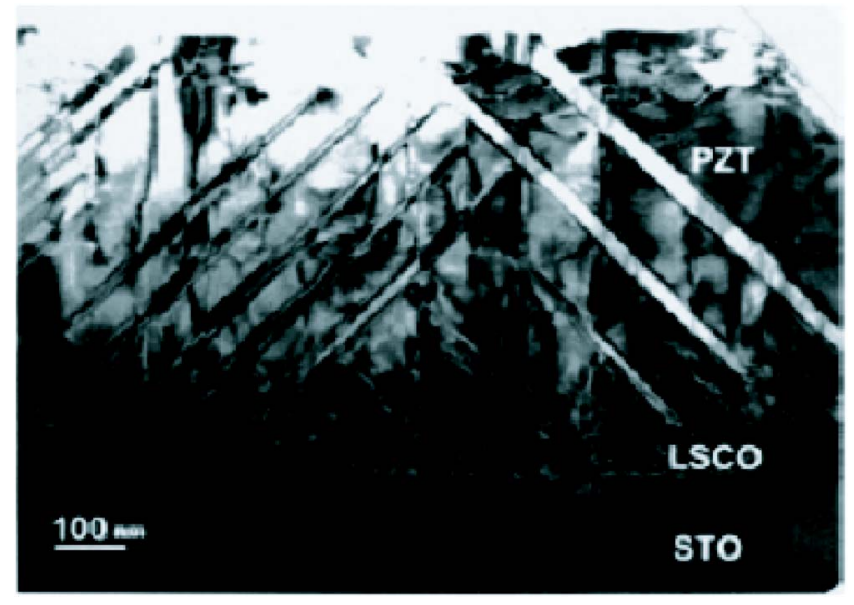

FIG. 27. Cross-section TEM image of a $\mathrm{Pb}\left(\mathrm{Zr}_{0.2} \mathrm{Ti}_{0.8}\right) \mathrm{O}_{3}$ epitaxial film, after Ref. 155 .

shows a plan-view TEM image of $200 \mathrm{~nm}$ thick $\mathrm{PbTiO}_{3}$ film (on $\mathrm{KTaO}_{3}$ ) where two $a_{1} / a_{2}$ variants and at least two $a / c$ variants are seen. The coexistence of $a_{1} / a_{2}$ and $a / c$ variants is a rare situation; more typical is the coexistence of only $a / c$ variants (two or more). An example of such a coexistence is illustrated in Fig. 27. Here a cross-section TEM image of a 400 nm thick PZT (20/80) film (on $\mathrm{SrTiO}_{3}$ substrate) shows two $a / c$ variants with the walls parallel to the (101) and (101) planes. ${ }^{155}$ A SFM image obtained from an identically processed film is shown in Fig. 28. A cross-hatched pattern shown in this figure corresponds to interpenetrating $a / c$ variants having walls parallel to (101) and (011) planes, the black stripes visualizing the intersections of $a$ domains with the free surface of the film. Similar images have been obtained by different authors using various visualizing techniques: SFM in the height mode, ${ }^{156}$ SFM in the piezoelectric mode, ${ }^{157}$ TEM, ${ }^{155}$ and chemical etching followed by making platinum-carbon replicas. ${ }^{153}$ Actually these structures should be considered as made up of all three possible ferroelastic

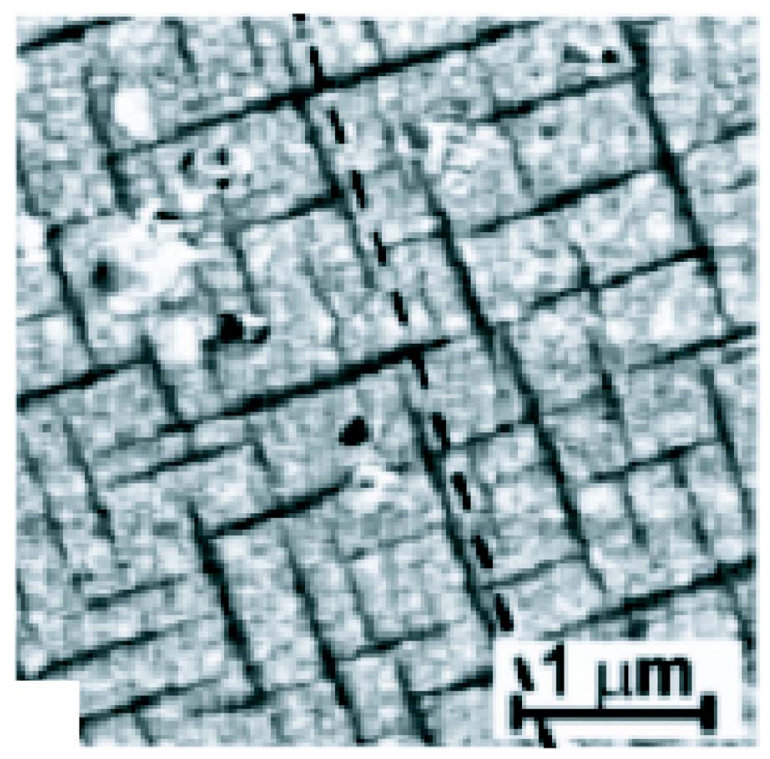

FIG. 28. Typical piezoelectric image of a $\mathrm{Pb}\left(\mathrm{Zr}_{0.2} \mathrm{Ti}_{0.8}\right) \mathrm{O}_{3}$ epitaxial film, after Ref. 155.

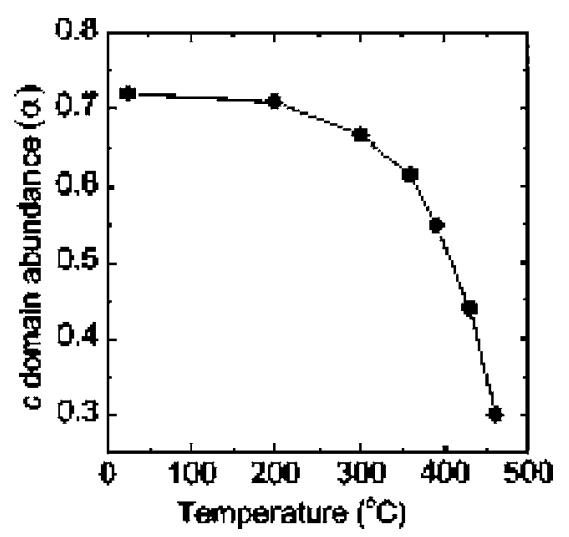

FIG. 29. Temperature dependence of the fraction of $c$ domains in an epitaxial (001) $\mathrm{PbTiO}_{3}$ thin film on $\mathrm{MgO}$, after Ref. 167.

domain states; they have been called three-domain-state structures. ${ }^{158}$ Independently of whether an $a / c$ domain pattern corresponds to a three-domain-state structure or whether the film consists of nonoverlapping areas containing different types of $a / c$ variant, the films contain nearly equal fractions of four possible $a$-domain states. If the film is not too thin, these states can be distinguished by using XRD intensity reciprocal space mapping. ${ }^{159,160}$

The images shown above suggest that the domain patterns in the films can be characterized by a typical period. However, it is seen from these figures that, in different regions of the same film, the period can readily differ by a factor of 2. Despite this scatter of the data a clear trend can be traced: the average period of the domain pattern in films of a given material increases with increasing film thickness.

The most clearly determined characteristic of $a / c$-domain patterns is the fractions of its different domain states. A convenient parameter describing the composition of the pattern is the volume fraction of $c$ domains, which we denote as $\alpha$. It can be evaluated from TEM or SFM images. For instance, using the TEM image shown in Fig. 27 one can evaluate $\alpha=0.7-0.9$ from the ratio of the volumes of the $a$ and $c$ domains shown in this image. Another way to evaluate the average fraction of domain states in a pattern is to analyze the intensities of the XRD peaks.

For $\mathrm{PbTiO}_{3}$ and PZT films, the fraction $\alpha$ has been evaluated as a function of film thickness $h$ by many authors. It has been found that the $\alpha(h)$ dependence is sensitive to the substrate material and film composition. Available experimental data reveal both increasing $\left(\mathrm{MgO}\right.$ and $\mathrm{KTaO}_{3}$ substrates $)^{160,161}$ and decreasing $\left(\mathrm{SrTiO}_{3}\right.$ and $\mathrm{LaAlO}_{3}$ substrates) ${ }^{162,163}$ types of the $\alpha(h)$ dependence. In these experiments, there is a correlation between the sign of the film/ substrate misfit strain in the paraelectric phase and the sense of the $\alpha(h)$ dependence.

The temperature dependence of $\alpha$ has also been experimentally addressed for films of $\mathrm{PbTiO}_{3}$ and PLT on $\mathrm{MgO}$ (Refs. 164-167) and for $\mathrm{PbTiO}_{3} / / \mathrm{KTaO}_{3}$ films. ${ }^{166}$ The same trend, namely, a reduction of $\alpha$ with increasing temperature, was obtained in these papers. The results of Lee and Baik ${ }^{167}$ for $\mathrm{a} \mathrm{PbTiO}_{3} / / \mathrm{MgO}$ film $(h=300 \mathrm{~nm})$ are shown in Fig. 29.

Data on the concentration dependence of the fraction of $c$ domains $\alpha$ in $\mathrm{PZT} / / \mathrm{MgO}$ system have been reported by 
Lee et al. ${ }^{168}$ It was found that, both at room and phase transition temperatures, $\alpha$ increased with increasing $\mathrm{Zr}$ concentration, the effect being better pronounced at the transition temperature. Similar behavior has been reported for the Laconcentration dependence of $\alpha$ in PLT//MgO films. ${ }^{165}$

All experimental data on $a / c$ patterns in (001) films presented above have been obtained for modified $\mathrm{PbTiO}_{3}$ films. Much less information is available on $a / c$-patterned ferroelectric thin films of $\mathrm{BaTiO}_{3}$. Despite considerable amount of publications on (001) $\mathrm{BaTiO}_{3}$ films, information about their ferroelastic domain patterns is virtually absent since most works report on $a$ or $c$ monovariant states. A correlation between the type of monovariant and the thermal expansion coefficient of the substrate has been reported for $\mathrm{MgO}$, GaAs, and fused quartz substrates. ${ }^{169,170}$ For the $\mathrm{MgO}$ substrate, which has a higher coefficient of thermal expansion than that of $\mathrm{BaTiO}_{3}$ in the paraelectric phase, the $c$-domain state was observed, whereas for GaAs and fused quartz, the opposite situation takes place and the $a$-domain state was observed. As for the $a / c$ pattern, it has been only observed in the peripheral regions of $\mathrm{BaTiO}_{3}(001)$ films. The $a_{1} / a_{2}$ pattern was reported for $\mathrm{BaTiO}_{3}(001)$ films $(h=500-600 \mathrm{~nm})$ by Kaiser et al. ${ }^{171}$

Antiparallel domain patterns have been observed in (001) perovskite thin films using different experimental techniques. The information on $180^{\circ}$ domains in these systems was reported by Surowiak et al. ${ }^{172}$ who employed chemical etching followed by the making of platinum-carbon replicas. These authors monitored antiparallel $c$ domains in $\left(\mathrm{Ba}_{1-x} \mathrm{Sr}_{x}\right) \mathrm{TiO}_{3} / / \mathrm{MgO}(x=0-0.3)$ films obtained by sputtering covering the thickness range $h=20-5000 \mathrm{~nm}$. The mean width of the observed domains was reported to increase as $\approx 0.28 h$ with increasing film thickness $h$.

Thickness-dependent $180^{\circ}$ domain patterns in $\mathrm{BaTiO}_{3} / / \mathrm{SrTiO}_{3}$ films have been reported by Tsunekawa et al. ${ }^{173,174}$ for film thicknesses of 4, 20, and $1000 \mathrm{~nm}$. The information was obtained by using a tapping mode SFM. A correlation between the sign of polarization in domains and their height, which enabled the visualization of the pattern, was attributed by the authors to dry etching effects. The end shape of the $180^{\circ}$ cylindrical domain was found to be regular (almost round) in thinner films becoming irregular with increasing thickness. In thicker films, the coexistence of $180^{\circ}$ and $90^{\circ}$ domains was observed. The determined thickness dependence of the average diameter of the domain ends $D$ was described by a relation $D=3.2 h^{0.51}$ ( $D$ and $h$ are in nanometers). Similar correlation between the domain height and their sign was reported by Odagawa and Cho ${ }^{154}$ who used a combination of height-mode SMF technique with scanning nonlinear dielectric microscopy. In their experiments, the polarity of the domains was determined from the phase of the second polarization harmonics taken simultaneously with surface topology images. For $\mathrm{PZT} / / \mathrm{SrTiO}_{3}$ films, the positively poled domains were found to be $1 \mathrm{~nm}$ higher than those poled negatively: typically $1-3 \mathrm{~nm}$ domains have been found separated with $0.3-0.5 \mathrm{~nm}$ domain boundaries.

All the experiments discussed above deal with information obtained from the interface or from a thin interfacial layer of the films. To obtain direct information about $180^{\circ}$ domain patterns in the "bulk" of the film one should test the sample using radiation that can penetrate into it. An example of such a study has been offered by Streiffer et al. ${ }^{175}$ In this work, epitaxial $\mathrm{PbTiO}_{3} / / \mathrm{SrTiO}_{3}$ films of thicknesses $h$ $=1.6-42 \mathrm{~nm}$ were studied using x-ray scattering. Upon cooling below the transition temperature, satellites appeared around Bragg peaks indicating the presence of $180^{\circ}$ stripe domain patterns of period $W=3.7-24 \mathrm{~nm}$, the thickness dependence of period of the patterns being consistent with the square-root law $W=3.2 h^{0.5}$ ( $W$ and $h$ are in nanometers).

Domain patterns with oblique orientations have rarely been studied. Only the situations in (001) rhombohedral and (111) tetragonal thin films of ferroelectric perovskites have been discussed and, specially, the case of perovskite ferroelectrics with a cubic $m \overline{3} m$ paraelectric phase and tetragonal $4 m m$ and/or rhombohedral $3 m$ ferroelectric phases. The common feature of these systems is that none of their domain states has their spontaneous polarization purely normal to the plane of the film; the vectors of spontaneous polarization in these domain states are arranged in a symmetric manner with respect to the film normal.

The geometry of domain patterns in these systems has been treated by Streiffer et al. ${ }^{176}$ and Romanov et al. ${ }^{177}$ based on the conditions of mechanical and electrical compatibilities. Specifically, it has been found that in these systems, in both domains, the components of the spontaneous polarization normal to the wall are equal whereas their in-plane components are equal in absolute value but differ in sign.

For the case of the cubic-rhombohedral ferroelectric phase transition $(m \overline{3} m \Rightarrow 3 m)$, four ferroelastic states (or variants) are possible, being separated by walls of $\{100\}$ and $\{110\}$ orientations. Two types of patterns are possible: Domain patterns of the first type contain walls that are perpendicular to the plane of the film. The net polarization of this pattern possesses only the in-plane component. Domain patterns of the second type contain oblique $\{110\}$ walls that make angles of about $45^{\circ}$ with the plane of the film. The net polarization of this pattern possesses a nonzero out-of-plane component. Thus, the domain structure with vertical walls is nonpolar in the out-of-plane direction, whereas the structure with oblique walls is "perfectly poled" in this direction, i.e., it exhibits the maximal vertical projection of the net spontaneous polarization.

In the case of (111) tetragonal films one finds a situation similar to that discussed above for (001) rhombohedral films. Two types of patterns are possible. One is nonpolar in the out-of-plane direction and contains vertical walls, which can be parallel either to $(\overline{1} 01)$, or to $(0 \overline{1} 1)$, or to $(\overline{1} 10)$ crystallographic planes. The patterns of the second type are perfectly poled in the out-of-plane direction and contain oblique walls of either (101), or (011), or (110) orientations.

Comparing the two types of the domain patterns in the rhombohedral and tetragonal systems discussed above, an essential qualitative difference between these systems can be pointed out. In (001) rhombohedral films, the intersection lines between domain walls and the upper interface are oriented either along [100] or along [010] directions disregard- 
ing the type of the pattern. These directions are equivalent in terms of the cubic symmetry of the paraelectric phase. For this reason, one cannot distinguish the "nonpolar" and "perfectly poled" patterns merely judging from the orientation of these lines. In contrast, in (111) tetragonal films, for the nonpolar and perfectly poled patterns, the orientations of the intersection lines are nonequivalent in the aforementioned sense. Thus, the information on the cross section of a domain pattern with the film interface enables an identification of the type of pattern.

A comprehensive investigation of the domain structure of (100) rhombohedral PZT thin films has been performed by Streiffer et $a l .{ }^{176}$ The results of plan-view TEM and scanning electron microscopy (SEM) studies were reported for nonelectroded films of two compositions (80/20 and 65/35). Using the information from $\delta$-fringe contrast, plan-view TEM tilting experiments, and arcing of higher-order diffraction spots in selected area electron diffraction pattern it was shown that the domain structure of the films contains exclusively nonpolar patterns having walls of (100) and (010) orientations. For $520 \mathrm{~nm}$ thick PZT 80/20 films the domain spacing was found to be $80-120 \mathrm{~nm}$, and for $700 \mathrm{~nm}$ thick PZT 65/35 films 50-100 nm. Vertical orientation of the domain walls in nonelectroded (100) rhombohedral PZT films has also been documented by TEM cross-section images by Lin $e t a l .{ }^{178}$ As was pointed out by Streiffer et al. ${ }^{176}$ the nonpolar domain configuration can be anticipated for nonelectroded films since it is more electrostatically favorable compared to the alternative perfectly poled pattern.

It is important to note that some deposition methods such as sputtering create a bias field on a growing film, perhaps favoring polar domain patterns; other deposition methods such as metal-organic chemical vapor deposition, chemical solution deposition, and molecular beam epitaxy do not and, thus, may favor nonpolar domain configurations.

Experimental data on domain patterns in (111) tetragonal ferroelectric thin films are limited even though (111) tetragonal PZT films are of high interest for application in nonvolatile memory devices. Results of SFM (topography mode) studies of (111) PZT (25/75) films deposited on Si covered with different sublayers $\left(\mathrm{Pt}, \mathrm{IrO}_{2}\right.$, and $\left.\mathrm{Al}_{2} \mathrm{O}_{3}\right)$ have been reported by Zybill et al. ${ }^{179,180}$ For $700 \mathrm{~nm}$ films, the patterns observed have a typical domain spacing of about $20 \mathrm{~nm}$. For films on Pt sublayer, the spacing increases with increasing film thickness $h: h=300,500$, and $700 \mathrm{~nm}$ correspond to spacings of 12,16 , and $19 \mathrm{~nm}$, respectively. ${ }^{180}$ The investigated films were found to contain a perfectly poled pattern with oblique domain walls. An example of nonpolar domain pattern in a (111) PZT tetragonal ferroelectric film has been reported by Romanov et al. ${ }^{177}$

Experimental information available in the literature on the domain patterns in systems different from tetragonal and rhombohedral perovskite films is very limited as well as is relevant theoretical work.

The room temperature domain structure of orthorhombic $\mathrm{KNbO}_{3}$ films has been experimentally addressed by Gopalan and Raj. ${ }^{181,182}$ The studied films had thicknesses of about $350 \mathrm{~nm}$ and were laser ablated onto either a $\mathrm{SrTiO}_{3}(100)$ substrate or a $\mathrm{MgO}(100)$ substrate with a $12 \mathrm{~nm} \mathrm{SrTiO}_{3}$ sublayer. The films exhibit excellent cube-on-cube epitaxy. TEM investigations reveal that among 12 possible ferroelectric domain states only 8 , which have nonzero out-of-plane component of the spontaneous polarization, participate in the domain patterns. Judging from the plan-view TEM micrographs the films were found to contain a domain pattern made up of $60^{\circ}$ and $120^{\circ}$ domain walls whose traces on the

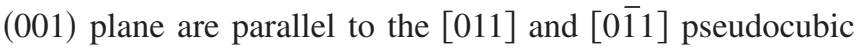
directions, with the typical domain spacing being about $100 \mathrm{~nm}$. Area fractions of four domain pairs (with the same in-plane component of the spontaneous polarization within any pair) were evaluated from the result of the second optical harmonics generation measurements. For some $30 \mathrm{~mm}^{2}$ of area of the as-deposited films tested by the incident beam, the fractions of the pairs were found to be virtually equal (to within a $2 \%-3 \%$ accuracy). As similar characterization of monoclinic $\mathrm{Bi}_{4} \mathrm{Ti}_{3} \mathrm{O}_{12}$ films is offered by Barad et al. ${ }^{183}$

Films of isostructural Aurivillius phase $\mathrm{SrBi}_{2} \mathrm{Ta}_{2} \mathrm{O}_{9}$ and $\mathrm{SrBi}_{2} \mathrm{Nb}_{2} \mathrm{O}_{9}$ have been drawing the attention of researcher because of the use of these materials in memory applications. However, the information on domain patterns in these films is very limited. A weak piezoelectric response and very small ferroelastic distortions make standard TEM and SFM visualization of domain pattern in these materials difficult. The results obtained by optimized SFM (Ref. 184) and Fourier transform high-resowtion transmission electron microscopy ${ }^{185}$ (HRTEM) suggest the presence of quite irregular domain patterns with typical spatial scale of some $10-100 \mathrm{~nm}$. According to these results, in films of these materials, both $180^{\circ}$ and $90^{\circ}$ domain walls are strongly curved.

\section{Theory}

There are two basic reasons for the breaking of ferroelectric films into domains: mechanical clamping by the substrate and the depolarizing effect. The former effect leads to formation of ferroelastic domains; the latter mainly leads to the formation of nonferroelastic $180^{\circ}$ domains. Mechanical clamping by the substrate is of key importance for the formation of domain patterns in the films, whereas the effect of the depolarizing field is associated with smaller energies and is often of secondary importance. The main body of theoretical work on domain patterns in thin films has been centered on the mechanical clamping effects. The problem of domain formation driven by elastic effects in thin film ferroics was addressed by Roytburd ${ }^{186}$ and later treated by many workers. These studies have made available a good qualitative and, in many cases, quantitative mathematical description of the phenomenon. The progress has been achieved via four main approaches: (i) consideration of relatively dense domain structures (the domain period smaller than the film thickness) using the average mechanical energy of the systems as the basic approximation, which we will call the mean strain approach; ${ }^{186}$ (ii) calculations based on analytical results for elastic Green's functions; ${ }^{187}$ (iii) calculations using fictitious dislocations for the description of the deformation fields; ${ }^{188,189}$ and (iv) Landau theory of dense domain patterns. ${ }^{190,191}$ 

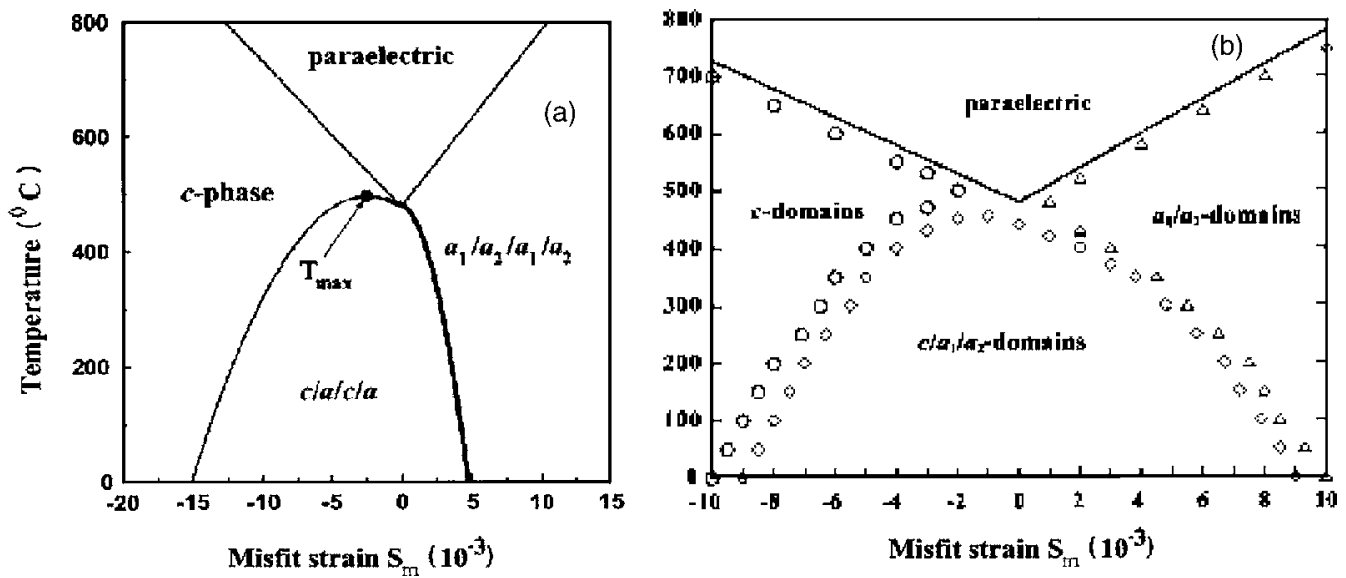

FIG. 30. Misfit-strain/temperature phase diagrams for (001) $\mathrm{PbTiO}_{3}$ thin films calculated by Koukhar et al. (Ref. 190) and Li et al. (Ref. 196).

Roytburd's mean strain approach, though being relatively simple mathematically, provides a good insight into the phenomenon and, in most cases, gives an adequate quantitative description. It is fully justified if the domain structure is dense, i.e., when the domain period $W$ is smaller than the film thickness $h$. Using this approach the mechanical energy per unit area of a film can be presented as the elastic energy corresponding to the average (macroscopic) strain in the system, the energy of microstresses at the film/substrate interface, the energy of the domain walls, and the contribution related to the difference in the energy of the film/substrate interface for the different domain states. The approach leads to a square-root dependence of the pattern period $W$ on the film thickness $h$. Applied to the case of tetragonal (001), it enables calculation of the fraction of $c$ domains, $\alpha$, in the pattern as a function of the material parameters of the ferroelectric, the ferroelectric/substrate misfit strain, and the film thickness (the substrate is considered much thicker than the film). ${ }^{192,193}$

The Roytburd approach has two principle limitations. First, it is not applicable to the case of very thin films where the domain spacing is larger than the film thickness. Second, it is not applicable close to the transition temperature where the energy associated with the order parameter (neglected in the Roytburd approach) becomes comparable to the mechanical energy of the system. A theory dealing with nondense domain pattern has been developed in terms of more advanced methods in elasticity theory. ${ }^{187-189}$ The results obtained confirm those by the Roytburd approach in the range of its applicability, which for the classical case of $\mathrm{PbTiO}_{3}$ films corresponds to films thicker than $10 \mathrm{~nm}$. The advanced analysis has revealed a qualitative feature for the ultrathin films: in the case of appreciable tensile misfit strain the $a_{1} / a_{2}$ variant is favorable at any film thickness; the Roytburd theory predicts $a$ variant (single domain state) for films thinner than a certain critical thickness. Theories for the domain pattern, which take into account the order parameter energy, have been developed for the case of dense domain patterns by Koukhar et al. ${ }^{190}$ and Pertsev and Koukhar ${ }^{194}$ in terms of analytical Landau theory and by Li et al. ${ }^{191,195,196}$ in terms of the so-called phase-field numerical approach. Figure 30 shows the misfit-strain temperature phase diagram for domain states in (001) $\mathrm{PbTiO}_{3}$ films obtained in Refs. 190, 191, and 196.

Comparison of the theory with experiment can be performed for the case of $(001) \mathrm{PbTiO}_{3}$ films. In this system, room temperature experimental dependence of the fraction of $c$ domains in the $a / c$ pattern, $\alpha$, agrees reasonably with the results of the theory by Koukhar et al. ${ }^{190}$ as illustrated in Fig. 31. It is instructive to mention that for this problem, the difference between the predictions of this theory and of that by Roytburd ${ }^{192,193}$ is vanishingly small. ${ }^{197}$ The theoretical results by Koukhar et al., ${ }^{190}$ are comparable with the experimental data on the temperature dependence of $\alpha$ by Lee and Baik ${ }^{167}$ for $\mathrm{PbTiO}_{3} / / \mathrm{MgO}$ films, which is shown in Fig. 29. The finite element analysis ${ }^{198}$ has also provided an adequate modeling of this dependence. At the same time, the Roytburd theory predicts a temperature dependence that is too weak. ${ }^{197}$ The experimental thickness dependence of $\alpha$ reported by Lee and $\mathrm{Baik}^{160}$ in (001) $\mathrm{PbTiO}_{3} / / \mathrm{MgO}$ films can be interpreted in term of Roytburd theory with the thickness dependence of the misfit strain taken into account. However, the observed thickness dependence of $\alpha$ (Refs. 164-166) is often difficult

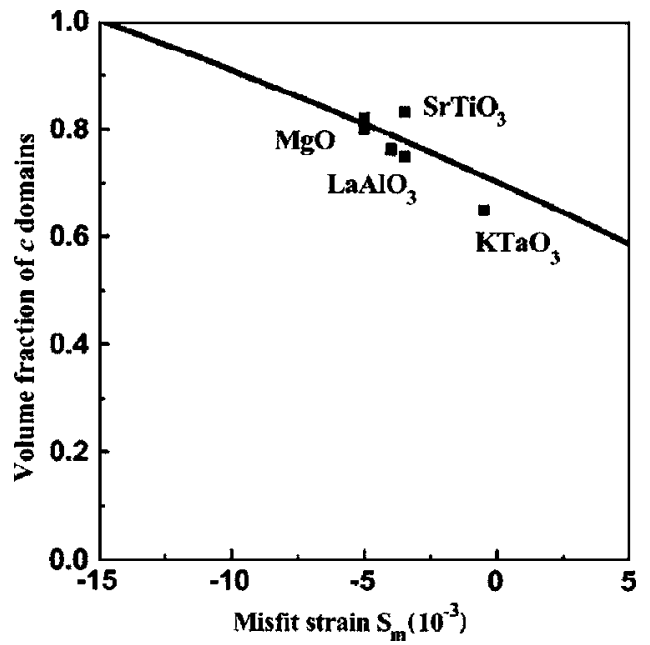

FIG. 31. Misfit-strain dependence of the fraction of $c$ domains in $a / c$ pattern in (001) $\mathrm{PbTiO}_{3}$ thin films at room temperature. Points-experimental data for different substrates; line-theory (after Ref. 190). 
to interpret in terms of the existing theories. The problem is probably associated with the difficulty in the theoretical description of the thickness dependence of the stress release. At the same time, the correlation between the sense of the dependence $\alpha(h)$ and the sign of the film/substrate misfit strain can be rationalized in terms of existing theories. Data on the domain spacing $D$ in the ferroelectric domain pattern for PZT and $\mathrm{PbTiO}_{3}$ films can be extracted from many experimental papers. All of those correspond to the situation where the square-root law $D=g \sqrt{h}$ is expected to be valid with the constant $g$ moderately temperature and material dependent. An attempt to analyze this body of experimental data in terms of the square-root law yielded values of $g$ which spread over a few orders of magnitude. ${ }^{197}$ This possibly implies that, in films of typical thickness, the energy controlling the pattern period is much smaller than the pinning energy, so that the real domain pattern is far from the equilibrium pattern to which the law $D=g \sqrt{h}$ is applicable.

The problem of nonferroelastic $180^{\circ}$ domain patterns in thin films has been addressed in the context of the impact of a passive layer by Kopal et al. ${ }^{199,200}$ and of an insulating substrate by Streiffer et al. ${ }^{175}$ Readers are also referred to the paper by Stephenson and Elder ${ }^{201}$ in this issue. It has been shown that even in a short circuited capacitor, the presence of a passive layer should lead to a breakup of the films into $180^{\circ}$ domains. In the case of an insulating substrate the situation has been found to be similar to the case of a nonelectroded sample of double thickness where the classical square-root law for the thickness dependence of the domain spacing is applicable. The results of modeling have been found to be in good agreement with the experimental data for $\mathrm{PbTiO}_{3}$ films. ${ }^{175}$

\section{Epitaxial growth and the resulting strain}

Many technological ideas in the processing of high quality epitaxial thin films have been advanced in the past during the development of semiconductive films. In these innovations, chemical vapor deposition and MBE played an important role. The development of high quality epitaxial ferroelectric films (more generally, oxide films) has followed these technologies, adding other suitable technologies for oxide films, such as pulsed laser deposition and chemical solution decomposition.

To explore ferroelectric behavior especially that pertaining to the size effect (see Sec. III D) and the strain, epitaxially grown ferroelectric films are studied. For instance, investigation of the effect of mechanical strain on ferroelectric behavior is effectively achieved using epitaxially grown films as the application of high stress in the order of gigapascal is possible by subjecting the film to the effect of the substrate.

Since residual stress is a function of the thickness of the film, the thickness dependence of the properties of epitaxial films has been used as an experimental way to investigate the strain effect in ferroelectrics. As the epitaxial stresses due to lattice mismatch can be relaxed by the formation of misfit dislocations during the film growth, the lattice constant of the growing film can be described by taking an "effective"
TABLE II. Lattice constant of a class of possible substrate materials: the rare-earth scandates. The lattice parameters $a, b$, and $c$ of the orthorhombic structure as well as $c / 2$ and $\sqrt{a^{2}+b^{2}} / 2$ are listed for the different materials, after Ref. 212.

\begin{tabular}{lccccc}
\hline \hline Material & $a(\AA)$ & $b(\AA)$ & $c(\AA)$ & $c / 2(\AA)$ & $\sqrt{a^{2}+b^{2}} / 2(\AA)$ \\
\hline $\mathrm{LaScO}_{3}$ & 5.678 & 5.787 & 8.098 & 4.049 & 4.053 \\
$\mathrm{CeScO}_{3}$ & 5.787 & 5.626 & 8.047 & 4.023 & 4.036 \\
$\mathrm{PrScO}_{3}$ & 5.770 & 5.602 & 8.010 & 4.005 & 4.021 \\
$\mathrm{NdScO}_{3}$ & 5.770 & 5.579 & 7.999 & 4.000 & 4.013 \\
$\mathrm{SmScO}_{3}$ & 5.524 & 5.750 & 7.953 & 3.977 & 3.987 \\
$\mathrm{EuScO}_{3}$ & 5.502 & 5.750 & 7.954 & 3.977 & 3.979 \\
$\mathrm{GdScO}_{3}$ & 5.488 & 5.746 & 7.934 & 3.967 & 3.973 \\
$\mathrm{TbScO}_{3}$ & 5.466 & 5.727 & 7.915 & 3.958 & 3.958 \\
$\mathrm{DyScO}_{3}$ & 5.440 & 5.713 & 7.887 & 3.943 & 3.944 \\
$\mathrm{HoScO}_{3}$ & 5.427 & 5.714 & 7.895 & 3.947 & 3.940 \\
\hline \hline
\end{tabular}

substrate parameter. ${ }^{202,203}$ Peng et al. have investigated the strain relaxation behavior of $\mathrm{SrTiO}_{3}$ films on $\mathrm{LaAlO}_{3}$ and $\mathrm{MgO}$ substrates during the film growth ${ }^{204}$ and have found a general agreement with the theoretical prediction. However, strain relaxation slows down with the decrease in the growth temperature due to a lower thermal energy that impedes reaching equilibrium state. ${ }^{204}$ Canedy et al. ${ }^{205}$ and Li et al. ${ }^{206}$ in investigating the effect of $\mathrm{Ba}_{x} \mathrm{Sr}_{1-x} \mathrm{TiO}_{3}$ film thickness on the permittivity have found good agreement with the theoretical predictions taking into account the mechanical strain effect, namely, that the reciprocal permittivity is proportional to biaxial strain in the film. ${ }^{205-207}$

Some other ways to control the mechanical strain have also been used by selecting substrates having different lattice constants ${ }^{208}$ and thermal expansion coefficients, by changing deposition conditions such as oxygen pressure during the film growth, by postannealing process, and by inserting a thin layer of a different chemical composition in between the film and the substrate. ${ }^{209}$ One of the onerous experimental problems is the difficulty in controlling the strain of the film while keeping fixed the other parameters, such as the film thickness, interface structure, and chemical composition, in order to avoid these additional influences. However, by using a so-called two-step growth technique, the strain of $\mathrm{SrTiO}_{3}$ films has been systematically controlled in the range of $-0.1 \%$ to $-0.7 \%$ and while keeping the substrate, the total thickness of the film and the deposition condition unchanged. $^{210,211}$ Dielectric measurement of the straincontrolled films showed that the ferroelectric transition temperature varied according to the strain state as expected. Nevertheless, since the control of strain utilizing the relaxation process of the growing film is strongly related to misfit dislocation formation, it is indeed difficult to purely separate the influence of the mechanical strain in the film from other variables.

Recently, the epitaxial growth of ferroelectric films onto highly matching substrates has been used to lower the dislocation density of the films and consequently improve the quality of the films. Schubert et al. ${ }^{212}$ proposed a set of rareearth scandates as substrates for ferroelectric films (see Table II). These materials are orthorhombic at room temperature and are isostructural with $\mathrm{GdFeO}_{3}$. As can be seen in the 
table, $\sqrt{a^{2}+b^{2}} / 2$ and $c / 2$ of these (110) planes lead to a good lattice matching with typical perovskite ferroelectrics. By selecting the appropriate composition for the substrate, the growth of a high quality ferroelectric film can be achieved. ${ }^{212}$ Some of them are commercially available. The additional advantages of these available substrates are (i) they are twinfree unlike the more common $\mathrm{LaAlO}_{3}$ whose phase transition occurs during cooling down from the high deposition temperature for the films and (ii) they have low permittivity which is suitable for the fabrication of the planar capacitor. One remarkable experiment was the growth of $\mathrm{SrTiO}_{3}$ on $\mathrm{DyScO}_{3} \cdot{ }^{208}$ Since the lattice mismatch of this system is small ( $1 \%$ at room temperature), it delays the onset of strain relaxation in the growing film, meaning the critical thickness for misfit dislocation ${ }^{213}$ is greater. The $50 \mathrm{~nm}$ thick $\mathrm{SrTiO}_{3}$ film grown on $\mathrm{DyScO}_{3}$ by $\mathrm{MBE}$ was strongly stretched along in-plane directions and was ferroelectric at room temperature, ${ }^{208}$ although single crystals of $\mathrm{SrTiO}_{3}$ do not show a ferroelectric transition even down to $0 \mathrm{~K}$.

It is also interesting to note that a difference in the surface termination layer of the substrate may affect the strain relaxation behavior of the films. The surface termination of oxide substrates is usually controlled by chemical etching, by thermal annealing of the substrates or by deposition of one atomic layer onto these tailored surfaces according to need. ${ }^{214-216}$ In the case of perovskite $A B \mathrm{O}_{3}$-type substrates, there are two possible surface termination layers, i.e., $A \mathrm{O}$ and $B \mathrm{O}_{2}$ layers. Kim et al. reported that, for the $\mathrm{SrTiO}_{3} / \mathrm{LaAlO}_{3}$ heterostructure, the $\mathrm{AlO}_{2}$-terminated $\mathrm{LaAlO}_{3}$ surface leads to the fast strain relaxation of the growing $\mathrm{SrTiO}_{3}$ film, compared to the LaO-terminated $\mathrm{LaAlO}_{3}$, due to the unfavorable chemical matching of the $(\mathrm{SrO})^{0} /\left(\mathrm{AlO}_{2}\right)^{-}$interface. $^{214}$

\section{Size limits to ferroelectricity in thin films}

One of the longest-standing issues in ferroelectricity is the behavior of the ferroelectric phase transition as the system size is reduced. As ferroelectricity is a cooperative phenomenon, it has long been expected that ferroelectric behavior should be modified near surfaces and interfaces, within some distance determined by the correlation length of the underlying interactions. ${ }^{217-219}$ Depending on the details of the surface or interface interaction, this modification of behavior can be expected to manifest itself as either a decrease or increase in the ferroelectric phase transition temperature $T_{C}$ relative to the unperturbed bulk value as the system size becomes smaller and as surfaces and interfaces make a larger relative contribution to the material response. Generally, $T_{C}$ has been found experimentally to be lower for smaller ferroelectric samples and, thus, these intrinsic size effects have been thought to degrade ferroelectricity.

This straightforward concept of an intrinsic size effect is complicated in practice, however, by the fact that many extrinsic factors also strongly influence the ferroelectric phase transition. These include depolarization resulting from imperfect screening of divergences in the polarization, for screening either by domain formation ${ }^{220}$ or by interfacial compensation charge, ${ }^{221,222}$ nonstoichiometry, impurities, line defects and planar defects, and more general limitations to overall crystal quality, all of which can alter ferroelectric properties; and electromechanical effects, which can shift ferroelectric phase transition temperatures by tens or even hundreds of ${ }^{\circ} \mathrm{C}$, and rescale the various properties which make ferroelectrics so useful. ${ }^{195,223,224}$ Many extrinsic effects are inherently size dependent, or cannot be maintained constant as size is varied. Given the many ways that the ferroelectric phase transition can therefore depend on system size, separation of any intrinsic size effect from the broad spectrum of behavior has provided a stimulating challenge to both experimentalists and theorists.

Much of the experimental effort on size effects in ferroelectrics has centered on the study of the phase transition in powders of various perovskite oxide ferroelectrics as a function of particle size. ${ }^{225}$ While this has been a very fruitful approach, there are advantages to investigating model systems that allow more independent control of size relative to other factors. Thin films, in particular, are very attractive as platforms for investigating size effects, as they can be synthesized with well-controlled strain states (thus allowing a proper accounting of electromechanical effects), with good compositional control and with very high crystal quality, and with thickness $t$ as an easily varied parameter such that system size (at least in one dimension) can be changed while other synthesis parameters are held fixed. The ferroelectric thin film community's ability to synthesize both polycrystalline and epitaxial ferroelectric thin films has advanced considerably in the last decade, with continued progress in chemical solution deposition, ${ }^{226-228}$ physical vapor deposition including pulsed laser ablation, ${ }^{229-232}$ sputtering ${ }^{233-238}$ and molecular beam epitaxy, ${ }^{208,239,240}$ and metal-organic chemical vapor deposition. ${ }^{241-253}$ Additionally, fundamental insight into thin film behavior is becoming increasingly important as technologies utilizing ferroelectric thin films continue to push towards smaller and smaller dimensions. ${ }^{88}$

Because of these continued improvements in ferroelectric film quality, significant advances have been made in exploring the impact of film thickness on ferroelectric behavior both in polycrystalline and in epitaxial films. Several recent reviews have summarized efforts in this area. ${ }^{254-256}$ In general, studies can be subdivided into two different classes: (A) measurement of the ferroelectric transition temperature $T_{C}$ for a film of a given thickness, by tracking a property directly related to the transition as a function of temperature and (B) identification of the film thickness at which a system at a given temperature and strain state ceases to be ferroelectric. In some cases, a property such as spontaneous strain or polarization has been monitored at fixed temperature and extrapolated given known ferroelectric physics to give information on $T_{C}$ for a given thickness. Note that $a b$ initio theoretical methods, which have blossomed in recent years in their ability to yield insight into ferroelectric behavior, ${ }^{254,255,257,258}$ generally fall into this class as they are performed by necessity at zero temperature. One area where it is expected that great progress will occur in the near future is in the continued application to ferroelectric thin films of finite temperature theoretical methods, including molecular dynamics, ${ }^{259}$ and effective Hamiltonian approaches. ${ }^{260}$ Moreover, size ef- 
fects on properties other than $T_{C}$ (such as coercive field) are of great interest to the community. These subjects are complex and are discussed elsewhere in this issue.

While many investigators have explored size effects in polycrystalline films, ${ }^{256,261}$ here we focus on the current state of the art for epitaxial films, as this represents the case where confounding effects are most easily minimized. The system that has been perhaps most studied experimentally is $\mathrm{Pb}\left(\mathrm{Zr}_{x} \mathrm{Ti}_{1-x}\right) \mathrm{O}_{3}$ (PZT) deposited onto [001] $\mathrm{SrTiO}_{3}$, with $x$ chosen from within the tetragonal region of the phase diagram, $0 \leqslant x \leqslant 52$. Epitaxial strain is predicted to increase $T_{C}$ by approximately $260{ }^{\circ} \mathrm{C}$ for perfectly coherent $\mathrm{PbTiO}_{3} / \mathrm{SrTiO}_{3}$ in the absence of any thickness dependence, and by more as $x$ is increased. However, the relevant metric can be recast as $\Delta T_{C}(t)$, thus allowing comparison across different mechanical boundary conditions.

Several reasons account for the popularity of the PZT system. First, it is possible to synthesize very high quality epitaxial $\mathrm{PZT} / \mathrm{SrTiO}_{3}$ heterostructures with the PZT film under compressive strain. This tends to force the ferroelectric polarization to be out of the substrate plane, which is the orientation that is most straightforward to probe by most methods. Furthermore, the PZT ferroelectric phase transition occurs at convenient temperatures in the bulk (although $T_{C}$ is dependent on $x$ ), and its spontaneous polarizations and strains are large.

The beginning of the modern era of understanding thickness effects in epitaxial perovskite films can arguably be placed at the observation of ferroelectricity in $4 \mathrm{~nm}$ $\mathrm{PbZr}_{0.2} \mathrm{Ti}_{0.8} \mathrm{O}_{3}$ deposited by sputtering onto conducting $\mathrm{Nb}: \mathrm{SrTiO}_{3} .{ }^{129,262}$ This was remarkable, in that the critical size for ferroelectricity in the closely related material $\mathrm{PbTiO}_{3}$ was extrapolated to be on the order of $11 \mathrm{~nm}$ based on studies of powders. ${ }^{225}$ As pointed out above, ferroelectricity is very sensitive to incomplete screening of depolarizing fields, ${ }^{221,222}$ and in the work of Tybell et al. ${ }^{129}$ it can be presumed that adequate screening charge was provided by the doped $\mathrm{SrTiO}_{3}$ at the bottom interface. The top surface of the film was screened either by charge provided by the measurement process or by charge from the environment.

Following this work, the first experiments were performed to directly measure $T_{C}$ as a function of epitaxial ferroelectric film thickness, specifically for $\mathrm{PbTiO}_{3}$ deposited onto insulating $\mathrm{SrTiO}_{3}$. These measurements were initially made by using in situ synchrotron x-ray scattering to determine the temperature at which the out-of-plane $\mathrm{PbTiO}_{3}$ lattice parameter displayed a pronounced kink due to the spontaneous ferroelastic strain that occurs at the ferroelectric transition. ${ }^{175}$ However, it was also found that for this system, depolarizing fields were screened by formation of oppositely oriented domains, and that these domains were sufficiently well ordered to yield strong $\mathrm{x}$-ray scattering at reciprocal space positions corresponding to the domain period. The appearance of this scattering was determined to be an excellent indicator $T_{C}$ and was used to establish ${ }^{263}$ a critical thickness for ferroelectricity in this system of three unit cells or approximately $1.2 \mathrm{~nm}$.

Concurrently, others evaluated properties of films of different thicknesses at room temperature, either examining switched polarization in $\mathrm{SrRuO}_{3} / \mathrm{PbZr}_{0.2} \mathrm{Ti}_{0.8} \mathrm{O}_{3} /$ $\mathrm{SrRuO}_{3} / \mathrm{SrTiO}_{3}$ heterostructures ${ }^{264}$ or tetragonality in $\mathrm{PbTiO}_{3} / \mathrm{SrTiO}_{3}$ heterostructures. ${ }^{265}$ In both cases, changes as a function of film thickness can be interpreted in terms of the temperature increment $\Delta T(t)=\left[T_{C}(t)-T\right]$ with respect to a thickness-dependent $T_{C}(t)$. Results from all of these studies are qualitatively consistent, indicating that although $T_{C}$ is markedly reduced in the thinnest films, coherently strained films of the PZT family still demonstrate ferroelectricity down to thicknesses of just a few unit cells.

In hindsight, these results may be less surprising than they first appeared. In contrast to intuition, theoretical investigations have suggested that intrinsic size effects in the absence of depolarization may, in fact, be relatively small. ${ }^{260}$ Furthermore, simulations have strongly indicated that in the case of a ferroelectric sandwiched between electrodes, the major size effect is indeed electrostatic - it is due to residual depolarization arising from imperfect screening by the electrodes. ${ }^{266}$ Still, existing theoretical models do not give quantitative descriptions of the functional form of the experimentally observed thickness dependence for films either on insulating substrates or with electrodes. This opportunity is recognized-theoretical models that better treat depolarization and domain formation in ultrathin ferroelectric films continue to be developed. ${ }^{201,259,267}$

As is apparent here, much is now known about the PZT system and a very interesting additional question is to what extent the behavior observed for this system is common to other families of ferroelectrics. For instance, high quality epitaxial $\left(\mathrm{Ba}_{x} \mathrm{Sr}_{1-x}\right) \mathrm{TiO}_{3}$ films can also be synthesized. However, despite progress in identifying phase transitions in this system, ${ }^{208,268}$ the thickness dependence of these transitions has proven harder to study because of the small spontaneous strains. Other issues that the field will continue to pursue include improving synthesis of the complex multicomponent alloys that are at the heart of ferroelectric materials science, with attention to controlling composition and composition gradients over extremely small length scales; exploring the transitions between stripe domain phases and chargecompensated states; and understanding the detailed atomic structure of interfaces formed between ferroelectric films and other materials beyond the relatively simple systems that have been investigated in detail to date. Extension of what has been learned from ultrathin ferroelectric films to superlattices and other types of integrated heterostructures promises much excitement in the community for years to come.

\section{PROPERTIES AND FUNCTIONALITY}

The properties of ferroelectric thin films and their measurement techniques have been intensively studied during the past decade. We selected for discussion only a small number of topics: the properties related to reliability of the ferroelectric memories are firstly presented since they continue to be important and not yet fully understood. Nanoscale probing techniques for ferroelectric films are yet another obvious choice for review, because of the growing need for these techniques and in order to continue the refinement and innovation in this direction. The choice of including the piezo- 
electric nonlinearity of ferroelectric films in the present paper originates from the belief that this topic will become important in future piezo-MEMS applications. Permittivity and loss in ferroelectric films are not yet well understood, in spite of the importance of these properties to the functioning of any ferroelectric thin-film-based device: Sec. IV D summarizes the state of the art in this topic. A more comprehensive review is given in other papers of this volume.

\section{A. Polarization reversal and related reliability issues in ferroelectric films}

\section{Models for polarization switching}

The switching of spontaneous polarization is an essential property of ferroelectric thin films attracting much attention because of its importance for various applications. Apart from practical issues, the mechanisms and kinetics of switching represent a challenging physical problem and many of its aspects still need to be understood.

In general, the classic switching scenario for thin films involves nucleation followed by needlelike vertical domain growth through the film and their subsequent enlargement due to domain wall lateral movement. In this context, the model describing the switching kinetics first needs to determine whether the limiting stage of switching is the domain nucleation or domain wall movement. The widely accepted switching concept developed by Orihara et $a l .{ }^{269}$ considers polarization reversal in terms of domain wall motion, describing the kinetics of overlapping switched regions based on the formalism of Kolmogorov and Avrami ${ }^{270}$ (the model is called hereafter the KAI model). This approach enables a correct description of switching in ferroelectric bulk materials, but encounters difficulties when applied to ferroelectric polycrystalline thin films. ${ }^{271,272}$ In order to provide an adequate description of switching kinetics for the latter case an alternative model has been proposed. ${ }^{272}$ The basic difference of this model compared to KAI model is that the ferroelectric film is regarded as an ensemble of independent microscopic regions where the switching process develops independently. The nucleation of reversed domains is assumed to be the limiting stage of the switching process, so that instead of kinetics of domain wall motion the model deals with the statistics of domain nucleation. Under these assumptions the switching performance depends mainly on the distribution of nucleation times, which is expected to be quite wide at moderate electric fields. ${ }^{273}$ This model [hereafter referred to as the nucleation-limited switching (NLS) model] predicts switching profiles completely different from the KAI model. The nucleation of reversed domains, when enhanced by defects, should exhibit thermally activated kinetics, except at very low temperatures where "cold field" nucleation has been predicted. ${ }^{273}$

An alternative model exists, which assumes that switching is limited by the vertical propagation of $180^{\circ}$ domains rather than by lateral domain wall motion, suggesting a switching scenario characterized by dagger-shaped domains with walls that make small angles with the polarization direction. ${ }^{274}$
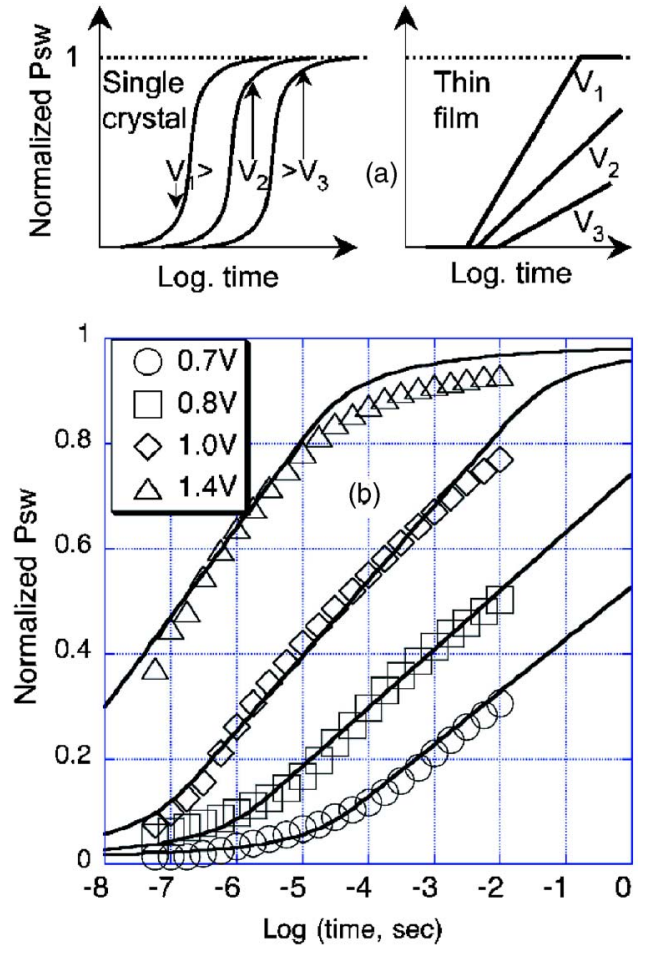

FIG. 32. (a) Schematic curves of normalized switched polarization vs time for different voltages for single crystals (described by the KAI model) and polycrystalline thin films (described by the NLS model); (b) switching curves for $150 \mathrm{~nm} \mathrm{IrO}_{x} / \mathrm{PZT} / \mathrm{Pt}$ ferroelectric capacitors and fitting curves generated using the NLS model (solid lines).

\section{Macroscopic and microscopic experimental evidences for different models}

The macroscopic characteristics of the polarization reversal process have been studied using various techniques, including collecting polarization hysteresis loops ${ }^{275,276}$ or measuring switching curves of polarization versus time at fixed voltage, for different voltages. ${ }^{272}$ In order to understand the switching mechanisms, the latter technique looks preferable since it delivers switching curves that allow a more straightforward interpretation compared to hysteresis loops. The KAI model predicts relatively sharp switching curves, with a normalized switched area that grows from zero to unity within one or two time decades. As the voltage increases, the switching curve shifts along the time axis towards lower times, but its slope remains unchanged. Alternatively, the NLS model predicts a completely different behavior of the switching curves, with the slope strongly changing with voltage [Fig. 32(a)]. At relatively low voltage, the switching process may be very slow, with a steady increase of the switched polarization continuing over many time decades. ${ }^{272}$ Such qualitative difference between predictions of the two models suggests that the polarization reversal mechanism can be identified by switching curve analysis.

It was shown in Ref. 272 that the switching behavior of polycrystalline PZT film capacitors agrees with the NLS model, suggesting that the switching process is limited by nucleation [Fig. 32(b)]. The spectrum of switching times shows a thermal-activation-type behavior within a wide temperature range. ${ }^{277}$ On the other hand, for epitaxial PZT films the switching behavior agrees with the classical KAI model, 
similar to bulk crystals. ${ }^{278,279}$ Such a difference in switching kinetics may be attributed to the conditions of domain growth, which are different for epitaxial and polycrystalline films. An epitaxial film may be switched entirely with a small number of nucleation centers due to the relatively easy lateral movement of the domain walls, whereas for polycrystalline films the domain wall movement may be strongly impeded by grain boundaries and other inhomogenities.

In order to study the switching process at the microscopic scale, piezoelectric scanning probe microscopy is commonly used. ${ }^{280,281}$ To get microscopic evidence for NLS behavior, the independently switched regions in the polycrystalline PZT film sandwiched between Pt electrodes were investigated using this technique. ${ }^{282}$ It was demonstrated that elementary switched regions look like grains or clusters of grains, suggesting a strong role of grain boundaries in the propagation of switched domains. On the other hand, piezoSPM results of epitaxial PZT ferroelectric films show continuous growth of the domains, in agreement with the KAI model. ${ }^{155}$ Piezo-SPM measurements have also been evoked to prove the scenario of switching limited by vertical domain growth. $^{274}$

The mechanism of polarization reversal in ferroelectric films may change with temperature. In particular, polycrystalline PZT films showing NLS-type switching kinetics within the temperature range from -50 to $90{ }^{\circ} \mathrm{C}$ change to a single-crystal-like KAI-type switching behavior at lower temperatures. ${ }^{283}$ Such change can be explained by the exponential decrease of domain wall motion speed with temperature. If the temperature is low enough, a new switching regime, where the domain wall motion limits the polarization reversal, becomes possible.

Switching in thin ferroelectric films may be affected by the lattice mismatch between film and substrate. In epitaxial PZT films, the strain induced by this mismatch is released through the formation of $a$ domains. ${ }^{284}$ These domains may strongly influence the polarization reversal. ${ }^{155,285}$ In the case of polycrystalline ferroelectric film capacitors, the nonuniform stress may provoke formation of anomalous microscopic polarization patterns. ${ }^{286}$

\section{Polarization imprint and retention}

The preferential polarization state induced in ferroelectric materials due to electrical or thermal treatment, which is known as polarization imprint, represents one of the main reliability issues for nonvolatile memories. The physical mechanisms proposed for the explanation of polarization imprint in ferroelectrics include dipole alignment, ${ }^{287}$ redistribution of charge carriers under the influence of the depolarization field, ${ }^{288}$ and charge injection through the interfacial layer. ${ }^{289,290}$ However, for ferroelectric thin films used for memory applications, only the latter scenario was found to be compatible with the totality of experimental observations. ${ }^{289}$ The concept employed in this scenario suggests that in the ferroelectric film the polarization decreases gradually within the nearby interfacial region, and this region is approximated by a low-dielectric-constant layer between the film and the interface. ${ }^{289,291}$ In the poled state, this layer is exposed to a very high electric field that provokes the charge injection, ${ }^{291}$ this injected charge captured in the interfacial layer being responsible for the voltage offset observed upon reversal of polarization in the ferroelectric material.

The offset of the hysteresis loop along the voltage axis is often used as a measure of imprint. This offset has been modeled numerically in Refs. 289, 292, and 293, assuming that the Pool-Frenkel conduction mechanism controls the charge injection. These calculations have yielded a logarithmic-type time dependence of the voltage offset and provided some justification for the logarithmic approximation of the time dependence of imprint used for evaluation of ferroelectric memories. However, the same group has later reported a more complicated behavior of imprint, characterized by a strongly nonlinear time dependence in a semilogarithmic scale. ${ }^{294}$ The problem of nonlinear behavior of imprint has been addressed in Ref. 290, where the charge injection provoking imprint was studied analytically, for different injection mechanisms. The major results of this work include an analytical description of nonlinear imprint and the limits of its logarithmic approximation, as well as the demonstration of a nonexponential temperature dependence of the logarithmic imprint rate. Based on these results, a model for imprint prediction has been proposed and verified using PZT film capacitors.

Polarization retention, which is the phenomenon of timedependent polarization loss in poled ferroelectric capacitors, represents another important reliability issue for ferroelectric memories. Retention can be typically characterized on the macroscopic level by pulse switching tests, where the amount of lost polarization in the poled state is represented as a function of time. ${ }^{295}$ On the microscopic level, retention has been investigated by time-resolved piezo-AFM mapping of domain arrangements in both SBT (Ref. 296) and PZT films. ${ }^{278}$ The origin of retention loss is most likely associated with the depolarization field or a built-in bias that provoke polarization back switching. ${ }^{296-298}$ In this context, it is natural to expect a close interconnection between the imprint and retention properties of the ferroelectric films, which is a topic requiring a more detailed investigation. A widely used method of improvement of the retention performance in ferroelectric capacitors is to modify the ferroelectric film composition by adding dopants. In particular, strong $\mathrm{Ca}$ and $\mathrm{Sr}$ doping has been shown to improve considerably the retention performance in PZT film capacitors used for nonvolatile memories. ${ }^{299}$

\section{Polarization fatigue in ferroelectric films}

In nonvolatile memory applications, ferroelectric film capacitors must deliver a stable switching charge, for a rather high number of switching cycles. For some applications, the specifications may require this number to be as high as $10^{13}$, which imposes severe reliability requirements on the memory capacitors. On the other hand, most ferroelectric materials exhibit degradation of switched polarization during the voltage cycling, known as polarization fatigue. Fatigue is regarded as an important reliability issue for PZT films and was studied intensively over the last decade in the context of nonvolatile memory applications. On the other hand, SBT films, which are also considered as candidates for nonvolatile 
memories, are known as "fatigue-free,"300-302 except for some particular conditions where fatigue has been observed. $^{303}$

A number of physical models for the polarization fatigue typically analyze the effect of electrical stress resulting either in a reduction of the electric field seen by the ferroelectric film or in the inhibition of the switching process. The first scenario suggests that a passive dielectric layer developing in the capacitor due to the electrical cycling provokes a decrease of the effective electric field. ${ }^{304}$ According to the second scenario, the switching is blocked due to the pinning of domain wall motion ${ }^{305,306}$ or due to the inhibition of the centers of opposite domain nucleation (domain seeds). ${ }^{136,307} \mathrm{~A}$ detailed critical review of different mechanisms of polarization fatigue in ferroelectric thin films presented in Ref. 136 suggests that the latter mechanism is probably more important for PZT films used for nonvolatile memories. A microscopic evidence in favor of the domain seed inhibition mechanism was obtained by piezo-SPM study, showing a partition of the fatigued films into large single-domain frozen areas. $^{280}$

Analyzing the microscopic origin of the charge that provokes domain pinning or domain seed inhibition, most authors evoke either oxygen vacancy migration ${ }^{308,309}$ or electron/hole injection from electrodes, ${ }^{136}$ driven by a very high electric field appearing during the polarization reversal in the nearby interfacial region.

Polarization fatigue in PZT films can be reduced considerably or even eliminated by using oxide electrodes such as $\mathrm{IrO}_{2}$ (Ref. 310) or $\mathrm{SrRuO}_{3}{ }^{311}$ Compared to the classical configuration with a ferroelectric film sandwiched between two Pt electrodes, the fatigue performance may be improved dramatically by using only one oxide top electrode. ${ }^{312}$ The proposed explanations of the positive impact of oxide electrodes on the fatigue performance vary depending on the physical model of fatigue. In the context of the model suggesting domain wall pinning by oxygen vacancies, the oxide electrode is considered as an oxygen vacancy sink. ${ }^{313}$ Alternatively, assuming domain seed inhibition due to the charge injected from the electrodes, the fatigue improvement was attributed to the increase of local conduction in the nearby electrode area that enhances the relaxation of injected charge. $^{312}$

Recent progress in integrated ferroelectric capacitor fabrication for nonvolatile memories resulted in virtually fatigue-free behavior of both PZT (Ref. 88) and SBT-based $^{302}$ capacitors up to $10^{13}$ switching cycles. As for PZT films with Pt electrodes, the use of dopants was shown to be efficient for fatigue performance improvement. ${ }^{314,315}$

\section{B. Nanoscale probing of ferroelectrics}

As the many possibilities of manufacturing ferroelectric thin films with tailored properties (using, for instance, atomic layer or molecular beam epitaxy methods) are currently growing immensely, the demand of the development and application of tools that are able to probe the physicochemical material parameters on the very local scale or even to ma- nipulate them becomes prominent. The requests upon such local measurement techniques are very demanding

- The technique should be able to laterally address nanoscale areas with a resolution much below the $100 \mathrm{~nm}$ scale, a boundary somehow artificially known as the "magic" limit separating the classical world of physics from nanotechnology.

- The method should equally reveal three-dimensional in-depth information about a ferroelectric film or ferroelectric structure.

- The tool, furthermore, should work noninvasively and probe the local physicochemical material properties without perturbing them, while, at the same time revealing the real quantitative local features of the system.

- Additionally, the method should be fast and applicable universally to any kind of structure that is being tested.

For the last 15 years, researchers have been applying scanning probe microscopy (SPM) to the investigation of ferroelectric and ferroelastic materials. While scanning tunneling microscopy (STM) could be applied only rarely, and due to conductivity only to stoichiometrically reduced or oxidized ferroelectrics, both scanning force (SFM) and scanning near field optical microscopy (SNOM) based methodologies have been intensively and successfully employed in order to satisfy the above requests. One should mention, though, that for SFM, ${ }^{316}$ the original setup by probing the local interaction force between an atomically sharp tip and the first or second near-surface atomic layer of the ferroelectric sample has rarely succeeded, and mostly for samples kept in vacuum. ${ }^{317}$ No example of an atomically resolved sample surface of a ferroelectric film has been reported to date. However, what researchers managed to develop are two elegant modes of coupling the SFM tip to both internal and external electric fields in such polar samples. Since ferroelectrics are piezoelectric, the fractional contribution of any nanoscale volume unit buried within the bulk of the ferroelectric or ferroelectric film system will contribute to a measurable signal through the three-dimensional piezoelectric tensor, provided we are able to address a sufficiently large electric field to that local inner volume fraction and being able to deduce its response and confound it from any background. This idea leads to the development of piezoresponse force microscopy (PFM) where the local SFM tip sits in contact with the ferroelectric sample surface and provides the local electric field for probing. Hence, PFM is able to literally look into the sample to a depth of approximately $100 \mathrm{~nm},{ }^{318}$ a value that is mostly governed by the local conductive properties of the ferroelectric sample (note that the electric field spherically emerging from the SFM tip in principle would penetrate ad infinitum into a perfect dielectric). The second SFM-based methodology that is suitable to address ferroelectric systems is probing the external electric field through noncontact SFM methods, such as Kelvin-probe force microscopy (KPFM). ${ }^{319}$ Since all the internal charges and electric field distributions within the sample give rise to a net surface charge density, the idea behind KPFM is probing the externally arising electric field and thence deducing the local ferroelectric proper- 


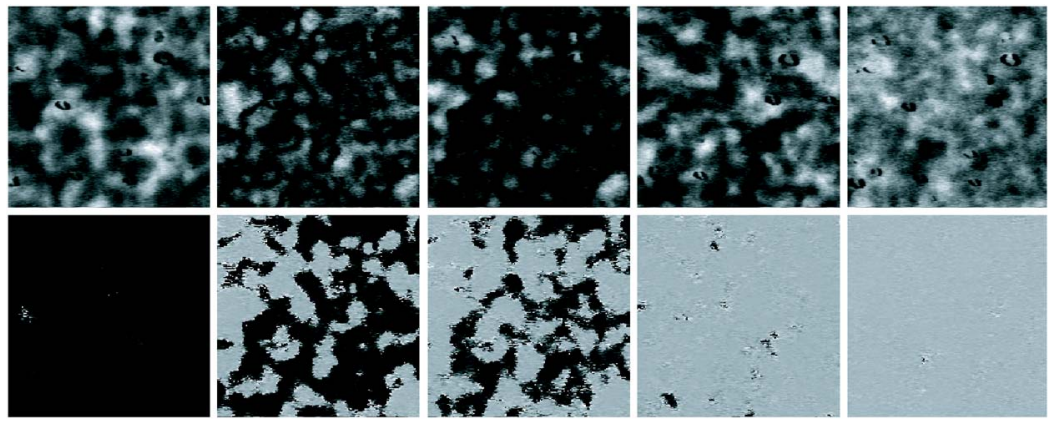

FIG. 33. Amplitude (upper) and phase (lower) evolution as a function of pulse field: $-48,-51,-54,-57$, and $-60 \mathrm{kV} / \mathrm{cm}$ pulses (from left to right images). $\langle 111\rangle \mathrm{Pb}\left(\mathrm{Zr}_{0.45} \mathrm{Ti}_{0.55}\right) \mathrm{O}_{3}$ film, cited from Ref. 326 .

ties. This method is noninvasive and provides quantitative data.

SNOM (Ref. 320) fits into this approach even more elegantly, since optical fields mostly do not suffer from absorption in such dielectric media or may be tuned easily into a more suitable spectral range. Like PFM, SNOM provides an electric field (at optical frequencies) that penetrates into the ferroelectric sample. Optical absorption, reflection, transmission, and even higher-order modes such as second- and thirdharmonic generations are possible contrast mechanisms that have been investigated and that may be further exploited. Note that practically all ferroelectrics are birefringent, as witnessed by the huge interest in these materials for optical light conversion in lasers and detectors. In contrast to PFM, however, it is the components of the electro-optical tensor rather than the piezoelectric tensor that are of interest to be probed here.

\section{Nanoscale ferroelectric domain imaging by piezoelectric force microscopy}

Piezoresponse force microscopy (PFM) was proposed by Franke and Weihnacht ${ }^{321}$ in 1995 and was developed by many research groups to become a standard tool for ferroelectric domain imaging.

Although the debate on the detection mechanism has not yet been finally settled, Kalinin and Bonnell ${ }^{322}$ and Harnagea and Pignolet ${ }^{323}$ have provided quite a complete picture.

PFM was initially aimed at probing the out-of-plane polarization vector ( $c$ axis) through a conductive probe, which acts as a movable electrode. The original PFM mode, which uses the tip as a movable top electrode and the bare ferroelectric film with bottom electrode as the medium, will be called local perturbation and detection (LPD) mode, because the electric field is highly localized underneath the tip and the tip detects locally the film movement. As the experience accumulated, there was a constant need to investigate the full three-dimensional vectors of polarization. Several groups showed that in-plane piezoelectric modes could be investigated using lateral force microscopy, which together with the normal mode could construct the polarization vector map. ${ }^{324,325}$

Colla et $a l .{ }^{280}$ aimed at identifying the direct correlation between the polarization hysteresis measured with a capacitor structure and the domain configuration. They placed the tip over the top electrode of the capacitor to probe the domain activities through the electrode. In this way, they were able to discover that locally frozen domains were responsible for the decrease of switchable polarization during fatigue. This method is named macroscopic perturbation, local detection (MPLD) mode, because the electric field between the bottom and top electrodes excites the whole film, while the tip only detects the local vibration of the film underneath the tip.

Setter and co-workers ${ }^{274,326}$ extended this work to explore the domain evolution during polarization switching with its corresponding macroscopic hysteresis curve, to find out that forward domain growth is the rate-limiting mechanism in $\langle 111\rangle$ oriented PZT thin films.

Moreover, it was found that the cantilever sample interactions were responsible for noise in the PFM measurement, and proposed that a high-aspect ratio tip or stiff cantilever be used to minimize the interactions. ${ }^{327}$ The latter suggestion aligns well with the later finding of a strong indentation mode, where the stiff cantilever would guarantee that most of the signal comes from the piezoelectric displacement underneath the tip. ${ }^{322}$

The former suggestion was widely accepted, so that most of the groups which conduct PFM measurements now use tips with a height greater than $10 \mu \mathrm{m}$. The other method was to shield the cantilever with top electrode, which is the same method used by Colla et al. ${ }^{280}$

Application of PFM. PFM is used for the study of three main issues: polarization switching and fatigue, local switching and domain stability, and three-dimensional analysis of the polarization vector.

a. Polarization switching and fatigue. Polarization switching and its degradation as a function of time or switching cycle pose a serious threat to the various commercial applications of FE materials, such as FRAMs, inkjet printer heads, laser projection units, and scanners. It is therefore important to identify their underlying mechanism, as discussed in detail in Sec. IV A.

PFM has opened the way to complement the missing link between macroscopic and microscopic manifestations of polarization switching by enabling us to image the polarization vector at nanoscale. Setter and co-workers provided some examples of linking the macroscopic polarization hysteresis loop to the domain evolution. ${ }^{274,326}$ Figure 33 shows the domain images as a function of pulse voltage applied between the top and bottom electrodes. The amplitude and phase images provide information on the polarization direction and its amplitude as a function of position, which constructs the full domain map along the $x, y$, and $z$ axes. Using PFM, Colla et al. found that the suppression of the remnant 


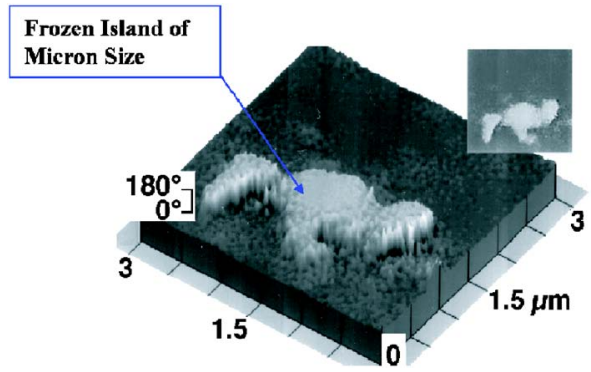

FIG. 34. Frozen island (bright) in a fatigued PZT sample $\left(10^{5}\right)$ showing preferential direction. The dark region can be fully switched. Cited from Ref. 280.

polarization occurs region by region, the typical size of the initially frozen regions being of the same order of magnitude as that of the grains. Figure 34 shows the shape and size of the frozen domain, which has lost the ability to switch but still contributes to the piezoelectric displacement. ${ }^{280}$

b. Local switching and domain stability. In the LPD mode, one can apply voltage through the tip to locally modify the domain structure. This idea led to the development of the probe storage concept based on PFM, as explained in detail by Shin. ${ }^{328}$

Applying a switching voltage to the tip results in local polarization switching, which is used as a writing procedure of bit information. This information can be read by extracting the phase or piezoresponse signal. ${ }^{329,330}$

In such applications, one of the main concerns is the thermal stability of written domains, which was studied by Woo et al. ${ }^{331}$ Figure 35 shows the experimental results that fully penetrated domains show better thermal stability than partially penetrated ones.

PFM has also been used to provide detailed local information on static domain wall configurations that are presumed to result from the interaction of the domain wall with pinning centers. These configurations have been analyzed in an effort to understand how domain wall creep takes place for ferroelectric thin films. ${ }^{128,332}$

Another example of local manipulation of domains is nanolithography that uses the PFM as a tool to locally switch domains and utilizes the domain dependent photochemical reactivity of metallic species to form nanoscale line patterns of metals. ${ }^{333}$

c. $3 D$ configuration of domains. Several groups ${ }^{324,325}$ have reported construction of the full three-dimensional polarization vector as a function of position on the surface plane. This technique enables one to understand the role of $a$ domains during polarization switching.

Another interesting method to obtain three-dimensional information is the domain mapping as a function of thickness. Hong et al. tried the same method of acquiring necessary depth information by etching the surface layer as in Auger electron spectroscopy (AES) or x-ray photoelectron spectroscopy (XPS) measurements. ${ }^{334}$ Figure 36 shows the amplitude and phase images of PFM before and after surface etching, where the thickness of the etched layer was about $500 \mathrm{~nm}$.

\section{Nanoscale probing of ferroelectrics by scanning near-field optical microscopy and related optical methods}

The activities concerning nano-optical approaches aimed at ferroelectric systems have been performed only with respect to the reading or characterization of ferroelectrics and domain structures, i.e., their analysis by means of confocal optical microscopy ${ }^{335}$ or linear and nonlinear SNOMs. Zayats and Smolyaninov ${ }^{336}$ recently reviewed the possibility of linear and nonlinear optical imaging with aperture and apertureless SNOMs (Refs. 337 and 338) for various materials, including ferroelectrics. Although the aperture SNOM
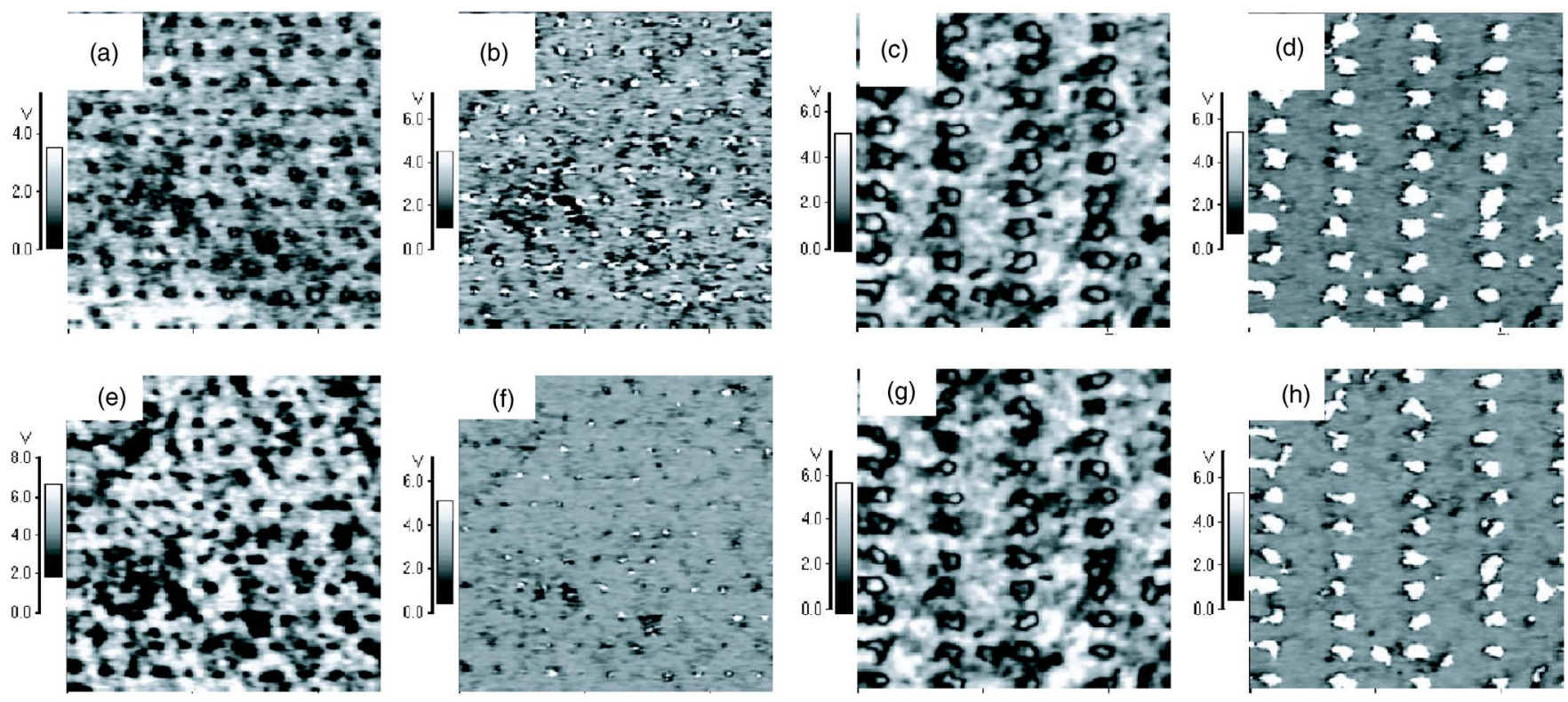

FIG. 35. Amplitude [(a), (c), (e), and (g)] and phase [(b), (d), (f), and (h)] images of a poled domain array with partially [(a), (b), (e), and (f)] and fully penetrating $\left[(\mathrm{c}),(\mathrm{d}),(\mathrm{g})\right.$, and (h)] domain configuration at room temperature $[(\mathrm{a})-(\mathrm{d})]$ and after heat treated at $100{ }^{\circ} \mathrm{C}$ for $30 \mathrm{~min}[(\mathrm{e})-(\mathrm{h})]$. Cited from Ref. 331. 

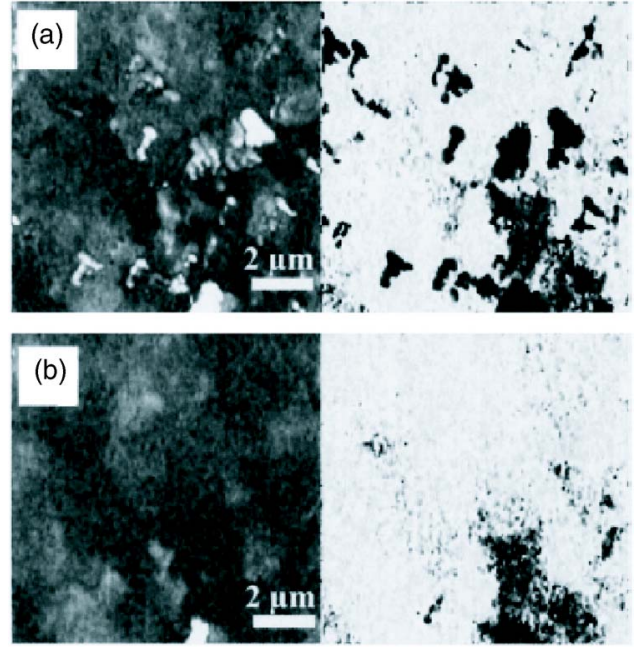

FIG. 36. Amplitude (left) and phase (right) images of a ferroelectric sample after (a) first and (b) second etching sequence. Cited from Ref. 334.

(a-SNOM) method allows higher-order modes, such as the second-harmonic ( $\mathrm{SH}$ ) signal, to be collected elucidating, for instance, strain effects in $\mathrm{BaTiO}_{3},{ }^{339}$ surface effects in PZT samples, ${ }^{340}$ or periodically poled $\mathrm{LiNbO}_{3}$ crystals, ${ }^{341}$ the resolution generally lies around $\sim 100 \mathrm{~nm}$ (Ref. 342) without much hope to go beyond this limit. Experiments on the dynamical behavior of domain walls were reported in Ref. 343, where the authors tracked the pinning and motion of a single $180^{\circ}$ domain wall in ferroelectric $\mathrm{LiTaO}_{3}$ under a uniform applied electric field. The optical change in the birefringent domain wall with applied field was attributed to the secondorder electro-optic and piezo-optic coefficients. Recent SNOM experiments by our own group showed that, in fact, the different components of the electro-optical tensor in ferroelectrics may be probed elegantly. ${ }^{344}$ A quantitative understanding is possible, provided that the electrical field distribution is known or modeled. ${ }^{345}$ Furthermore, Hubert et al. ${ }^{346}$ used this electro-optical coupling to perform time resolved measurements of the domain formation, achieving a resolution on the nanosecond time scale.

The difficulties in applying aperture SNOM to ferroelectrics are manifold.

- First, the lateral resolution should be improved down to a $10 \mathrm{~nm}$ spot size. With the standard setup of a-SNOM using a tapered optical fiber that carries a metallic aperture, any fabrication method employed so far can only provide aperture diameters down to $\sim 50 \mathrm{~nm}$. Due to material issues, this boundary cannot be crossed, which implies a limitation for future applications of the a-SNOM concept. A solution to this problem is using the so-called apertureless or scattering SNOM (s-SNOM). Here the SFM tip itself or any attached nanoparticle acts as the local antenna, focusing light to a tiny spot and confining the information to the particle diameter only. This approach offers a variety of possibilities, for instance, using near-field antennas that are specifically responding in the interesting frequency range of FTFs, i.e., in the infrared or near infrared, allowing soft modes and vibronic levels to be tracked locally. The investigation of polarons in domain walls, etc., is also of primary interest.

- Second, the light intensity at such small areas is of the order of femtowatts and needs another near-field scheme both for launching and detecting optical waves. Hence, when replacing a-SNOM by s-SNOM, the signal intensity already increases by two to three orders of magnitude. Moreover, the tip antenna structure may be fabricated in such a way that it optically resonates exactly at the same frequency as the ferroelectric sample of interest does. Hence, both the tip and sample oscillators are matched giving rise to a huge resonant signal that is easily measurable. Note that for metallic tip antennas, for instance, surface plasmon resonances in the visible optical regime can be used ${ }^{347}$ while dielectric samples show their resonances in the infrared. ${ }^{348}$ A further efficient way of increasing the local optical intensity is using ultrashort laser pulses for probing. Such pulses show two further advantages, in that (a) the FE material under investigation will suffer less when probed on the femtosecond time scale, while (b) an ultrashort laser pulse per se provides a broad or white frequency spectrum.

- Third, the local anisotropy in ferroelectrics arises equally from both the birefringence between differently oriented domains ${ }^{349}$ and from local strain either at domain walls or between the substrate and the FE material. ${ }^{350}$ Note that beyond ferroelastic domain walls ${ }^{351}$ also $180^{\circ}$ domain walls in FEs (Refs. 352 and 353) give rise to appreciable optical contrast effects that are of importance to be probed locally. a-SNOM per se is limited again in that the local (metallic) tip aperture severely disturbs the local electromagnetic field distribution, making investigations of the local anisotropy by simple linear a-SNOM practically impossible. s-SNOM, on the contrary, provides a clear way out of this. ${ }^{349}$

The future of near-field optical investigations in ferroelectric films using scattering-SNOM based methodologies is indeed very promising. Not only will this technology be of valuable help when tracking and probing the different material properties in ferroelectrics or ferroelectric films or even addressing dynamical issues, for instance, when switching ferroelectric devices, but also the nano-optical approach supports the capability of even writing and manipulating domains or domain walls by optical means on the nanometer scale. Hence, in principle, a fully optically operated memory device will become possible.

\section{Extrinsic and intrinsic nonlinearities of piezoelectric properties of ferroelectric thin films}

In the following, the dynamic and static piezoelectric nonlinearities in $\mathrm{Pb}(\mathrm{Zr}, \mathrm{Ti}) \mathrm{O}_{3}$ thin films are discussed in terms of film composition, crystallographic orientation, and microstructure.

A significant part of the piezoelectric response of bulk ferroelectric materials may originate from the displacement of domain walls. ${ }^{354,355}$ For most applications, this so-called 
extrinsic contribution to the piezoelectric properties is undesired because it is nonlinear, hysteretic, and dispersive, even at weak-to-moderate driving fields. ${ }^{356-360}$ The domain wall contributions depend on many parameters, including the composition, crystal structure, dopants or impurity concentration, and microstructure of the material. ${ }^{361}$

For typical ferroelectric film thicknesses ranging from $100 \mathrm{~nm}$ to $1 \mu \mathrm{m}$, a driving voltage of only $0.1 \mathrm{~V}$ translates into large fields between $1 \mathrm{MV} / \mathrm{m}$ and $100 \mathrm{kV} / \mathrm{m}$. In bulk materials, these fields are well within the region where the nonlinearity, hysteresis, and dispersion are non-negligible. On the other hand, it is known that the so-called intrinsic response (response of an individual domain) in thin films is strongly affected by the misfit strains and substrate clamping. ${ }^{190,362}$ Considering boundary conditions specific to thin films (clamping by the substrate and misfit strain), their small thickness and grain size, and effects related to special preparation procedures (low processing temperatures, sol-gel technique, sputtering, pulsed laser deposition, etc.), the question arises of whether the domain wall contribution in thin films is considerably modified with respect to that in bulk materials. The interest in the domain wall contribution in thin films also stems from the possibility of growing films with controlled texture and crystal orientation, allowing to engineer the piezoelectric properties by controlling both the intrinsic and extrinsic effects. For example, in poled films of the same composition but with different crystal orientations the domain wall configuration is not the same, and both intrinsic and extrinsic contributions to the piezoelectric response will be different.

In this section, recent results on the domain wall contribution to the piezoelectric response of PZT thin films are reviewed. First, evidence is presented of a non-negligible domain wall contribution to the piezoelectric properties in ferroelectric thin films, followed by a brief discussion of compositional, crystal orientation and microstructural effects on the piezoelectric nonlinearity. Competing effects of intrinsic and extrinsic piezoelectric nonlinearities are also discussed.

\section{Evidence of domain wall contribution to the piezoelectric response of thin films}

As in bulk materials, there is only indirect evidence of the contribution of domain wall displacement to the piezoelectric response of thin films at subcoercive fields. By subcoercive fields here we mean fields below the coercive field, i.e., the field at which the polarization of a macroscopic sample switches under usual experimental conditions. ${ }^{361}$ An example of such indirect evidence is given in Fig. 37, which shows longitudinal strain-electric field hystereses for different amplitudes of the driving electric field for a $0.9 \mu \mathrm{m}$ thick $\mathrm{Pb}\left(\mathrm{Zr}_{0.60} \mathrm{Ti}_{0.40}\right) \mathrm{O}_{3}$ thin film with $[111]_{c}$ orientation. The index $c$ designates that the crystallographic orientation is given with respect to the cubic cell. Such nonlinear hystereses, with an accompanying field dependence of the piezoelectric coefficient, ${ }^{363}$ are typical of systems in which domain walls move in a medium with randomly distributed pinning centers. ${ }^{356}$

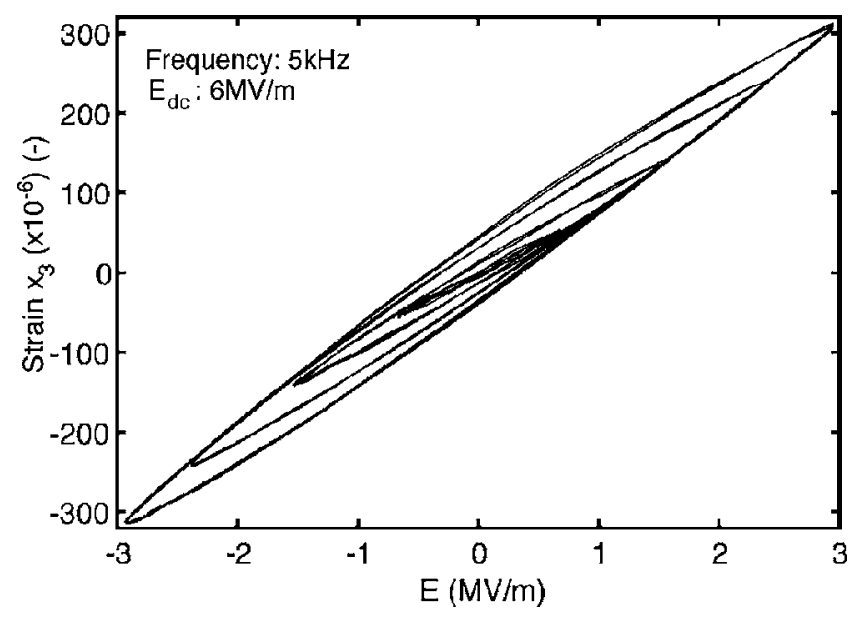

FIG. 37. Nonlinear strain-electric field hystereses for a $[111]_{c}$ oriented $0.9 \mu \mathrm{m}$ thick sol-gel derived $\mathrm{Pb}\left(\mathrm{Zr}_{0.6} \mathrm{Ti}_{0.4}\right) \mathrm{O}_{3}$ thin film. Measurements were made under unipolar conditions with dc electric bias field of $6 \mathrm{MV} / \mathrm{m}$. The loops are centered numerically. For details see Ref. 447.

The analytical expression for the strain-field hysteresis and the field dependence of the piezoelectric coefficient is given by the Rayleigh law, ${ }^{363-365}$ which, in a more general case, can be modified as follows:

$$
\begin{aligned}
& x(E)=\left[d_{i}+\alpha^{\prime}\left(E_{0}\right) E_{0}\right] E \pm \frac{\alpha^{\prime}\left(E_{0}\right)}{2}\left(E_{0}^{2}-E^{2}\right), \\
& d=d_{i}+\alpha^{\prime}\left(E_{0}\right) E_{0},
\end{aligned}
$$

where $x$ is the strain, $E=E_{0} \sin (\omega t)$ the electric field, $d_{i}$ the reversible piezoelectric coefficient, and $\alpha^{\prime}=\alpha+\beta E_{0}+\gamma E_{0}^{2}$ $+\cdots$ the field-dependent Rayleigh parameter. The classical Rayleigh law, which is valid for a perfectly random distribution of pinning centers, ${ }^{366}$ is obtained from Eq. (6) by taking $\beta=\gamma=\cdots=0$. The modified expression [Eq. (6)] takes into account any possible deviation from ideal Rayleigh behavior while keeping the essential link between the hysteresis and nonlinearity. ${ }^{367}$

In comparison with bulk materials, the domain wall contribution to the piezoelectric effect in thin films is, in general, found to be restricted and strongly dependent on film thickness, microstructure, and crystal orientation. ${ }^{368,369}$ The main reason for the weaker domain wall contribution in thin films is that films are clamped by the substrate. It is usually considered that only displacement of ferroelastic (non- $180^{\circ}$ ) domain walls can contribute to the piezoelectric effect. Since displacement of ferroelastic domain walls changes the shape of the film, their movement is expected to be restricted in films due to clamping by the substrate. A detailed analysis of microstructural and structural effects (grain size, film thickness, and texture) on the domain wall contribution to the piezoelectric effect can be found in Ref. 370 . The following sections will briefly review the most important results.

By using a simple model based on the Rayleigh law, it has been recently demonstrated that, in some cases, the displacement of purely ferroelectric (nonferroelastic) $180^{\circ}$ domain walls can contribute to the piezoelectric strain. ${ }^{369}$ The predictions of this model are in good agreement with experimental data. 


\section{Film orientation and piezoelectric nonlinearity in $P Z T$}

Since the discovery of the large piezoelectric response in relaxor-ferroelectric single crystals along nonpolar directions, ${ }^{371}$ it was realized that analogous enhancement of the piezoelectric response should be a common characteristic of perovskite materials. ${ }^{372}$ In PZT films, in particular, this possibility was of interest since single crystals are not available and the effect can be verified only in epitaxial or highly oriented films. In addition, this intrinsic anisotropy allows engineering of the piezoelectric properties, as shown in Fig. 38 for PZT films with rhombohedral $\left[\mathrm{Pb}\left(\mathrm{Zr}_{0.60} \mathrm{Ti}_{0.40}\right) \mathrm{O}_{3}\right]$, tetragonal $\left[\mathrm{Pb}\left(\mathrm{Zr}_{0.45} \mathrm{Ti}_{0.55}\right) \mathrm{O}_{3}\right]$, and morphotropic phase boundary $\left[\mathrm{Pb}\left(\mathrm{Zr}_{0.53} \mathrm{Ti}_{0.47}\right) \mathrm{O}_{3}\right]$ compositions. The concept was successfully used to engineer films with a large $e_{31}$ piezoelectric coefficient, of interest for microelectromechanical applications. $^{26}$

In PZT films with the rhombohedral structure, Fig. 38(c), the polarization is oriented along the cubic $[111]_{c}$ direction, whereas the largest response is measured along the $[001]_{c}$ axis. In addition, rhombohedral films poled along the $[001]_{c}$ axis are supposed to possess a domain configuration in which all domain states are ideally equivalent. ${ }^{371}$ If an electric field is applied along the $[001]_{c}$ axis, the contribution of the domain wall displacement to the piezoelectric strain is expected to be small. ${ }^{373}$ This is indeed confirmed experimentally by the reduced piezoelectric hysteresis and nonlinearity in $[001]_{c}$ films with respect to $[111]_{c}$ oriented films with ferroelastically active walls, cf. Fig. 38 (c) ${ }^{373}$ Ideally, the displacement of domain walls in $[001]_{c}$ oriented films should not give a contribution to the piezoelectric effect and the $d_{33}$ coefficient should be field independent. The origin of the experimentally observed field dependence of $d_{33}$ could then lie with the nonperfect film orientation and, more importantly, could indicate that the domain configuration is not as simple as usually thought, or that other (Ref. 369) or presently unknown processes contribute to the nonlinearity.

Analogous behavior is observed in films with the tetragonal composition, Fig. 38(a), where the polar direction lies now along the $[001]_{c}$ axis, and the smallest hysteresis and nonlinearity are observed along the $[111]_{c}$ direction. Note that in these tetragonal films the maximum piezoelectric response does not appear along the nonpolar $[111]_{c}$ direction, Fig. 38(a). This experimental result is consistent with the predictions of the Landau-Ginzburg-Devonshire theory. ${ }^{374}$

Films with the composition in the morphotropic phase boundary region exhibit similar nonlinearity regardless of orientation, reflecting the simultaneous presence of a mixture of tetragonal and rhombohedral phases in composition, cf. Fig. 38(b). In summary, the good agreement of the experimental dynamic nonlinear piezoelectric behavior in these films with that expected from the crystal structure and domain configuration of each oriented film gives additional evidence of a domain wall contribution to the piezoelectric response in thin films.

\section{Domain wall contribution and film microstructure}

A detailed analysis of the relation between the microstructure and the piezoelectric and dielectric properties of

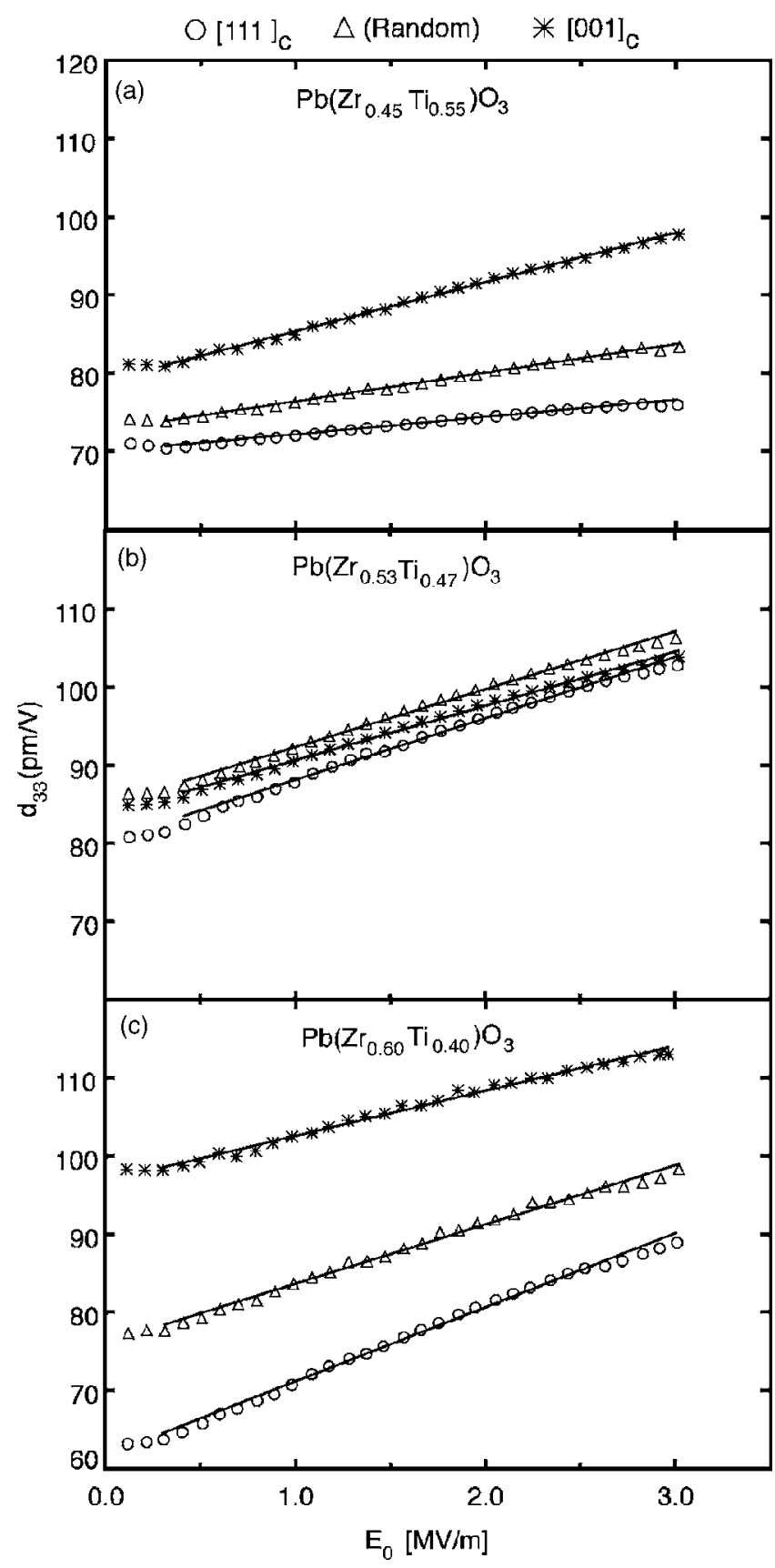

FIG. 38. Dependence of the longitudinal converse piezoelectric coefficient $d_{33}$ on the amplitude $E_{0}$ of the sinusoidal driving electric field for sol-gel derived PZT films with (a) tetragonal, (b) mixed tetragonal-rhombohedral, and (c) rhombohedral structures. All films have thickness of about $0.9 \mu \mathrm{m}$. The measurements were made using the laser interferometer, at $5 \mathrm{kHz}$ and at a bias field of $6 \mathrm{MV} / \mathrm{m}$. For details see Ref. 373. The lines through the data points represent fits with the Rayleigh law [Eq. (6) and $\beta=\gamma=0$ ].

PZT films has been carried out by Xu et al., ${ }^{370}$ enabling them to evaluate the contribution of domain wall displacement to the electromechanical properties of PZT films. The conclusion of this study was that in thinner films and films with a small grain size, the motion of ferroelastic non- $180^{\circ}$ domain walls is reduced; only films with a relatively large thickness $(>5 \mu \mathrm{m})$ and grain size show a domain wall contribution comparable to that of bulk materials. As already mentioned in Sec. IV C 1, this result is consistent with a stronger clamping by the substrate in thinner films, limiting the motion of 
ferroelastic domain walls. A recent study of La-doped lead titanate films, ${ }^{368}$ however, shows that films as thin as $340 \mathrm{~nm}$ with an average grain size between 100 and $300 \mathrm{~nm}$ still exhibit strong piezoelectric nonlinearity. This suggests that the processes that control domain wall pinning and clamping are complex and influenced by many parameters, some of which are presently poorly understood.

\section{Intrinsic nonlinearity and domain wall contribution}

Besides the extrinsic nonlinearity that has its origin in the displacement of domain walls, piezoelectric properties of ferroelectric films exhibit a contribution from the intrinsic, lattice nonlinearity. This nonlinearity can be readily estimated by the thermodynamic Landau-Ginzburg-Devonshire (LGD) theory, which in the case of thin films must include terms related to the strain induced by the mismatch in lattice parameter between the film and the substrate (so-called misfit strain). Chen et al. ${ }^{362}$ have calculated $d_{33}$ as a function of the electric bias field, $E_{\mathrm{dc}}$, in several PZT compositions, and have shown that the piezoelectric coefficient decreases with increasing bias field. Interestingly, they did not examine nonlinearity at negative bias fields, which are usually also employed during measurements of $d_{33}-E_{\mathrm{dc}}$ loops. We shall now briefly discuss $d_{33}-E_{\mathrm{dc}}$ loops in tetragonal PZT films (cf. Fig. 39) and demonstrate competing effects of domain wall processes and lattice nonlinearity. For simplicity, we shall discuss only the part of the loops where $d_{33}>0$. The sign of $d_{33}$ is explained in the figure caption.

Experimental $d_{33}-E_{\mathrm{dc}}$ loops for three orientations of tetragonal $0.9 \mu \mathrm{m}$ thick $\mathrm{Pb}\left(\mathrm{Zr}_{0.45} \mathrm{Ti}_{0.55}\right) \mathrm{O}_{3}$ thin films are shown in Fig. 39(a). Loops for $[001]_{c}$ and $[111]_{c}$ oriented films are qualitatively different and the loop for the film with random orientation shows characteristics between those of two oriented films. In the $[001]_{c}$ oriented film, when the field has reached its maximum and begins to descend, $d_{33}$ first slightly increases and then begins to decrease at fields well above $E_{\mathrm{dc}}=0$. In the $[111]_{c}$ film, $d_{33}$ increases with decreasing field and begins decreasing only when $E_{\mathrm{dc}}<0$. To understand this difference, we next analyze the expected intrinsic behavior of $d_{33}-E_{\mathrm{dc}}$ loops.

Figure 39(b) shows the value of $d_{33}\left(E_{\mathrm{dc}}\right)$ calculated using the LGD theory for a $[001]_{c}$ oriented $\mathrm{Pb}\left(\mathrm{Zr}_{0.40} \mathrm{Ti}_{0.60}\right) \mathrm{O}_{3}$ crystal. The data needed for phenomenological calculations are taken from Ref. 375. The $\mathrm{Pb}\left(\mathrm{Zr}_{0.40} \mathrm{Ti}_{0.60}\right) \mathrm{O}_{3}$ composition is chosen as it is nearest to $\mathrm{Pb}\left(\mathrm{Zr}_{0.45} \mathrm{Ti}_{0.55}\right) \mathrm{O}_{3}$, for which the values of the phenomenological coefficients are not available. The most interesting result is that $d_{33}$ increases with increasing antiparallel (negative) field. ${ }^{376} \mathrm{~A}$ qualitatively similar loop behavior, with different absolute values for the piezoelectric coefficient, is obtained along the $[111]_{c}$ axis (not shown in the figure). It is known that in ferroelectric materials the coercive field calculated from the thermodynamic theory is many times higher than the experimental coercive field. In Fig. 39(b) we choose, for the purposes of comparison only, a coercive field similar to the ones observed experimentally in $\mathrm{Pb}\left(\mathrm{Zr}_{0.45} \mathrm{ti}_{0.55}\right) \mathrm{O}_{3}$ films, cf. Fig. 39(a). In addition, as demonstrated by Chen et al., ${ }^{362}$ the value of $d_{33}$ is lower in a thin film than in a monodomain bulk crystal of the same material, due to substrate clamping

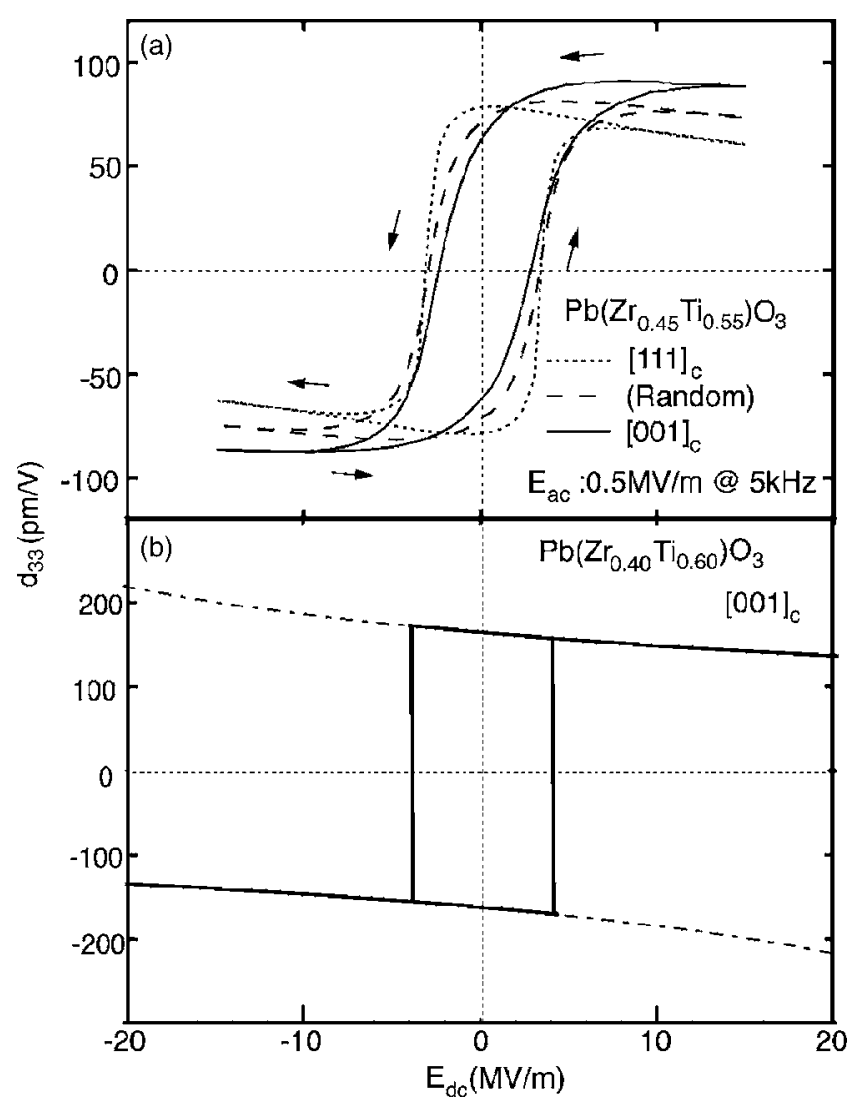

FIG. 39. (a) The experimental $d_{33}-E_{\mathrm{dc}}$ loops for different orientations of $\mathrm{Pb}\left(\mathrm{Zr}_{0.45} \mathrm{Ti}_{0.55}\right) \mathrm{O}_{3}$ films. (b) The $d_{33}-E_{\mathrm{dc}}$ loop calculated using the LandauGinzburg-Devonshire theory for $[001]_{c}$ oriented $\mathrm{Pb}\left(\mathrm{Zr}_{0.4} \mathrm{Ti}_{0.6}\right) \mathrm{O}_{3}$ tetragonal monodomain single crystals. The vertical solid lines in (b) indicate realistic coercive fields [cf. (a)], while the dashed-dotted and solid horizontal lines in (b) are the calculated values of $d_{33}\left(E_{\mathrm{dc}}\right)$. The arrows indicate the direction of loop rotation. The change in the sign of the piezoelectric coefficient indicates change in the phase angle of the piezoelectric strain.

and misfit strain. However, the general form of the $d_{33}-E_{\mathrm{dc}}$ dependence is qualitatively similar in films and crystals of the same composition. ${ }^{362}$

By comparing Figs. 39(a) and 39(b), it is clear that the $d_{33}-E_{\mathrm{dc}}$ loop of the $[111]_{c}$ oriented tetragonal film is reproduced by the shape of the theoretical loop much more accurately than the loop of the film with $[001]_{c}$ orientation. The reason for this is discussed below. Again, for simplicity, we shall consider only the part of the loops where $d_{33}>0$. The sign of $d_{33}$ is explained in the figure caption.

Tetragonal films contain ferroelectric $180^{\circ}$ and ferroelastic $90^{\circ}$ domain walls. These domains can contribute to the piezoelectric effect in two ways. First, the displacement of $90^{\circ}$ domain walls enhances the dynamically measured response. On the other hand, in tetragonal PZT their presence decreases the piezoelectric response of the films because of the intrinsic anisotropy of the crystal. ${ }^{374}$ Finally, the reorientation of $90^{\circ}$ domain walls can contribute to a more complete poling of the film. As $E_{\mathrm{dc}}$ increases, there are therefore three effects: (i) switching of $90^{\circ}$ walls, and enhanced $d_{33}$ via the anisotropy effect and a more complete poling; (ii) decreased $d_{33}$ because of the smaller contribution from the lower number of moving domain walls; and (iii) decreased response due to the intrinsic nonlinear effect. The form of the $d_{33}$ 
$-E_{\mathrm{dc}}$ loop will depend on the competing effects of these three contributions. In tetragonal films with $[111]_{c}$ orientation (the so-called $3 \mathrm{~T}$ domain structure ${ }^{377}$ ) the domain configuration is such that the motion of domain walls and domain switching does not change the shape of the film perpendicularly to the film surface $\left(d_{33}\right.$ effect). This is so because all possible $90^{\circ}$ domain walls have the same projection along the bias field direction: ideally, there should be no contribution of the moving domain walls to the dynamic $d_{33}$ and no change in the anisotropy effects if the $90^{\circ}$ domain walls switch or move. However, reversal by $180^{\circ}$ increases $d_{33}$, because of better film poling. Thus, only the intrinsic nonlinearity and poling should contribute to $d_{33}\left(E_{\mathrm{dc}}\right)$ in films with this orientation. By comparing the experimental and theoretical loops it is clear that for this orientation the intrinsic nonlinearity dominates at all fields from maximum to zero. When $E_{\mathrm{dc}}$ decreases below zero, $d_{33}$ decreases rapidly, probably because of domain reversal by $180^{\circ}$ and nucleation of oppositely oriented domains, which apparently occurs even at very small bias fields antiparallel to the polarization. Ideally, even at negative fields, $d_{33}$ should continue to increase with increasing antiparallel bias field, as indicated in Fig. 39(b). Such behavior, while not observed here, has been reported in some thin films ${ }^{378}$ and ceramics. ${ }^{379}$

In tetragonal films with $[001]_{c}$ orientation, the movement of $90^{\circ}$ domain walls changes the shape of the film both within the film plane and perpendicular to the film surface; thus, their displacement contributes to the longitudinal piezoelectric response. At large $E_{\mathrm{dc}}$ this contribution decreases because domains reorient along the field as the field increases and the number of domain walls decreases; on the other hand, the ensuing poling of the film enhances the piezoelectric effect. Finally, both effects compete with the intrinsic nonlinearity, which decreases the piezoelectric effect with increasing field parallel to polarization. From the shape of the $d_{33}$ loop for the film with $[001]_{c}$ orientation, it can be concluded that extrinsic effects dominate the $d_{33}-E_{\mathrm{dc}}$ loop: even at largest applied fields, domain wall reorientation (poling) is not complete. This poling process which enhances $d_{33}$ is comparable to the intrinsic nonlinearity that decreases $d_{33}$, making $d_{33}\left(E_{\mathrm{dc}}\right)$ approximately constant. At positive fields close to zero, $d_{33}$ starts to decrease rapidly. This effect is most likely due to the back switching of $90^{\circ}$ walls, whose previous removal from the equilibrium position by $E_{\mathrm{dc}}$ changes the shape of the film and is thus opposed by the stresses associated with substrate clamping. At $E_{\mathrm{dc}}<0,180^{\circ}$ domain reversal and nucleation of oppositely oriented domains are probably responsible for the decrease of $d_{33}$.

In rhombohedral films for which the dynamic piezoelectric nonlinearity is shown in Fig. 38, the $d_{33}-E_{\mathrm{dc}}$ loops (not shown in the figure) for all three orientations are dominated by the intrinsic nonlinearity at high bias fields. At low bias fields, the shape of the loops is controlled by the ferroelastic domain wall switching, regardless of the film orientation. It appears that in these films either the domain structure is not exactly as expected or additional mechanisms contribute to the static piezoelectric nonlinearity.

To sum up, piezoelectric nonlinearity in ferroelectric thin films has origins in both intrinsic (lattice) and extrinsic (do- main wall related) processes. Dynamic nonlinearity is dominated by the displacement of domain walls. Under static (dcbias) fields, the nonlinearity is a result of the balance between domain reorientation and intrinsic nonlinearity, which have opposite effects. Both contributions are strongly dependent on the crystallographic orientation of the films, allowing a significant degree of engineering of the piezoelectric properties. In some cases, additional, presently unidentified processes may contribute to the nonlinearity.

\section{Permittivity and loss in thin films}

The dielectric response of ferroelectric thin films is usually different from that of bulk ferroelectric materials. Typically, the permittivity of the films is lower; however, sometimes its temperature anomaly is shifted along the temperature axis with respect to that in the bulk material. In the latter case, for a given temperature, the permittivity in the film can be higher than in the bulk material. According to the literature, the loss tangent in the films is always higher than in their bulk counterparts. The question of the origin of the difference in the dielectric response between films and bulk materials has been intensively discussed in the literature. Several mechanisms have been proposed to explain this difference. For the permittivity, one mainly discusses the effect of mechanical stressing of the film by the substrate, depolarizing effects, and the poling effect of the ferroelectric/ electrode interface. For the loss tangent, the contributions due to charged defects and local polar regions, as well as the field-induced quasi-Debye contribution, are under consideration. In general, the understanding of the permittivity issues is much more developed than that of the loss issues. Below we will briefly outline the main points of these theoretical models and discuss their relevance to real experimental situations.

Mechanical stressing and clamping of the film by the substrate influence both the lattice and domain contributions to the permittivity. The lattice contribution affected by mechanical stressing and clamping has been comprehensively described in terms of the phenomenological Landau approach. ${ }^{380-383}$ It has been found that the mechanical effects modify the temperature of ferroelectric phase transitions, $T_{C}$, the corresponding Curie-Weiss temperature, $T_{0}$, and the Curie-Weiss constants, $C$. The important result is a linear dependence of $T_{0}$ on the misfit strain imposed by the substrate onto the films in the paraelectric state. Quantitative calculations have been performed for (001) and (111) films of multiaxial ferroelectrics of $\mathrm{BaTiO}_{3}$ type. In this case, the dielectric response of the material becomes anisotropic, so that one introduces different Curie-Weiss temperatures, $T_{\text {in }}$ and $T_{\text {out }}$, and different Curie-Weiss constants, $C_{\text {in }}$ and $C_{\text {out }}$, to describe the in-plane and out-of-plane components of the permittivity in the paraelectric phase, ${ }^{380,382}$

$$
C_{\text {in,out }}=\frac{C}{1-A \Delta \alpha},
$$


TABLE III. Parameters controlling the impact of the misfit strain on the temperature anomaly of dielectric permittivity of typical ferroelectric films. The experimental data used for the calculation (Ref. 450) are taken from Refs. 381, 443, and 444.

\begin{tabular}{|c|c|c|c|c|}
\hline & \multicolumn{2}{|c|}{ (001) } & \multicolumn{2}{|c|}{ (111) } \\
\hline & $A_{\text {in }}\left(10^{4} \mathrm{~K}\right)$ & $A_{\text {out }}\left(10^{4} \mathrm{~K}\right)$ & $A_{\text {in }}\left(10^{4} \mathrm{~K}\right)$ & $A_{\text {out }}\left(10^{4} \mathrm{~K}\right)$ \\
\hline $\mathrm{PbTiO}_{3}$ & 3 & -2.5 & 2.7 & 0.16 \\
\hline $\mathrm{BaTiO}_{3}$ & 3.6 & -4.7 & 2.3 & -0.32 \\
\hline $\mathrm{SrTiO}_{3}$ & 3 & -1.5 & 1.8 & 1.2 \\
\hline
\end{tabular}

$$
T_{\text {in,out }}=\frac{T_{0}+A\left(\varepsilon_{P_{0}}-\Delta \alpha T_{d}\right)}{1-A \Delta \alpha},
$$

where the corresponding value of $A_{\text {in }}$ and $A_{\text {out }}$ should be used for $A$, and $\varepsilon_{P_{0}}$ is the misfit strain at the deposition temperature $T_{d}$. The equation for $T_{\mathrm{in}, \text { out }}$ was obtained under the assumption that below $T_{d}$ no dislocation-assisted release of the misfit strain takes place; $\Delta \alpha$ is the difference between the thermal expansion coefficients of the substrate and the film. These relations have been written for the case where the misfit strain in the paraelectric phase and the in-plane thermal expansion of the substrate are isotropic. The parameters $A_{\text {in }}$ and $A_{\text {out }}$ are functions of the components of the electrostrictive and compliance tensors, $Q_{i j}$ and $s_{i j}$. For the (001) films,

$$
\begin{aligned}
& A_{\text {in }}=2 \varepsilon_{0} C \frac{Q_{11}+Q_{12}}{s_{11}+s_{12}}, \\
& A_{\text {out }}=2 \varepsilon_{0} C \frac{2 Q_{12}}{s_{11}+s_{12}},
\end{aligned}
$$

and, for (111) films, ${ }^{449}$

$$
\begin{aligned}
& A_{\text {in }}=2 \varepsilon_{0} C \frac{4\left(Q_{11}+2 Q_{12}+Q_{44} / 2\right)}{4 s_{11}+8 s_{12}+s_{44}}, \\
& A_{\text {out }}=2 \varepsilon_{0} C \frac{4\left(Q_{11}+2 Q_{12}-Q_{44}\right)}{4 s_{11}+8 s_{12}+s_{44}} .
\end{aligned}
$$

The values of the parameters $A_{\text {in }}$ and $A_{\text {out }}$ for some perovskite ferroelectrics are listed in Table III.

The effects predicted by Eq. (7) may be appreciable. For realistic parameters of ferroelectric thin films, one expects shifts of $T_{0}$ up to a few hundred Kelvin and up to a $30 \%$ modification of the Curie-Weiss constant.

The predictions of the theoretical approach reviewed above agree with the experimental observations in perovskite thin films. A few examples are given below.

In the case of relatively thick films, the theory states that the variation of the Curie-Weiss temperature and that of the Curie-Weiss constant are controlled by the sign of $A \Delta \alpha$ and are correlated: a decrease of the former implies an increase of the latter, and vice versa. This kind of behavior was reported by Pertsev et al. ${ }^{380}$ for $\mathrm{BaTiO}_{3}$ and $\mathrm{SrTiO}_{3}$ films deposited onto $\mathrm{Si}$. The authors found the experimentally observed variation of $T_{\text {out }}$ and $C_{\text {out }}$ to be in reasonable agreement with the theory presented above. In the case of an ultrathin $(12 \mathrm{~nm})$ film of $\mathrm{BaTiO}_{3}$ deposited onto a compressive $\mathrm{SrTiO}_{3}$ substrate, a $500 \mathrm{~K}$ increase of the transition temperature was documented ${ }^{384}$ from the out-of-plane dielectric testing of the material. The value of $A_{\text {out }}$ corresponding to this situation, $4.7 \times 10^{4} \mathrm{~K}$, leads to an upward shift of the Curie-Weiss temperature by some $500 \mathrm{~K}$ for an in-plane strain of $1 \%$. This value is comparable to the data on the in-plane lattice constant of the $\mathrm{BaTiO}_{3}$ films reported in the same paper. A clear manifestation of the strain effects on the permittivity in films of $\mathrm{SrTiO}_{3}$ has been reported by Haeni et $a l .{ }^{208}$ They observed a strong enhancement of the permittivity in the case of a $\mathrm{DyScO}_{3}$ substrate (with positive misfit strain), and a strong reduction of the permittivity in the case of a $\left(\mathrm{LaAlO}_{3}\right)_{0.29}\left(\mathrm{SiAl}_{05} \mathrm{Ta}_{0.5} \mathrm{O}_{3}\right)_{0.71}$ substrate (with negative misfit strain). The observed variations of permittivity are compatible with the theoretical predictions, e.g., a $250 \mathrm{~K}$ increase of the "in-plane" Curie-Weiss temperature in the case of the $\mathrm{DyScO}_{3}$ substrate.

The modification of the Curie-Weiss anomaly discussed above fully covers the modification of the dielectric response of the films in the paraelectric phase. In the ferroelectric phase, additional effects become important.

If the ferroelectric state is single domain, then the additional role of mechanical stressing and clamping is mainly reduced to a modification of the parameters of the ferroelectric state (i.e., the value and direction of the spontaneous polarization). This will obviously influence the value of the lattice contribution to the permittivity in the ferroelectric state. As for the qualitative features of the dielectric response, it is worth mentioning the effect of strain-induced smearing of the dielectric anomaly. ${ }^{383}$ It has been shown, ${ }^{383}$ using the available thermodynamic parameters of $\mathrm{PbTiO}_{3}$, that in a (111) film of this material, one expects an essential modification of the temperature anomaly in the out-of-plane component of the permittivity, $\varepsilon_{33}$ : under tensile misfit strain in the paraelectric phase, a smeared anomaly is predicted, whereas under compressive misfit strain, $\varepsilon_{33}$ is predicted to exhibit a singular temperature anomaly typical of a second order phase transition. Thus, in this system, while in the bulk form a nonsingular but abrupt dielectric anomaly (the firstorder phase transition) occurs, in the (111) film the out-ofplane dielectric anomaly becomes singular or smeared depending on the sign of the misfit strain.

If the ferroelectric phase transition is accompanied by splitting into ferroelastic domains, which partially release the substrate-induced stress in the films, the appeared domain pattern contributes to the total permittivity of the films. There is an essential difference between this contribution and the domain contribution in a mechanically free bulk single crystal ferroelectric. In the bulk material, the domain contribution to the permittivity is controlled by defect pinning of the domain wall. With reduced pinning, in principle, an unlimited increase of this contribution is possible. In films with the ferroelastic domain pattern, the domain contribution is limited by the clamping effect of the substrate. In the case of not very thin films where the pattern is dense, the upper limit of this contribution can be readily estimated in terms of the approach developed by Roytburd for the description of the 
TABLE IV. Thickness of a dielectric layer with $\varepsilon_{d}=1$, which impacts the dielectric permittivity of the film identically to the effects listed in the first column. $h$-film thickness; $d$-thickness of the layer; $\xi$-correlation radius; $\varepsilon$ and $\varepsilon_{b}$-permittivity and background permittivity of the ferroelectric; $\lambda$-extrapolation length for the polarization boundary conditions; $l_{s}$-Thomas-Fermi screening length; $\beta$-coefficient of the dielectric nonlinearity, $\rho_{0}$-space charge density in the depletion layer; $W$-depletion layer width.

\begin{tabular}{ccc}
\hline \hline & $\begin{array}{c}\text { In-plane } \\
\text { permittivity }\end{array}$ & $\begin{array}{c}\text { Out-of-plane } \\
\text { permittivity }\end{array}$ \\
\hline $\begin{array}{c}\text { Real layer with } \varepsilon_{d}=1 \\
\text { Full surface blocking } \\
\text { of polarization }\end{array}$ & $d$ & $d$ \\
Partial surface blocking & $\xi$ & $\xi / \sqrt{\varepsilon \varepsilon_{b}}$ \\
of polarization & $\frac{\xi}{1+\lambda / \xi}$ & $\frac{\xi / \sqrt{\varepsilon \varepsilon_{b}}}{1+\lambda \sqrt{\varepsilon / \varepsilon_{b}} / \xi}$ \\
$\begin{array}{c}\text { Electrode effect } \\
\text { Partial depletion effect, } h>2 W\end{array}$ & $\ldots$ & $l_{s}$ \\
Full depletion effects, $h<2 W$ & $\ldots$ & $h_{d}=\varepsilon_{0} \beta \rho_{0}^{2} W^{3}$ \\
\hline \hline
\end{tabular}

equilibrium domain pattern in ferroelectric thin films. ${ }^{186,192,193}$ In this case, it can be readily shown that the domain contribution can be of the order of the lattice contribution, having a temperature dependence very similar to the latter. A detailed theory of the domain contribution due to dense $a / c$ and $a_{1} / a_{2}$ pattern in (001) $\mathrm{PbTiO}_{3}$ films has been developed by Koukhar et al. ${ }^{190}$ In thinner films, where the domain spacing is comparable to or larger than the domain thickness, according to Pertzev et al. ${ }^{385}$ the domain contribution due to the $a / c$ pattern may be essentially larger than that of the lattice. An interesting feature of the domain contribution controlled by the substrate clamping is that it is independent of domain wall density (the period of the pattern). This constitutes a striking difference compared to the case of domains in a mechanically free ferroelectric crystal, where the motion of domain walls is controlled by local pinning forces and the domain contribution to the permittivity is expected to be roughly proportional to the domain wall density.

There exist several physical mechanisms which influence the dielectric permittivity of a thin film, such as a dielectric layer with low dielectric permittivity at its surface. These mechanisms are related to the effect of finite thickness of the free-charge layer in the electrode, to the effect of full or partial blocking of the polarization at the surface of the ferroelectric film, and to the depletion effect. A detailed discussion of these mechanisms can be found in Ref. 207. Here we will give a table that enables a comparison of the impact of these mechanisms. In Table IV, a thickness, $d_{\mathrm{eff}}$, of a thin surface dielectric layer (with relative permittivity 1), which produces the same effect on the dielectric permittivity of the film, $\varepsilon_{\text {tot }}$, as the given mechanisms. This means that in terms of $d_{\text {eff }}$ the total (effective) out-of-plane component of the permittivity can be expressed as

$$
\varepsilon_{\mathrm{tot}}^{-1}=\varepsilon^{-1}+\frac{2 d_{\mathrm{eff}}}{h}
$$

where $\varepsilon$ is the permittivity of the ferroelectric and $h$ is the film thickness. The obvious relation for the in-plane component of the permittivity reads
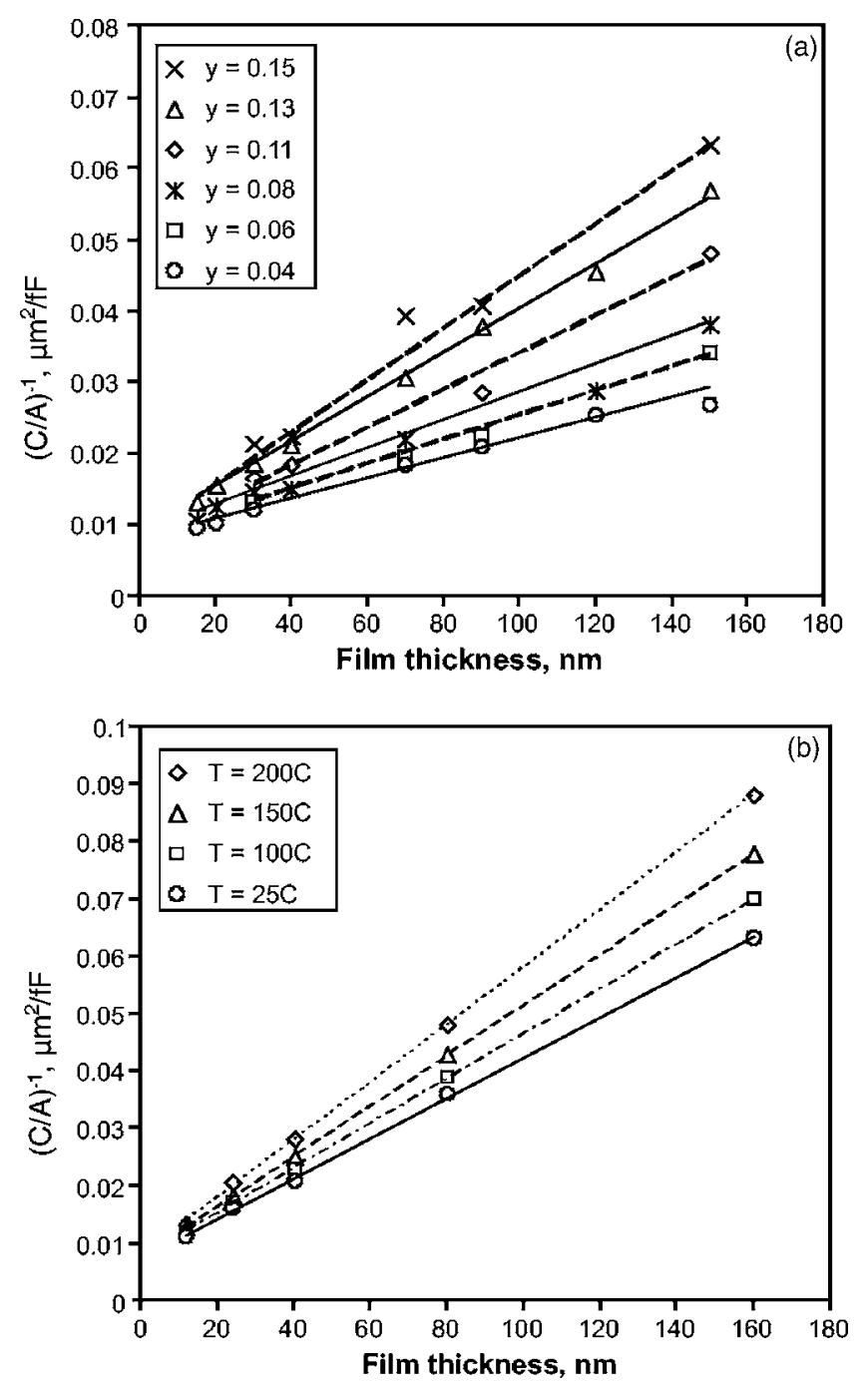

FIG. 40. (a) Film-thickness dependence of the inverse zero-bias capacitance density of $\left(\mathrm{Ba}_{0.7} \mathrm{Sr}_{0.3}\right) \mathrm{Ti}_{1+y} \mathrm{O}_{3+z}$-film-based plate capacitors at different $\mathrm{Ti}$ contents $y$ (Ref. 382); (b) the inverse of the zero-bias capacitance density of $\mathrm{Ba}_{0.7} \mathrm{Sr}_{0.3} \mathrm{TiO}_{3}$ film based plate capacitors as a function of film thickness at different temperatures (Ref. 386).

$$
\varepsilon_{\mathrm{tot}}=\varepsilon \frac{h-2 d_{\mathrm{eff}}}{h}
$$

[Note that, in Eqs. (10) and (11), it is assumed that $h \gg d_{\text {eff. }}$ ]

An analysis of the typical parameters of the ferroelectric and electrode shows that the impact of these mechanisms on the in-plane component of the permittivity is expected to be very small (hardly detectable experimentally), while the impact of all these mechanisms on the out-of-plane component of the permittivity can be appreciable (readily detectable in $300 \mathrm{~nm}$ thick films with parameters of PZT). The estimated values of $d_{\text {eff }}$ are of a fraction of angstrom. ${ }^{207}$ Similar values are obtained experimentally, e.g., in $(\mathrm{Ba}, \mathrm{Sr}) \mathrm{TiO}_{3}$ films. ${ }^{382,386,387}$ Figure 40 illustrates the thickness dependence in $(\mathrm{Ba}, \mathrm{Sr}) \mathrm{TiO}_{3}$ films, which is compatible with Eq. (10).

In terms of equivalent circuits, all the above effects represent the in-series connection of the ferroelectric capacitor with another capacitor of much larger capacitance. This approach is fully justified in the case where only the lattice contribution is essential to the total permittivity of the films. 
If, however, the domain contribution to the permittivity is appreciable, the in-series equivalent-circuit approach may fail, because the stray fields of the domain pattern are not taken into account. The effect of stray fields on the dielectric response has been theoretically treated for the case of a capacitor which contains two thin electrode-adjacent layers and a ferroelectric film with a symmetric $180^{\circ}$ domain pattern, whose domain walls cross the whole thickness of the ferroelectric. $^{200,388,389}$ It has been shown that the equivalentcircuit approach may give a nonadequate description. However, in the case of a dense domain pattern, which is often of practical interest, the system can be still modeled to a good approximation as an in-series connection of a ferroelectric capacitor and a capacitor reproducing the effect of the dielectric layers. Specifically, in this case, the effective permittivity of the system can be found from the relation: ${ }^{389}$

$$
\frac{h+d}{\varepsilon_{\mathrm{eff}}}=\frac{h}{\varepsilon_{c}+\varepsilon_{m}}+\frac{d}{\epsilon_{d}}\left[1-\frac{\varepsilon_{m}^{2}}{\left(\varepsilon_{c}+\varepsilon_{m}\right)^{2}} \xi\right],
$$

where $\varepsilon_{d}$ and $d$ are the permittivity and the total thickness of the dielectric layers, and $\varepsilon_{c}$ and $\varepsilon_{m}$ are the lattice and domain contributions to the permittivity of the ferroelectric. The factor $\xi$ acquires values between 0 and 1 , and is a function of all the parameters of the problem except for the film thickness and the spontaneous polarization of the ferroelectric. In the case of a passive layer much thicker than the domain spacing, $\xi \rightarrow 0$ and Eq. (12) reduces to the standard in-series formula. However, in the opposite case, $\xi \rightarrow 1$ and the impact of the passive layer on the total permittivity is reduced. This reduction can be essential if the permittivity of the ferroelectric is dominated by the domain contribution. This leads to the important conclusion that there may be a situation where the impact of the passive layer on the extrinsic and intrinsic contributions may be very different.

Another effect that can substantially modify the dielectric permittivity of ferroelectric thin films is the poling effect of the electrode/ferroelectric interface. Recent phenomenological analysis performed by Bratkovsky and Levanyuki ${ }^{390}$ has shown that the symmetry breaking introduced by the electrode/ferroelectric interface may effectively pole the ferroelectric, if the electrodes are not identical. According to these authors, in this case the impact of nonidentical electrodes is similar to that of an external dc bias field. This should result in a smearing of the dielectric anomaly and a reduction of the dielectric permittivity. From very rough estimates, this effect may explain a smearing of the dielectric anomaly by some $100 \mathrm{~K}$.

The problem of dielectric loss in thin films is of importance first of all because of their microwave applications. The most common system in this context is $(\mathrm{Ba}, \mathrm{Sr}) \mathrm{TiO}_{3}$ films in the paraelectric phase, where the dielectric losses are acceptable for these applications. The losses in thin films have been usually found higher than in the bulk materials. For $\mathrm{SrTiO}_{3}$ this difference may be of over one order of magnitude. $^{391}$

The frequency dependence of the loss tangent in the $\mathrm{rf}$ range is typically very slow. Figure 41(a) illustrates this point with the data by Baniecki et al. ${ }^{392}$ In the microwave range, the frequency dependence of the loss tangent becomes much
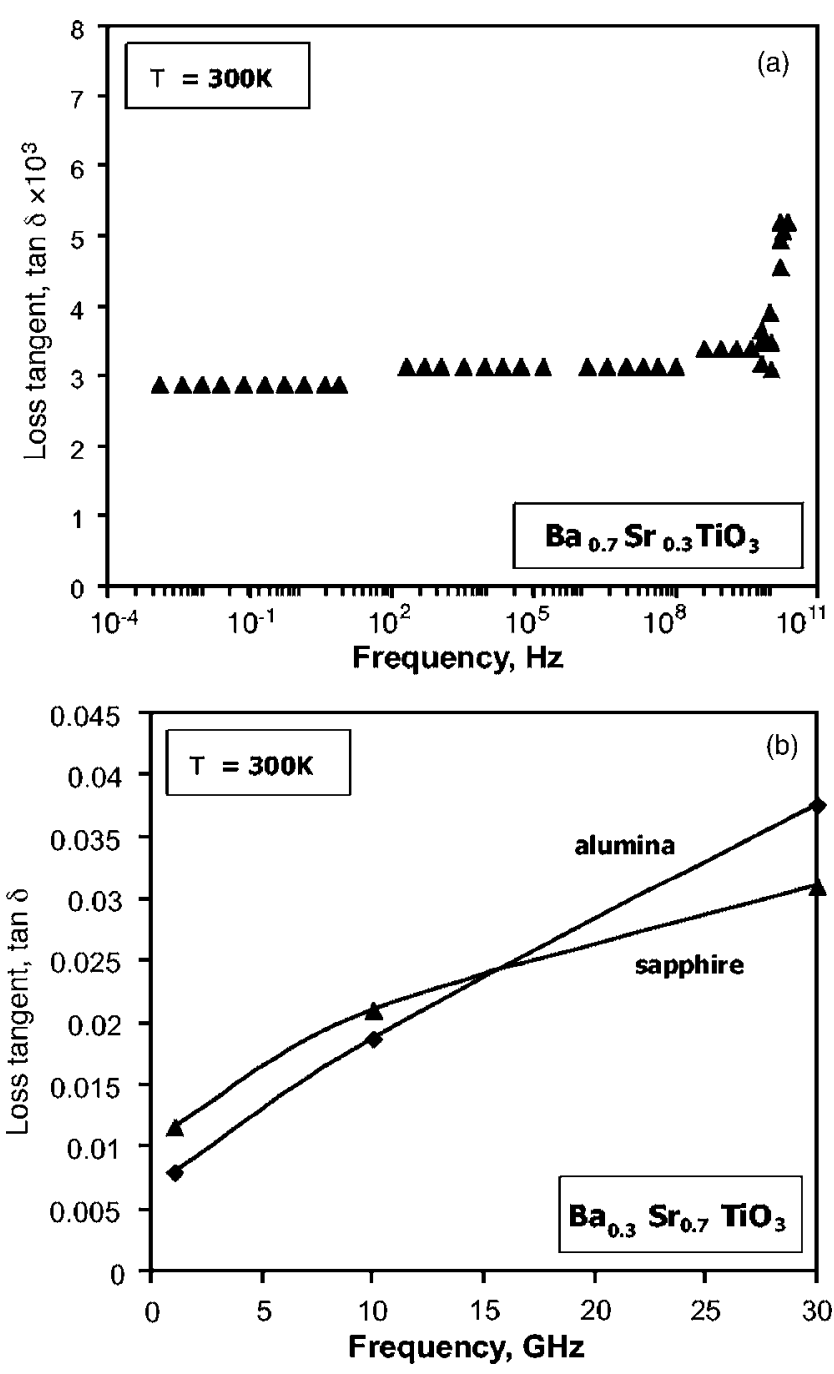

FIG. 41. Frequency dependences of the loss tangents of (a) $\mathrm{Ba}_{0.7} \mathrm{Sr}_{0.3} \mathrm{TiO}_{3}$ thin films (Ref. 392) and (b) $\mathrm{Ba}_{0.3} \mathrm{Sr}_{0.7} \mathrm{TiO}_{3}$ thin films deposited onto alumina and single crystal sapphire substrates (Ref. 393).

faster [often close to linear, Fig. 41(b) cf., e.g., Ref. 393]. The rf data are compatible with the so-called "universalrelaxation-law" mechanism. ${ }^{394}$ In thin films, this mechanism may be associated with field-induced variations of the charge transport barriers. ${ }^{395,396}$ The probable loss mechanisms in the microwave range are those owing to charged defects ${ }^{207,397}$ and local polar regions. ${ }^{398}$ In contrast to single crystal $\mathrm{SrTiO}_{3}$ where the intrinsic (lattice) contribution to the total loss can be appreciable, in thin films the observed values of the loss tangent at microwave frequencies are too high to be attributed to the lattice contribution.

In view of the possible use of ferroelectric thin films in tunable microwave devices, the dc field dependence of the loss tangent becomes an important issue. Typically, the loss tangent is a decreasing function of the dc bias field. ${ }^{399}$ This behavior may be attributed to the combined effect of the increasing dependence of the intrinsic loss mechanisms on the dielectric permittivity and a decreasing dependence of the latter on the dc field. The field dependence of the loss tangent is not always decreasing. An example of increasing and nonmonotonic field dependences of microwave loss have been reported for $\mathrm{SrTiO}_{3}$ films on $\mathrm{MgO}$ substrate. ${ }^{400}$ It has 

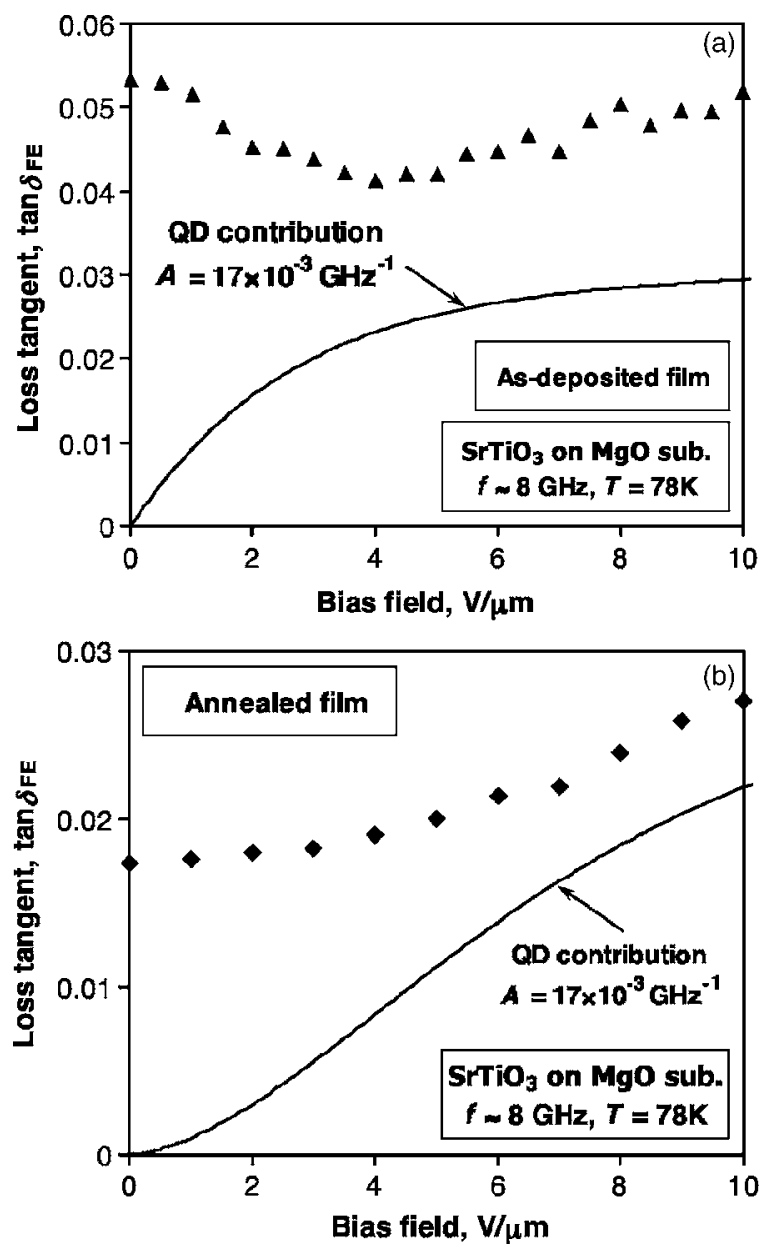

FIG. 42. Experimental field dependences of the loss tangent of as-deposited (a) and annealed (b) $\mathrm{SrTiO}_{3}$ thin films on $\mathrm{MgO}$ substrate (dots) plotted together with the estimates for the contribution of quasi-Debye loss mechanism (lines). (Ref. 400).

been found that in as-deposited films, the loss first decreases and then increases with increasing field, whereas, in annealed films, an increasing dependence has been documented at any fields (Fig. 42). This behavior has been interpreted in terms of the combined action of an extrinsic mechanism with a decreasing field dependence and the field-induced intrinsic quasi-Debye contribution $^{401}$ with an increasing field dependence. In the case of as-deposited films, the decreasing contribution of the extrinsic mechanism dominates the initial reduction of the loss, whereas at higher dc fields, the quasiDebye contribution becomes appreciable, determining the increase of the losses. In the annealed films, the extrinsic contribution is smaller, so that the trend in the loss variation is always controlled by the quasi-Debye contribution. The experimental data are compatible with the results of calculations in terms of the phonon transport theory. ${ }^{402}$

All in all, the losses in ferroelectric thin films are strongly dominated by the extrinsic contribution and, for this reason, are very sensitive to the processing conditions. ${ }^{393,402}$ Though some of their features can be rationalized in terms of theoretical models, the present understanding of the problem is not enough to provide reliable guidelines for tailoring the loss performance in thin film devices.

\section{EMERGING AREAS AND CONCLUSION}

While not yet undergoing a true breakthrough, several additional areas attract interest among ferroelectric researchers and show potential for growth: ferroelectric nanostructure and magnetoelectrics and other multiferroic materials and structures are probably the most visible topics.

\section{A. Ferroelectric 1D nanostructures}

The interest in small anisotropic ferroelectric structures such as disks, rods, wires, and tubes of nanometric dimensions falls within the general interest in such structures following the discovery of carbon nanotubes ${ }^{403}$ and of the possibility to synthesize nanotubes and nanorods of inorganic materials including oxides. ${ }^{404,405}$ Advances have been made both in preparation and testing of such nanomaterials and in modeling of the resulting properties.

Recently $a b$ initio calculations predicted ferroelectric phase transitions in nanodisks of PZT with diameters as low as $3.2 \mathrm{~nm} .{ }^{406}$ It is interesting also to note that $\mathrm{BN}$ which has been synthesized in nanotube forms since some years is predicted to have large electric polarization along the nanotube axis. ${ }^{407,408}$

An attractive way to prepare nanorods is to use nanoporous silicon or alumina as templates and to fill them up with the suitable polymeric precursors which are then heat treated to form the ferroelectric oxide (barium titanate, PZT, etc.) and subsequently separated from the template by etching the latter away. This process was demonstrated on various ferroelectric materials and resulted also in ordered nanorod array. ${ }^{409,410}$

Various chemical processing routes show promise for ferroelectric nanowire fabrication; solution phase decomposition of barium titanium isopropoxide to produce barium titanate [BTO] nanowires with diameters of 5-60 nm and lengths up to $10 \mu \mathrm{m}$ was demonstrated. ${ }^{411}$ A molten salt technique was used to produce BTO nanowires in a large scale fashion. ${ }^{412}$ BTO and strontium titanete nanotubes were produced by reacting titania nanotubes with barium and strontium hydroxides. ${ }^{413}$ Hydrothermal growth of potassium niobate nanowires was demonstrated. ${ }^{44}$ The hydrothermal method was used also for the fabrication of arrays of BTO nanotubes on titanium substrate. ${ }^{415}$

Very little has been reported so far on the mechanism by which nanotubes and nano wires grow. ${ }^{416}$ Likewise, there are very few detailed reports on the ferroelectric properties of nanotubes and nanowires. However, there are two recent reports where switching of the ferroelectric polarization and local piezoelectric activity have been detected using force microscopy. ${ }^{417,418}$ These hold a promise that in the foreseeable future we shall see rapid progress in this direction.

\section{B. Multiferroics}

The so-called "multiferroic" materials are those that display more than one ferroic property (ferroelectric, ferromagnetic, and ferroelastic). (The term multiferroic has been ascribed to $\mathrm{Schmid}^{419}$ ). The term most properly refers to the coexistence of these properties in a single phase material, 
although the term has also been more loosely applied to composite materials in which the ferroic behavior exists individually within separate phases.

While it has been known for a considerable time ${ }^{419-422}$ that these properties can coexist and that the properties may be coupled, there has been a recent renewed interest in the topic. This has been fueled by reports of large remanent polarization and weak saturation magnetization values in thin films of $\mathrm{BiFeO}_{3} \cdot{ }^{423}$ Interestingly, both the magnetization and polarization values were shown to be strongly dependent on film thickness, increasing as film thickness was reduced. The initial report therefore suggested that the properties correlated with epitaxial strain of the films. However, this is not yet clear, with oxygen vacancy concentration also being suggested as a primary contributory factor, and more recent reports (both experimental and theoretical) suggesting that oxygen vacancies and strain should not have a significant direct impact upon remanent polarization. ${ }^{424-428}$ The straincontrolled idea was particularly interesting, as it was followed by a paper showing that epitaxial strain could assert a remarkable influence on the transition temperature of $\mathrm{SrTiO}_{3},{ }^{208}$ adding weight to an argument for further studies of strain-engineered ferroic properties. More recently, it has been suggested that it is not the high polarization values in $\mathrm{BiFeO}_{3}$ that are anomalous, but rather the values of Ps in $\mathrm{BiFeO}_{3}$ single crystals are anomalously low. For example, recent work has suggested that the polarization is physically constrained within crystals of normal geometry. As it is, the mechanisms operative in $\mathrm{BiFeO}_{3}$ thin films (and single crystals) clearly require further investigation. Nonetheless, the possibility of a high polarization, lead-free ferroelectric thin film material is of significant interest for the community.

There are a number of materials that possess multiple ferroic properties, but the reports of coupling between these properties are significantly more limited. In the case of magnetoelectric materials, the manifestation of coupling between magnetic and electric parameters has primarily been the small modulation of the spontaneous polarization or dielectric properties by a magnetic field, or the presence of anomalies at phase transitions. However, there have been extremely few reports of the complete control or reversal of ferroelectric polarization by an applied magnetic field ${ }^{420,429-431}$ and none of them has been achieved at room temperature.

This points to the need for a search for thin film multiferroic materials. However, Hill has postulated some fundamental reasons that there are so few magnetic ferroelectrics. ${ }^{432}$ She has pointed out that the $d$ electrons necessary for magnetism reduce the tendency for the structural distortion essential for ferroelectricity. Similarly, she observe that the presence of the Jahn-Teller distortion present in many ferroelectrics appears to inhibit the appearance of magnetoferroelectrics. ${ }^{432}$

The potential dearth of interesting multiferroic structures that may be grown under equilibrium conditions underlines the potential importance of artificial superlattice structures. This field remains in its infancy, with increasing capability in the community for synthesis of true complex oxide superlattice structures that display properties. ${ }^{240,433-436}$
It is important to note that the preceding discussion refers to single phase multiferroic materials. It is of course possible to obtain coupling between two different ferroic materials, constructed as a multiphase composite. For example, macroscopic multiferroic multilayers based on piezoelectric/ magnetostrictive coupling have been demonstrated. ${ }^{437}$ However, it is essential to appropriately engineer the composite microstructures in order to maximize responses and coupling. Thus the piezoelectric/ferroelectric composite materials grown as rods of ferromagnetic material perpendicular to the substrate within a matrix of ferroelectric material would be expected to show improved coupling due to minimal substrate constraint. $^{438}$ Furthermore, an elegant method of growth to achieve these microstructures that relies on simultaneous deposition of two epitaxial phases has been demonstrated for the $\mathrm{CoFe}_{2} \mathrm{O}_{4}-\mathrm{BaTiO}_{3},{ }^{438} \mathrm{CoFe}_{2} \mathrm{O}_{4}-\mathrm{PbTiO}_{3}{ }^{439}$ and $\mathrm{CoFeO}_{3}-\mathrm{BiFeO}_{3}$ (Ref. 440) systems. In the case of the last system, there is direct evidence of switching of the magnetization by application of an electric field to the composite. This may be evidence of room temperature multiferroic switching, albeit in a multiphase system. Also interesting, although without direct evidence of switching, is the report of field-induced modulation of the magnetic moment in the dilute magnetic insulator, Co-doped $\mathrm{TiO}_{2}{ }^{441}$

An important question is whether these multiferroic thin film materials have real potential for memory applications. The concomitant investigation of memory devices based upon multiferroic films is strongly lacking. There needs to be concerted investigations to determine whether there may be memory or other devices in which multiferroic coupling provides unique and exploitable advantages.

\section{Conclusion}

We attempted in this paper to describe the state of art in the field of ferroelectric thin films. Applications were the starting point and we reviewed three main areas of applications: microsystems containing ferroelectrics, high frequency components with polar films, and the field of ferroelectric memories. Based on these applications we discussed materials, domains in ferroelectric thin films, and size effects. Several important properties that are essential for the applications of ferroelectrics were presented in an extended way and finally we remarked on the emerging areas of magnetoelectrics and nanoscale 1D ferroelectric structures.

Research in field of ferroelectric films continues to be application driven. While numerous interesting questions are still open and, no doubt, a number of materials and structures are there yet to be elaborated, further developments depend on the "market pull," namely, on the speed at which the related applications will expand in the near future.

\section{ACKNOWLEDGMENTS}

This review is part of the activity of MIND-European Network on Piezoelectrics (6th FP). The European Commission is acknowledged for the financial support [to one of the authors (N.S.)]. Acknowledgments are given also to the Swiss National Science Foundation [to four of the authors (I.S., T.Y., A.K.T., and N.S.)], to the Volkswagen-Stiftung 
Project "Nano-sized Ferroelectric Hybrids" under Contract No. I/77737 and that of the Deutsche Forschungsgemeinschaft (DFG) [to one of the authors (H.K.)], and to the Deutsche Forschungsgemeinschaft DFG under Project No. FOR 520 [to another author (L.E.)]. Matthew Davis and Guido Gerra are acknowledged for correcting the manuscript.

${ }^{1}$ D. W. Chapman, J. Appl. Phys. 40, 2381 (1969).

${ }^{2}$ M. H. Francombe, Thin Solid Films 13, 243 (1972).

${ }^{3}$ B. S. Sharma, S. F. Vogel, and P. I. Prentky, Ferroelectrics 5, 69 (1973).

${ }^{4}$ J. B. Blum and S. R. Gurkowich, J. Mater. Sci. 20, 4479 (1985).

${ }^{5}$ K. D. Budd, S. K. Dey, and D. A. Payne, Br. Ceram. Proc. 36, 107 (1988).

${ }^{6}$ S. S. Eaton, D. B. Butler, M. Parris, D. Wilson, and H. McNeillie, Dig. Tech. Pap.-IEEE Int. Solid-State Circuits Conf. 130, 329 (1988).

${ }^{7}$ C. P. de Araujo, G. W. Taylor, and J. F. Scott, in Ferroelectric Thin Films: Synthesis and Basic Properties, edited by C Paz de Araujo, G. W. Taylor, and J. F. Scott (Gordon and Breach, Amsterdam, 1996).

${ }^{8}$ R. Ramesh, in Thin Film Ferroelectric Materials and Devices, edited by R. Ramesh (Kluwer Academinc, Dordrecht, 1997).

${ }^{9}$ N. Setter, Electroceramic Based MEMS (Springer, New York, 2005).

${ }^{10}$ J. F. Scott and C. A. P. de Araujo, Science 246, 1400 (1989).

${ }^{11}$ M. Dawber, K. M. Rabe, and J. F. Scott, Rev. Mod. Phys. 77, 1083 (2005).

${ }^{12}$ A. Kingon, P. Muralt, N. Setter, and R. Waser, Ceramic Materials for Electronics, edited by R. E. Buchanan (Dekker, New York, 2004), pp. 465-526.

${ }^{13}$ P. Muralt, J. Micromech. Microeng. 10, 136 (2000).

${ }^{14}$ www.ir-microsystems.com

${ }^{15}$ T. Maeder, P. Muralt, M. Kohli, A. Kholkin, and N. Setter, Br. Ceram. Proc. 54, 206 (1995).

${ }^{16}$ P. Muralt, J. Baborowski, and N. Ledermann, in Piezoelectric Materials in Devices, edited by N. Setter (Ceramics Laboratory, EPFL, Lausanne, Switzerland, 2002), Chap. 12, pp. 231-260.

${ }^{17}$ A. M. Flynn, L. S. Tavrow, S. F. Bart, R. A. Brooks, D. J. Ehrlich, K. R Udayakumar, and L. E. Cross, J. Microelectromech. Syst. 1, 44 (1992).

${ }^{18}$ M. A. Dubois and P. Muralt, IEEE Trans. Ultrason. Ferroelectr. Freq Control 45, 1169 (1998).

${ }^{19}$ P. Luginbuhl, S. D. Collins, G. A. Racine, M. A. Grétillat, N. F. de Rooij, K. G. Brooks, and N. Setter, J. Microelectromech. Syst. 6, 337 (1997).

${ }^{20}$ Y. Nemirovsky, A. Nemirovsky, P. Muralt, and N. Setter, Sens. Actuators, A 56, 239 (1996).

${ }^{21}$ N. Ledermann, J. Baborowski, A. Seifert, B. Willing, S. Hiboux, P. Muralt, N. Setter, and M. Forster, Integr. Ferroelectr. 35, 177 (2001).

${ }^{22}$ T. Fujii and S. Watanabe, Appl. Phys. Lett. 68, 467 (1996).

${ }^{23}$ J. Y. Park, Y. J. Yee, H. J. Nam, and J. U. Bu, IEEE MTT-S Int. Microwave Symp. Dig. 2001, 2111.

${ }^{24}$ J. Baborowski, N. Ledermann, P. Muralt, and D. Schmitt, Int. J. Comput. Eng. Sci. 4, 471 (2003).

${ }^{25}$ J. J. Bernstein et al., IEEE Trans. Ultrason. Ferroelectr. Freq. Control 44, 960 (1997).

${ }^{26}$ T. Yoshimura and S. Trolier-McKinstry, J. Appl. Phys. 92, 3979 (2002).

${ }^{27}$ Z. Kighelman, D. Damjanovic, and N. Setter, J. Appl. Phys. 90, 4682 (2001).

${ }^{28}$ S. Bühlmann, B. Dwir, J. Baborowski, and P. Muralt, Appl. Phys. Lett. 80, 3195 (2002).

${ }^{29}$ N. Ledermann, P. Muralt, J. Baborowski, S. Gentil, K. Mukati, M. Cantoni, A. Seifert, and N. Setter, Sens. Actuators, A 105, 162 (2003).

${ }^{30}$ J. Quyang, R. Ramesh, and A. L. Roytburd, Appl. Phys. Lett. 86, 152901 (2005).

${ }^{31}$ R. Maeda, T. T. Tsaur, S. H. Lee, and M. Ichicki, in Electroceramic Based $M E M S$, editer by N. Setter (Springer, New York, 2005), Chap. 2, pp. $19-36$.

${ }^{32}$ S. J. Gross, S. Tadigadapa, T. N. Jackson, S. Trolier-McKinstry, and Q. Q. Zhang, Appl. Phys. Lett. 83, 1 (2003).

${ }^{33}$ Y. S. Kim, C. S. Lee, W. H. Jin, S. S. Jang, H. J. Nam, and J. U. Bu, Sens. Mater. 17, 57 (2005).

${ }^{34}$ P. V. M. Despont et al., IBM J. Res. Dev. 44, 323 (2000).

${ }^{35}$ P. Muralt, in Electroceramic Based MEMS, edited by N. Setter (Springer, New York, 2005), Chap. 3, pp. 37-48.

${ }^{36} \mathrm{~J}$. Baborowski, Electroceramic Based MEMS, edited by N. Setter (Springer, New York, 2005), Chap. 13, pp. 325-360.

${ }^{37}$ G. Percin and B. T. Khuri-Yakub, Rev. Sci. Instrum. 73, 2193 (2002).

${ }^{38}$ K. Yamashita et al., Sens. Actuators, A 97, 302 (2002).
${ }^{39}$ K. M. Lakin and J. S. Wang, Appl. Phys. Lett. 38, 125 (1981).

${ }^{40}$ K. M. Lakin, G. R. Kline, and K. T. McCarron, IEEE Trans. Microwave Theory Tech. 43, 2933 (1995).

${ }^{41}$ P. Muralt and R. Lanz, in Piezoelectric Materials in Devices, edited by N. Setter (Ceramics Laboratory, EPFL, Lausanne, Switzerland, 2002), Chap. 15, pp. 303-314.

${ }^{42}$ N. Ylilammi, J. Ella, M. Partanen, and J. Kaitila, IEEE Trans. Ultrason. Ferroelectr. Freq. Control 49, 535 (2002).

${ }^{43}$ F. Martin, P. Muralt, and M. A. Dubois, J. Vac. Sci. Technol. A 22, 361 (2004).

${ }^{44}$ A. Ballato, IEEE Trans. Ultrason. Ferroelectr. Freq. Control 41, 834 (1994).

${ }^{45}$ M. A. Dubois and P. Muralt, Appl. Phys. Lett. 74, 3032 (1999).

${ }^{46}$ J. Kaitila, M. Yililammi, J. Molaris, J. Ellä, and T. Makkonen, Proc.-IEEE Ultrason. Symp. 803 (2001).

${ }^{47}$ P. Muralt and R. Lanz, in Piezoelectric Materials in Devices, edited by N. Setter (Ceramics Laboratory, EPFL, Lausanne, Switzerland, 2002), Chap. 15 , p. 312.

${ }^{48}$ www.semiconductors.philips.com and www.infineon.com

${ }^{49}$ H. P. Loebl, C. Metzmacher, R. F. Milsom, P. Lok, and A. Tuinhout, in Electroceramic Based MEMS, edited by N. Setter (Springer, New York, 2005), Chap. 6, p. 115.

${ }^{50} \mathrm{~S}$. Gevorgian, in Electroceramic-Based MEMS, edited by N. Setter (Springer, New York, 2005), Chap. 7, p. 133.

${ }^{51}$ A. Vorobiev, P. Rundqvist, K. Khamchane, and S. Gevorgian, Appl. Phys. Lett. 83, 3144 (2003).

${ }^{52}$ V. E. Demidov, B. A. Kalinikos, S. F. Karmanenko, A. A. Semenov, and P. Edenhofer, IEEE Trans. Microwave Theory Tech. 51, 2090 (2003).

${ }^{53}$ Q. X. Jia, J. R. Groves, P. Arendt, Y. Fan, A. T. Findikoglu, S. R. Foltyn, H. Jiang, and F. A. Miranda, Appl. Phys. Lett. 74, 1564 (1999).

${ }^{54}$ S. Gevorgian, S. Abadei, H. Berg, and H. Jacobsson, IEEE MTT-S Int. Microwave Symp. Dig. 2, 1195 (2001).

${ }^{55}$ H. H. Fiallo, J. P. Dougherty, S. J. Jang, R. E. Newnham, and L. A. Carpenter, IEEE Trans. Microwave Theory Tech. 42, 1176 (1994).

${ }^{56}$ S. Gevorgian and E. Kollberg, IEEE Trans. Microwave Theory Tech. 49, 2117 (2001).

${ }^{57}$ S. Gevorgian, IEEE International Microwave Symposium Workshop on Ferroeletric Materials and Devices for Microwave Applications, Philadelphia, 2003 (unpublished).

${ }^{58}$ V. V. Lemanov, A. V. Sotnikov, E. P. Smirnova, M. Weihnacht, and R. Kunze, Solid State Commun. 110, 611 (1999).

${ }^{59}$ E. Kollberg, J. Stake, and L. Dillner, Philos. Trans. R. Soc. London, Ser. A 354, 2383 (1996).

${ }^{60} \mathrm{http}: / /$ agilematerials.com

${ }^{61} \mathrm{http}: / /$ www.globalspec.com/ParatekMicrowave

${ }^{62}$ S. Gevorgian, P. K. Petrov, Z. Ivanov, and E. Wikborg, Appl. Phys. Lett. 79, 1861 (2001).

${ }^{63}$ S. Gevorgian, H. Jacobsson, and T. Lewin, U.S. Patent Application No. PCT/SE 2004/00329 (2004).

${ }^{64}$ O. G. Vendik and S. P. Zubko, J. Appl. Phys. 82, 4475 (1997).

${ }^{65}$ Ferroelectric Random Access Memories, of Topics in Applied Physics, vol. 93, edited by H. Ishiwara and M. Okuyama (Springer-Verlag, Berlin, 2004).

${ }^{66}$ J. F. Scott, Ferroelectric Memories, Springer Series in Advanced Microelectronics (Springer-Verlag, Berlin, 2000).

${ }^{67}$ J. Evans and R. Womack, IEEE J. Solid-State Circuits 23, 1171 (1988).

${ }^{68} \mathrm{R}$. Womack and D. Tolsch, Dig. Tech. Pap.-IEEE Int. Solid-State Circuits Conf. 1989 , p. 242

${ }^{69}$ C. A. P. de Araujo, J. D. Cuchiaro, L. D. MacMillan, M. C. Scott, and J. F. Scott, Nature (London) 374, 627 (1995).

${ }^{70}$ T. Sumi et al., Dig. Tech. Pap.-IEEE Int. Solid-State Circuits Conf. 1994, p. 268.

${ }^{71}$ E. Choi et al., Integr. Ferroelectr. 66, 107 (2004).

${ }^{72}$ S. Sinharoy, H. Buhay, D. R. Lampe, and M. H. Francombe, J. Vac. Sci. Technol. A 10, 1554 (1992).

${ }^{73}$ H. Volz, K. Koger, and H. Schmitt, Ferroelectrics 56, 161 (1984).

${ }^{74}$ T. Hayashi et al., Tech. Dig. - Int. Electron Devices Meet. 2002.

${ }^{75}$ H. Kim, S. Yamamoto, and H. Ishiwara, Integr. Ferroelectr. 67, 271 (2004).

${ }^{76}$ F. Chu, G. Fox, and T. Davenport, Integr. Ferroelectr. 36, 43 (2001).

${ }^{77}$ F. Chu, G. Fox, T. Davenport, Y. Miyaguchi, and K. Suu, Integr. Ferroelectr. 48, 161 (2002).

${ }^{78}$ A. Itoh et al., Proc. IEEE VLSI Tech. Symposium, 2000.

${ }^{79}$ W. Kraus, L. Lehman, D. Wilson, T. Yamasaki, C. Ohno, E. Nagai, H. 
Yamazaki, and H. Suziki, Symp. VLSI Circuits, 1998, p. 242.

${ }^{80}$ G. R. Fox and S. Summerfelt, in Magnetic and Electronic Films -Microstructure, Texture and Application to Data Storage, edited by P. W. DeHaven, D. P. Field, S. D. Harkness, J. A. Sutliff, J. A. Szpunar, L. Tang, T. Thomson, and V. D. Vaudin (Materials Research Society, Pittsburgh, PA, 2002), p. 145.

${ }^{81}$ E. Fujii and K. Uchiyama, Integr. Ferroelectr. 53, 317 (2003).

${ }^{82}$ Y. Horii et al., Tech. Dig. - Int. Electron Devices Meet. 2002, p. 539

${ }^{83}$ H. J. Joo et al., Integr. Ferroelectr. 68, 139 (2004).

${ }^{84}$ T. S. Moise et al., Tech. Dig. - Int. Electron Devices Meet. 1999, p. 940

${ }^{85}$ D. Takashima and Y. Oowaki, Ferroelectric Random Access Memories, Topics in Applied Physics Vol. 93, (Springer-Verlag, Berlin, 2004), p. 198. ${ }^{86} \mathrm{H}$. McAdams et al., IEEE J. Solid-State Circuits 39, 1 (2004).

${ }^{87}$ T. S. Moise et al., Tech. Dig. - Int. Electron Devices Meet. 2002, p. 535.

${ }^{88}$ J. A. Rodriguez et al., IEEE Trans. Device Mater. Reliab. 4, 436 (2004)

${ }^{89}$ Y. Arimoto and H. Ishiwara, MRS Bull. 29, 823 (2004).

${ }^{90}$ H. Ishiwara, Mater. Res. Soc. Symp. Proc. 596, 427 (2000).

${ }^{91}$ H. Kohlstedt and H. Ishiwara, in Nanoelectronics and Information Technology: Advanced Electronic Materrial and Novel Devices, edited by R. Waser (Wiley-VCH, Weinheim, 2002), Chap. 14, p. 387.

${ }^{92}$ I. M. Ross, U.S. Patent No. 2,791,760 (1957).

${ }^{93}$ J. L. Moll and Y. Tarui, IEEE Trans. Electron Devices ED-10, 338 (1963)

${ }^{94}$ S. Y. Wu, IEEE Trans. Electron Devices ED-21, 499 (1974).

${ }^{95}$ K. Takahashi, K. Manabe, A. Morioka, T. Ikarashi, T. Yoshihara, H. Watanabe, and T. Tatsumi, Abstracts of the International Conference Solid State Devices and Materials, Tokyo, 2004 (unpublished), Paper No. 1-2.

${ }^{96}$ S. A. Chambers, Y. Liang, Z. Yu, R. Droopad, J. Ramdani, and K. Eisenbeiser, Appl. Phys. Lett. 77, 1662 (2000).

${ }^{97}$ C. J. Förster, C. R. Ashman, K. Schwarz, and P. E. Blöchl, Nature (London) 427, 53 (2004).

${ }^{98}$ R. A. McKee, F. J. Walker, and M. F. Chisholm, Phys. Rev. Lett. 81, 3014 (1998).

${ }^{99}$ I. P. Batra, P. Würfel, and B. D. Silverman, Phys. Rev. Lett. 30, 384 (1973).

${ }^{100}$ R. R. Metha, B. D. Siverman, and J. T. Jacobs, J. Appl. Phys. 44, 3379 (1973).

${ }^{101}$ T. Furukawa, Phase Transitions 18, 143 (1989).

${ }^{102}$ S. H. Lim, A. C. Rastogi, and S. B. Desu, J. Appl. Phys. 96, 5673 (2004).

${ }^{103}$ T. J. Reece, A. Ducharme, A. V. Sorokin, and M. Poulsen, Appl. Phys. Lett. 82, 142 (2003).

${ }^{104}$ N. Yamauchi, Jpn. J. Appl. Phys., Part 1 25, 590 (1986)

${ }^{105}$ R. C. G. Naber, C. Tanase, P. W. M. Blom, G. H. Gelinck, A. W. Marsman, F. J. Touwslager, S. Setayesh, and D. M. De Leeuw, Nat. Mater. 4, 243 (2005).

${ }^{106}$ R. Schroeder, L. Majewski, and M. Grell, Adv. Mater. (Weinheim, Ger.) 16, 633 (2004)

${ }^{107}$ G. H. Gelinck, A. W. Marsman, F. J. Touwslager, S. Setayesh, D. M. de Leeuw, R. C. G. Naber, and P. W. M. Blom, Appl. Phys. Lett. 87, 092903 (2005).

${ }^{108}$ P. W. M. Blom, R. M. Wolf, J. F. M. Cillessen, and M. P. C. M. Krijn, Phys. Rev. Lett. 73, 2107 (1994).

${ }^{109}$ K. Gotoh, H. Tamaura, H. Takauchi, and A. Yoshida, Jpn. J. Appl. Phys., Part 1 35, 39 (1996).

${ }^{110}$ M. Okano and Y. Watanabe, Appl. Phys. Lett. 76, 233 (2000).

${ }^{111}$ R. Meyer and H. Kohlstedt, IEEE Trans. Ultrason. Ferroelectr. Freq. Control (in press).

${ }^{112}$ W. Zhao and D. Jena, Appl. Phys. Lett. 96, 2095 (2004).

${ }^{113}$ L. Esaki, R. B. Laibowitz, and P. J. Stiles, IBM Tech. Discl. Bull. 13, 2161 (1971)

${ }^{114}$ H. Kohlstedt, N. A. Pertsev, and R. Waser, Mater. Res. Soc. Symp. Proc. 688, 161 (2002).

${ }^{115}$ H. Qu, W. Yao, T. Garcia, J. Zhang, A. V. Sorokin, S. Ducharme, P. A. Dowben, and V. M. Fridkin, Appl. Phys. Lett. 82, 4322 (2003).

${ }^{116}$ J. R. Contreras, H. Kohlstedt, U. Poppe, R. Waser, C. Buchal, and N. A. Pertsev, Appl. Phys. Lett. 83, 4595 (2003).

${ }^{117}$ R. Wolf, P. W. M. Blom, and M. P. C. Krijn, U.S. Patent No. 5,541,422 (1996).

${ }^{118}$ M. Indlekofer and H. Kohlstedt, Europhys. Lett. 72, 282 (2005).

${ }^{119}$ M. Y. Zhuravlev, R. F. Sabirianov, S. S. Jaswal, and E. Y. Tsymbal, Phys Rev. Lett. 94, 246802 (2005).

${ }^{120}$ H. Kohlstedt, N. A. Pertsev, J. R. Contreras, and R. Waser, Phys. Rev. B 72, 125341 (2005).

${ }^{121}$ D. P. Oxley, Electrocomponent Sci. Technol. 3, 217 (1977).

${ }^{122}$ D. M. Schaadt, E. T. Yu, V. Vaithyanathan, and D. Schlom, J. Vac. Sci.
Technol. B 22, 2030 (2004).

${ }^{123}$ A. Dietzel, in Nanoelectronics and Information Technology: Advanced Electronic Materials and Novel Devices, edited by R. Waser (WileyVCH, Weinheim, 2002), Chap. 24, p. 617.

${ }^{124}$ G. Binasch, P. Grünberg, F. Saurenbach, and W. Zinn, Phys. Rev. B 39, 4828 (1989).

${ }^{125}$ Nanoscale Characterization of Ferroelectric Materials, Nanoscience and Technology, edited by M. Alexe and A. Gruverman (Springer-Verlag, Berlin, 2004).

${ }^{126}$ Nanoscale Phenomena in Ferroelectric Thin Films, edited by S. Hong (Kluwer Academic, Dordrecht, 2004).

${ }^{127}$ K. Franke, J. Besold, W. Haessler, and C. Seegebrath, Surf. Sci. 302, L283 (1994).

${ }^{128}$ T. Tybell, P. Raruch, T. Giamarchi, and J. M. Trescone, Phys. Rev. Lett. 89, 097601 (2002).

${ }^{129}$ T. Tybell, C. H. Ahn, and J. M. Triscone, Appl. Phys. Lett. 75, 856 (1999).

${ }^{130}$ H. Shin, Nanoscale Phenomena in Ferroelectric Thin Films (Ref. 126), Chap. XI, p. 263.

${ }^{131}$ H. Birk, J. Glatz-Reichenbach, E. Schreck, and K. Dransfeld, J. Vac. Sci. Technol. B 6, 1162 (1991).

${ }^{132}$ Y. Cho, K. Fujimoto, Y. Hiranaga, Y. Wagatsuma, A. Onoe, K. Terabe, and K. Kitamura, Nanotechnology 14, 637 (2003).

${ }^{133}$ T. P. Ma and J.-P. Han, IEEE Electron Device Lett. 23, 386 (2002).

${ }^{134}$ S. R. Summerfelt, in Thin Film Ferroelectric Materials and Devices, edited by R. Ramesh (Kluwer Academic, Norwell, MA, 1997).

${ }^{135}$ H. N. Al-Shareef and A. I. Kingon, in Ferroelectric Thin Films: Synthesis and Basic Properties, edited by C. A. Paz de Araujo, J. F. Scott, and G. W. Taylor (Gordon and Breach, New York, 1996), Chap. 7.

${ }^{136}$ A. K. Tagantsev, I. Stolichnov, E. Colla, and N. Setter, J. Appl. Phys. 90, 1387 (2001)

${ }^{137}$ H. N. Al-Shareef, D. Dimos, W. L. Warren, and B. A. Tuttle, J. Appl. Phys. 80, 4573 (1996).

${ }^{138}$ G. Arlt and H. Neumann, Ferroelectrics 87, 109 (1988).

${ }^{139}$ M. Grossmann, O. Lohse, D. Bolten, U. Boettger, R. Waser, W. Hartner, M. Kastner, and G. Schindler, Appl. Phys. Lett. 76, 363 (2000).

${ }^{140}$ A. Gruverman, B. J. Rodriguez, A. I. Kingon, R. J. Nemanich, J. S. Cross, and M. Tsukada, Appl. Phys. Lett. 82, 3071 (2003).

${ }^{141}$ B. H. Park, B. S. Kang, S. D. Bu, T. W. Noh, J. Lee, and W. Jo, Nature (London) 401, 682 (1999).

${ }^{142}$ A. Lin, X. Hong, V. Wood, A. A. Verevkin, C. H. Ahn, R. A. McKee, F. J. Walker, and E. D. Specht, Appl. Phys. Lett. 78, 2034 (2001).

${ }^{143}$ A. Gruverman, Appl. Phys. Lett. 75, 1452 (1999).

${ }^{144}$ V. Nagarajan, S. Aggarwal, A. Gruverman, R. Ramesh, and R. Waser, Appl. Phys. Lett. 86, 262910 (2005).

${ }^{145}$ H. Funakuboet al., Mater. Sci. Eng., B 118, 23 (2005), and references therein.

${ }^{146}$ T. Oikawa, M. Aratani, K. Saito, and H. Funakubo, J. Cryst. Growth 237-239, 455 (2002).

${ }^{147}$ C. M. Foster, G. R. Bai, R. Csencsits, J. Vetrone, R. Jammy, L. A. Wills, E. Carr, and J. Amano, J. Appl. Phys. 81, 2349 (1997).

${ }^{148}$ B. Jaffe, W. Jaffe, and R. Cook, Piezoelectric Ceramics (Academic, London, 1972).

${ }^{149}$ C. Dehoff, B. J. Rodriguez, A. I. Kingon, R. J. Nemanich, A. Gruverman, and J. S. Cross, Rev. Sci. Instrum. 76, 023708 (2005).

${ }^{150}$ J. Li, H. Liang, B. Nagaraj, W. Cao, C. H. Lee, and R. Ramesh, J. Lightwave Technol. 21, 3283 (2003).

${ }^{151}$ J. Li, B. Nagaraj, H. Liang, W. Cao, C. H. Lee, and R. Ramesh, Appl. Phys. Lett. 84, 1174 (2004).

${ }^{152}$ E. Sviridov, V. Alyoshin, V. Mukhortov, Y. Golovko, V. Dudkevich, and E. Fesenko, Ferroelectrics 56, 1153 (1984).

${ }^{153}$ E. Sviridov, V. Alyoshin, Y. Golovko, I. Zakharchenko, V. Mukhortov, and V. Dudkevich, Ferroelectrics 128, 1 (1992).

${ }^{154}$ H. Odagawa and Y. Cho, Ferroelectrics 251, 29 (2001).

${ }^{155}$ C. S. Ganpule et al., Appl. Phys. Lett. 77, 292 (2000).

${ }^{156}$ A. Seifert, F. F. Lange, and J. S. Speck, J. Mater. Res. 10, 680 (1995).

${ }^{157}$ T. Maruyama, M. Saitoh, T. H. I. Sakai, Y. Yano, and T. Noguchi, Appl. Phys. Lett. 73, 3524 (1998).

${ }^{158}$ A. L. Roytburd, S. P. Alpay, L. A. Bendersky, V. Nagarajan, and R. Ramesh, J. Appl. Phys. 89, 553 (2001).

${ }^{159}$ C. M. Fosteret al., J. Appl. Phys. 78, 2607 (1995).

${ }^{160}$ K. S. Lee and S. Baik, J. Appl. Phys. 87, 8035 (2000).

${ }^{161}$ B. S. Kwak, A. Erbil, B. J. Wilkens, J. D. Budai, M. F. Chisholm, and L. A. Boatner, Phys. Rev. Lett. 68, 3733 (1992). 
${ }^{162}$ R. Ahluwalia and W. Cao, J. Appl. Phys. 89, 8105 (2001).

${ }^{163}$ W. Y. Hsu and R. Raj, Appl. Phys. Lett. 67, 792 (1995).

${ }^{164}$ S. P. Alpay, A. S. Prakash, S. Aggarwal, R. Ramesh, and A. L. Roytburd, Mater. Res. Soc. Symp. Proc. 493, 111 (1998).

${ }^{165}$ Y. M. Kang and S. Baik, J. Appl. Phys. 82, 2532 (1997).

${ }^{166}$ B. S. Kwak, A. Erbil, J. D. Budai, M. F. Chisholm, L. A. Boatner, and B J. Wilkens, Phys. Rev. B 49, 14865 (1994).

${ }^{167}$ K. S. Lee and S. Baik, J. Appl. Phys. 85, 1995 (1999).

${ }^{168}$ K. S. Lee, Y. M. Kang, and S. Baik, Integr. Ferroelectr. 14, 43 (1997).

${ }^{169}$ T. Ogawa, A. Senda, and T. Kasanami, Jpn. J. Appl. Phys., Part 130 , 2145 (1991).

${ }^{170}$ V. Srikant, E. J. Tarse, D. R. Clarke, and J. S. Speck, J. Appl. Phys. 77, 1517 (1995).

${ }^{171}$ D. L. Kaiser, M. D. Vaudin, L. D. Rotter, Z. L. Wang, J. P. Cline, C. S. Hwang, R. B. Marinenko, and J. G. Gillen, Appl. Phys. Lett. 66, 2801 (1995)

${ }^{172}$ Z. Surowiak, V. M. Mukhortov, and V. P. Dudkevich, Ferroelectrics 139 1 (1993).

${ }^{173}$ S. Tsunekawa, T. Fukuda, T. Ozaki, Y. Yoneda, T. Okabe, and H. Terauchi, J. Appl. Phys. 84, 999 (1998).

${ }^{174}$ S. Tsunekawa, T. Fukuda, T. Ozaki, Y. Yoneda, and H. Terauchi, Appl. Phys. Lett. 71, 1486 (1997).

${ }^{175}$ S. K. Streifferet al., Phys. Rev. Lett. 89, 067601 (2002).

${ }^{176}$ S. K. Streifferet al., J. Appl. Phys. 83, 2742 (1998).

${ }^{177}$ A. E. Romanov, A. Vojta, W. Pompe, M. J. Lefevre, and J. S. Speck, Phys. Status Solidi A 172, 225 (1999).

${ }^{178}$ C. H. Lin, B. M. Yen, R. S. Batzer, and H. Chen, Ferroelectrics 221, 237 (1999).

${ }^{179}$ C. E. Zybillet al., Mater. Res. Soc. Symp. Proc. 541, 449 (1999).

${ }^{180}$ C. E. Zybill, B. Li, F. Koch, and T. Graf, Phys. Status Solidi A 177, 303 (2000).

${ }^{181}$ V. Gopalan and R. Raj, Appl. Phys. Lett. 68, 1323 (1996)

${ }^{182}$ V. Gopalan and R. Raj, J. Appl. Phys. 81, 865 (1997).

${ }^{183}$ Y. Barad, J. Lettieri, C. D. Theis, D. G. Schlom, V. Gopalan, J. C. Jiang, and X. Q. Pan, J. Appl. Phys. 89, 1387 (2001).

${ }^{184}$ A. Gruverman and Y. Ikeda, Jpn. J. Appl. Phys., Part 2 37, L939 (1998)

${ }^{185}$ M. A. Zurbuchen, G. Asayama, D. G. Schlom, and S. K. Streiffer, Phys. Rev. Lett. 88, 107601 (2002).

${ }^{186}$ A. L. Roytburd, Phys. Status Solidi A 37, 329 (1976).

${ }^{187}$ W. Pompe, X. Gong, Z. Suo, and J. S. Speck, J. Appl. Phys. 74, 6012 (1993).

${ }^{188}$ N. A. Pertsev and A. G. Zembilgotov, J. Appl. Phys. 78, 6170 (1995).

${ }^{189}$ A. E. Romanov, W. Pompe, and J. S. Speck, J. Appl. Phys. 79, 4037 (1996).

${ }^{190}$ V. G. Koukhar, N. A. Pertsev, and R. Waser, Phys. Rev. B 64, 214103 (2001)

${ }^{191}$ Y. L. Li, S. Y. Hu, Z. K. Liu, and L. Q. Chen, Appl. Phys. Lett. 78, 3878 (2001).

${ }^{192}$ A. L. Roytburd, J. Appl. Phys. 83, 239 (1998).

${ }^{193}$ A. L. Roytburd, in Thin Films Ferroelectric Material and Devices, edited by R. Ramesh (Kluwer Academic, Boston, 1997).

${ }^{194}$ N. A. Pertsev and V. G. Koukhar, Phys. Rev. Lett. 84, 3722 (2000)

${ }^{195}$ Y. L. Li, S. Choudhury, Z. K. Liu, and L. Q. Chen, Appl. Phys. Lett. 83, 1608 (2003)

${ }^{196}$ Y. L. Li, S. Y. Hu, Z. K. Liu, and L. Q. Chen, Acta Mater. 50, 395 (2002).

${ }^{197}$ A. K. Tagantsev (unpublished)

${ }^{198}$ K. Lee, K. S. Lee, and S. Baik, J. Appl. Phys. 90, 6327 (2001).

${ }^{199}$ A. M. Bratkovsky and A. P. Levanyuk, Phys. Rev. Lett. 80, 3177 (2000).

${ }^{200}$ A. Kopal, P. Mokry, J. Fousek, and T. Bahnik, Ferroelectrics 223, 127 (1999)

${ }^{201}$ G. B. Stephenson and K. R. Elder (unpublished).

${ }^{202}$ S. P. Alpay, V. Nagarajan, A. Bendersky, M. D. Vaudin, S. Aggarwal, R. Ramesh, and A. L. Roytburd, J. Appl. Phys. 85, 3271 (1999).

${ }^{203}$ S. P. Alpay and A. L. Roytburd, J. Appl. Phys. 83, 4714 (1998).

${ }^{204}$ L. S. J. Peng, X. X. Xi, B. H. Moeckly, and S. P. Alpay, Appl. Phys. Lett. 83, 4592 (2003).

${ }^{205}$ C. L. Canedy, H. Li, S. P. Alpay, L. Salamanca-Riba, A. L. Roytburd, and R. Ramesh, Appl. Phys. Lett. 77, 1695 (2000).

${ }^{206} \mathrm{H}$. Li, A. L. Roytburd, S. P. Alpay, T. D. Tran, L. Salamanca-Riba, and R. Ramesh, Appl. Phys. Lett. 78, 2354 (2001).

${ }^{207}$ A. K. Tagantsev, V. O. Sherman, K. F. Astafiev, J. Venkatesh, and N. Setter, J. Electroceram. 11, 5 (2003).

${ }^{208}$ J. H. Haeniet al., Nature (London) 430, 758 (2004).
${ }^{209}$ B. H. Park, E. J. Peterson, Q. X. Jia, J. Lee, X. Zeng, W. Si, and X. X. Xi, Appl. Phys. Lett. 78, 533 (2001).

${ }^{210}$ T. Yamada, K. F. Astafiev, V. O. Sherman, A. K. Tagantsev, P. Muralt, and N. Setter, Appl. Phys. Lett. 86, 142904 (2005).

${ }^{211}$ T. Yamada, K. F. Astafiev, V. O. Sherman, A. K. Tagantsev, P. Muralt, D. Su, and N. Setter, J. Appl. Phys. 98, 054105 (2005).

${ }^{212}$ J. Schubert, O. Trithaveesak, A. Petraru, C. L. Jia, R. Uecker, P. Reiche, and D. G. Schlom, Appl. Phys. Lett. 82, 3460 (2003).

${ }^{213}$ J. W. Matthews and A. E. Blakeslee, J. Cryst. Growth 27, 118 (1974).

${ }^{214}$ D.-W. Kim, D.-H. Kim, B.-S. Kang, T. W. Noh, D. R. Lee, and K. B. Lee, Appl. Phys. Lett. 74, 2176 (1999).

${ }^{215}$ T. Ohnishi, K. Takahashi, M. Nakamura, M. Kawasaki, M. Yoshimoto, and H. Koinuma, Appl. Phys. Lett. 74, 2531 (1999).

${ }^{216}$ A. Ohtomo and H. Y. Hwang, Nature (London) 427, 423 (2004).

${ }^{217}$ See, for instance, R. Kretschmer and K. Binder, Phys. Rev. B 20, 1065 (1979); D. R. Tilley and B. Zeks, Solid State Commun. 49, 823 (1984).

${ }^{218}$ M. D. Glinchuk, E. A. Eliseev, and V. A. Stephanovich, Physica B 322, 356 (2002).

${ }^{219}$ C. Zhou and D. M. Newns, J. Appl. Phys. 82, 3081 (1997).

${ }^{220}$ T. Mitsui and J. Furuichi, Phys. Rev. 90, 193 (1953).

${ }^{221}$ I. P. Batra, P. Wurfel, and B. D. Silverman, Phys. Rev. B 8, 3257 (1973).

${ }^{222}$ P. Wurfel, I. P. Batra, and J. T. Jacobs, Phys. Rev. Lett. 30, 1218 (1973).

${ }^{223}$ See, for instance, N. A. Pertsev and V. G. Koukhar, Phys. Rev. Lett. 84, $3722(2000)$

${ }^{224}$ Y. L. Li, S. Y. Hu, and L. Q. Chen, J. Appl. Phys. 97, 34112 (2005).

${ }^{225}$ See, for instance, K. Ishikawa, T. Nomura, N. Okada, and K. Takada, Jpn. J. Appl. Phys., Part 1 35, 5196 (1996).

${ }^{226}$ A. I. Kingon and S. Srinivasan, Nat. Mater. 4, 233 (2005).

${ }^{227}$ M. D. Losego, L. H. Jimison, J. F. Ihlefeld, and J. P. Maria, Appl. Phys. Lett. 86, 172906 (2005).

${ }^{228}$ R. W. Schwartz, Chem. Mater. 9, 2325 (1997).

${ }^{229}$ L. M. Doeswijk, G. Rijnders, and D. H. A. Blank, Appl. Phys. A: Mater. Sci. Process. A78, 263 (2004).

${ }^{230}$ D. A. Muller, N. Nakagawa, A. Ohtomo, J. L. Grazul, and H. Y. Hwang, Nature (London) 430, 657 (2004).

${ }^{231}$ A. Ohtomo, D. A. Muller, J. L. Grazul, and H. Y. Hwang, Nature (London) 419, 378 (2002).

${ }^{232}$ G. J. H. M. Rijnders, A. G. Koster, D. H. A. Blank, and H. Rogalla, Appl. Phys. Lett. 70, 1888 (1997).

${ }^{233}$ S. D. Bu, M. K. Lee, C. B. Eom, W. Tian, X. Q. Pan, S. K. Streiffer, and J. J. Krajewski, Appl. Phys. Lett. 79, 3482 (2001)

${ }^{234}$ T. Maeder, P. Muralt, and L. Sagalowicz, Thin Solid Films 345, 300 (1999).

${ }^{235}$ P. Muraltet al., J. Appl. Phys. 83, 3835 (1998).

${ }^{236}$ B. Pachaly, R. Bruchhaus, D. Pitzer, H. Huber, W. Wersing, and F. Koch, Integr. Ferroelectr. 5, 333 (1994).

${ }^{237}$ N. K. Pervez, P. J. Hansen, and R. A. York, Appl. Phys. Lett. 85, 4451 (2004).

${ }^{238}$ J. M. Trsicone, L. Frauchiger, M. Decroux, L. Mieville, O. Fischer, C. Beeli, P. Stadelmann, and G. A. Racine, J. Appl. Phys. 79, 4298 (1996).

${ }^{239}$ C. D. Theis, J. Yeh, D. G. Schlom, M. E. Hawley, and G. Brown, Thin Solid Films 325, 107 (1998).

${ }^{240}$ M. P. Warusawithana, E. V. Colla, J. N. Eckstein, and M. B. Weissman, Phys. Rev. Lett. 90, 036802 (2003).

${ }^{241}$ G. R. Bai, I.-F. Tsu, A. Wang, C. M. Foster, C. E. Murray, and V. P. Dravid, Appl. Phys. Lett. 72, 1572 (1998).

${ }^{242}$ M. de Keijser and G. J. M. Dormans, J. Cryst. Growth 149, 215 (1995).

${ }^{243}$ M. de Keijser and G. J. M. Dormans, MRS Bull. 21, 37 (1996).

${ }^{244}$ M. de Keijser, G. J. M. Dormans, J. F. M. Cillessen, D. M. de Leeuw, and H. W. Zandbergen, Appl. Phys. Lett. 58, 2636 (1991).

${ }^{245}$ G. J. M. Dormans, P. J. van Veldhoven, and M. de Keijser, J. Cryst. Growth 123, 537 (1992).

${ }^{246}$ Y. K. Kim, H. Morioka, R. Ueno, S. Yokoyama, and H. Funakubo, Appl. Phys. Lett. 86, 21290 (2005).

${ }^{247}$ B. S. Kwak, E. P. Boyd, and A. Erbil, Appl. Phys. Lett. 53, 1702 (1988).

${ }^{248}$ Z. Li, C. M. Foster, D. Guo, H. Zhang, G. R. Bai, P. M. Baldo, and L. E. Rehn, Appl. Phys. Lett. 65, 1106 (1994).

${ }^{249}$ M. V. R. Murty, S. K. Streiffer, G. B. Stephenson, J. A. Eastman, G. R. Bai, A. Munkholm, O. Auciello, and C. Thompson, Appl. Phys. Lett. 80, 1809 (2002)

${ }^{250}$ A. Nagai, H. Morioka, G. Asano, H. Funakubo, and A. Saiki, Appl. Phys. Lett. 86, 142906 (2005).

${ }^{251}$ M. Okada, S. Takai, M. Amemiya, and K. Tominaga, Jpn. J. Appl. Phys., Part 1 28, 1030 (1989) 
${ }^{252}$ J. F. Roeder, T. H. Baum, S. M. Bilodeau, G. T. Stauf, C. Ragaglia, M. W. Russell, and P. C. V. Buskirk, Adv. Mater. Opt. Electron. 10, 145 (2000).

${ }^{253}$ K. Saito, I. Yamaji, T. Akai, M. Mitsuya, and H. Funakubo, Jpn. J. Appl. Phys., Part 1 42, 539 (2003).

${ }^{254}$ C. H. Ahn, K. M. Rabe, and J. M. Triscone, Science 303, 488 (2004).

${ }^{255}$ M. Dawber, K. M. Rabe, and J. F. Scott, Rev. Mod. Phys. 77, 1083 (2005).

${ }^{256}$ T. M. Shaw, S. Trolier-McKinstry, and P. C. McIntyre, Annu. Rev. Mater. Sci. 30, 263 (2000).

${ }^{257}$ C. Bungaro and K. M. Rabe, Phys. Rev. B 71, 035420 (2005).

${ }^{258}$ R. E. Cohen, Nature (London) 358, 136 (1992).

${ }^{259}$ See, for instance, S. Tinte and M. Stachiotti, Phys. Rev. B 64, 235403 (2001).

${ }^{260}$ P. Ghosez and K. M. Rabe, Appl. Phys. Lett. 76, 2767 (2000).

${ }^{261}$ See, for example, C. B. Parker, J.-P. Maria, and A. I. Kingon, Appl. Phys. Lett. 81, 340 (2002).

${ }^{262}$ Note as well that similar results have been observed in $1 \mathrm{~nm}$ thick vinylidene fluoride / trifluoroethylene copolymer films. A. V. Bune, V. M. Fridkin, S. Ducharme, L. M. Blinov, S. P. Palto, A. V. Sorokin, S. G. Yudin, and A. Zlatkin, Nature (London) 391, 874 (1998).

${ }^{263}$ D. D. Fong, G. B. Stephenson, S. K. Streiffer, J. A. Eastman, O. Auciello, P. H. Fuoss, and C. Thompson, Science 304, 1650 (2004), and supplementary material.

${ }^{264}$ V. Nagarajanet al., Appl. Phys. Lett. 84, 5225 (2004).

${ }^{265}$ C. Lichtensteiger, J. M. Triscone, J. Junquera, and P. Ghosez, Phys. Rev. Lett. 94, 047603 (2005).

${ }^{266}$ J. Junquera and P. Ghosez, Nature (London) 422, 506 (2003).

${ }^{267}$ I. Kornev, H. Fu, and L. Bellaiche, Phys. Rev. Lett. 93, 196104 (2004)

${ }^{268}$ K. J. Choiet al., Science 306, 1005 (2004).

${ }^{269}$ H. Orihara, S. Hashimoto, and Y. Ishibashi, J. Phys. Soc. Jpn. 63, 1031 (1994).

${ }^{270}$ A. Kolmogorov, Izv. Akad. Nauk SSSR, Ser. Mat. 3, 355 (1937); M. Avrami, J. Chem. Phys. 8, 212 (1940).

${ }^{271}$ O. Lohse, M. Grossmann, U. Boettger, D. Bolten, and R. Waser, J. Appl. Phys. 89, 2332 (2001).

${ }^{272}$ A. Tagantsev, I. Stolichnov, N. Setter, J. Cross, and M. Tsukada, Phys. Rev. B 66, 214109 (2002).

${ }^{273}$ G. Gerra, A. K. Tagantsev, and N. Setter, Phys. Rev. Lett. 94, 107602 (2005).

${ }^{274}$ S. Hong, E. L. Colla, E. Kim, D. V. Taylor, A. K. Tagantsev, P. Muralt, K. No, and N. Setter, J. Appl. Phys. 86, 607 (1999).

${ }^{275}$ X. Du and I. Chen, Mater. Res. Soc. Symp. Proc. 493, 311 (1998).

${ }^{276}$ D. J. Jung, M. Dawber, J. F. Scott, L. J. Sinnamon, and J. M. Gregg, Integr. Ferroelectr. 48, 59 (2002).

${ }^{277}$ I. Stolichnov, A. Tagantsev, N. Setter, and J. Cross, Mater. Res. Soc. Symp. Proc. 784, C6.7 (2004).

${ }^{278}$ V. Nagarajan, C. S. Ganpule, and R. Ramesh, Ferroelectric Random Access Memories Fundamentals and Applications, edited by H. Ishiwara, M.Okuyama, and Y. Arimoto (Springer, New York, 2004), Vol. 93, p. 47.

${ }^{279}$ Y. W. So, D. J. Kim, T. W. Noh, J. G. Yoon, and T. K. Song, J. Korean Phys. Soc. 46, 40 (2005).

${ }^{280}$ E. Colla, S. Hong, D. Taylor, A. Tagantsev, K. No, and N. Setter, Appl. Phys. Lett. 72, 2763 (1998).

${ }^{281}$ A. Gruverman, O. Auciello, J. Hatano, and H. Takumoto, Ferroelectrics 184, 11 (1996).

${ }^{282}$ I. Stolichnov, L. Malin, E. Colla, A. K. Tagantsev, and N. Setter, Appl. Phys. Lett. 86, 012902 (2005).

${ }^{283}$ I. Stolichnov, A. Tagantsev, N. Setter, J. Cross, and M. Tsukada, Appl. Phys. Lett. 83, 3362 (2003).

${ }^{284}$ V. Nagarajan et al., Nat. Mater. 2, 43 (2002).

${ }^{285}$ A. Roelofs, N. A. Pertsev, R. Waser, F. Schlaphof, L. M. Eng, C. Ganpule, V. Nagarajan, and R. Ramesh, Appl. Phys. Lett. 80, 1424 (2002).

${ }^{286}$ I. Stolichnov, E. Colla, A. Tagantsev, S. S. N. Bharadwaja, H. Seungbum, N. Setter, J. S. Cross, and M. Tsukada, Appl. Phys. Lett. 80, 4804 (2002).

${ }^{287}$ W. Warren, B. Tuttle, D. Dimos, G. Pike, H. Al-Shareef, R. Ramesh, and J. Evans, Jpn. J. Appl. Phys., Part 1 35, 1521 (1998).

${ }^{288}$ D. Dimos, J. Appl. Phys. 76, 4305 (1994)

${ }^{289}$ M. Grossmann, O. Lhose, D. Bolten, U. Boettger, and R. Waser, Mater. Res. Soc. Symp. Proc. 688, C3.4.1 (2002).

${ }^{290}$ A. K. Tagantsev, I. Stolichnov, N. Setter, and J. S. Cross, J. Appl. Phys. 96, 6616 (2004)

${ }^{291}$ A. Tagantsev and I. Stolichnov, Appl. Phys. Lett. 74, 1326 (1999).

${ }^{292}$ M. Grossmann, O. Lhose, D. Bolten, U. Boettger, T. Schneller, and R. Waser, J. Appl. Phys. 92, 2680 (2002).
${ }^{293}$ M. Grossmann, O. Lhose, D. Bolten, U. Boettger, and R. Waser, J. Appl. Phys. 92, 2688 (2002).

${ }^{294}$ P. Schorn, U. Ellerkmann, D. Bolten, U. Boettger, and R. Waser, Integr. Ferroelectr. 53, 361 (2003).

${ }^{295}$ S. Traynor, T. Hadnagy, and L. Kammerdiner, Integr. Ferroelectr. 16, 63 (1997).

${ }^{296}$ A. Gruverman and M. Tanaka, J. Appl. Phys. 89, 1836 (2001).

${ }^{297}$ J. M. Benedetto, R. A. Moore, and F. B. Mclean, J. Appl. Phys. 75, 460 (1994).

${ }^{298}$ S. Sun and P. A. Fuierer, Integr. Ferroelectr. 23, 45 (1999).

${ }^{299}$ L. Kammerdiner, T. Davenport, and D. Hadnagy, US Patent No. 5969935 (19 October 1999).

${ }^{300}$ O. Auciello, Integr. Ferroelectr. 15, 211 (1997).

${ }^{301}$ C. A. az de Araujo, J. D. Cuchiaro, L. D. Mcmillan, M. C. Scott, and J. F. Scott, Nature (London) 374, 627 (1995).

${ }^{302}$ L. Goux et al., IEEE Trans. Electron Devices 52, 447 (2005).

${ }^{303}$ Z. G. Zhang, J. S. Liu, Y. N. Wang, J. S. Zhu, F. Yan, X. B. Chen, and H. M. Shen, Appl. Phys. Lett. 73, 788 (1998).

${ }^{304}$ P. Larsen, G. Dormans, D. Taylor, and P. Van-Veldhoven, J. Appl. Phys. 76, 2405 (1994).

${ }^{305}$ J. F. Scott and M. Dawber, Appl. Phys. Lett. 76, 3801 (2000).

${ }^{306}$ W. Warren, D. Dimos, B. Tuttle, G. Pike, R. Schwartz, P. Clew, and D. McIntyre, J. Appl. Phys. 77, 2623 (1995).

${ }^{307}$ E. Colla, A. Tagantsev, D. Taylor, and A. Kholkin, Integr. Ferroelectr. 18, 19 (1997)

${ }^{308}$ M. Dawber and J. F. Scott, Appl. Phys. Lett. 76, 1060 (2000).

${ }^{309}$ I. K. Yoo and S. B. Desu, Mater. Sci. Eng., B 13, 319 (1992).

${ }^{310}$ T. Nakamura, Y. Nakao, A. Kamisawa, and H. Takasu, Appl. Phys. Lett. 65, 1522 (1994).

${ }^{311}$ J. S. Cross, M. Fujiki, M. Tsukada, K. Matsuura, S. Otani, M. Tomotani, Y. Kataoka, Y. Kotaka, and Y. Goto, Integr. Ferroelectr. 25, 605 (1999).

${ }^{312}$ I. Stolichnov, A. Tagantsev, N. Setter, J. Cross, and M. Tsukada, Appl. Phys. Lett. 74, 3552 (1999).

${ }^{313}$ H. Al-Shareef, O. Auciello, and A. Kingon, J. Appl. Phys. 77, 2146 (1994).

${ }^{314}$ S. B. Majumder, B. Roy, R. S. Katiyar, and S. B. Krupanidhi, Integr. Ferroelectr. 39, 1077 (2001).

${ }^{315}$ Q. Zhang and R. W. Whatmore, Mater. Sci. Eng., B 109, 136 (2004).

${ }^{316}$ G. Binnig, C. F. Quate, and C. Gerber, Phys. Rev. Lett. 56, 930 (1986).

${ }^{317}$ L. M. Eng, M. Bammerlin, C. Loppacher, M. Guggisberg, R. Bennewitz, R. Lüthi, E. Meyer, and H. J. Güntherodt, Appl. Surf. Sci. 140, 253 (1999).

${ }^{318}$ M. Abplanalp, L. Eng, and P. Günter, Appl. Phys. A: Mater. Sci. Process. 66, S231 (1998).

${ }^{319}$ U. Zerweck, C. Loppacher, T. Otto, S. Grafström, and L. M. Eng, Phys. Rev. B 71, 125424 (2005)

${ }^{320}$ D. Pohl, W. Denk, and M. Lanz, Appl. Phys. Lett. 44, 651 (1984).

${ }^{321}$ K. Franke and M. Weihnacht, Ferroelectr., Lett. Sect. 19, 25 (1995).

${ }^{322}$ S. V. Kalinin and D. A. Bonnell, in Nanoscale Charaterisation of Ferroelectric Materials, edited by M. Alexe and A. Gruverman (Springer, New York, 2004), Chap. 2.

${ }^{323} \mathrm{C}$. Harnagea and A. Pignolet, in Nanoscale Charaterisation of Ferroelectric Materials, edited by M. Alexe and A. Gruverman (Springer, New York, 2004), Chap. 2.

${ }^{324}$ M. Abplanalp, M. Zgonik, and P. Guenter, in Nanoscale Charaterisation of Ferroelectric Materials, edited by M. Alexe and A. Gruverman (Springer, New York, 2004), Chap. 2.

${ }^{325}$ A. Roelofs, K. Szot, and R. Waser, in Nanoscale Phenomena in Ferroelectric Thin Films, edited by S. Hong (Kluwer, Dordrecht, 2004), Chap. 6.

${ }^{326}$ S. Hong and N. Setter, Appl. Phys. Lett. 81, 3437 (2002).

${ }^{327}$ S. Hong et al., J. Appl. Phys. 89, 1377 (2001).

${ }^{328} \mathrm{H}$. Shin, in Nanoscale Phenomena in Ferroelectric Thin Films, edited by S. Hong (Kluwer, Dordrecht, 2004), Chap. 11.

${ }^{329}$ H. Fujisawa and M. Shimizu, in Nanoscale Phenomena in Ferroelectric Thin Films, edited by S. Hong (Kluwer, Dordrecht, 2004), Chap. 9.

${ }^{330}$ P. Paruch, T. Tybell, and J. M. Triscone, Appl. Phys. Lett. 79, 530 (2001)

${ }^{331}$ J. Woo, S. Hong, D. K. Min, H. Shin, and K. No, Appl. Phys. Lett. 80, 4000 (2002).

${ }^{332}$ P. Paruch, T. Giamarchi, and J. M. Triscone, Phys. Rev. Lett. 94, 197601 (2005).

${ }^{333}$ S. V. Kalinin and D. A. Bonnell, in Nanoscale Phenomena in Ferroelectric Thin Films, edited by S. Hong (Kluwer, Dordrecht, 2004), Chap. 8. 
${ }^{334}$ S. Hong, B. Ecabart, E. L. Colla, and N. Setter, Appl. Phys. Lett. 84, 2382 (2004).

${ }^{335}$ O. Tikhomirov, B. Red'kin, A. Trivelli, and J. Levy, J. Appl. Phys. 87, 1932 (2000)

${ }^{336}$ A. V. Zayats and I. I. Smolyaninov, Philos. Trans. R. Soc. London, Ser. A 362, 843 (2004).

${ }^{337}$ J. Levy, C. Hubert, and A. Trivelli, J. Chem. Phys. 112, 7848 (2000).

${ }^{338}$ X. K. Orlik, M. Labardi, and M. Allegrini, Appl. Phys. Lett. 77, 2042 (2000).

${ }^{339}$ I. I. Smolyaninov, H. Y. Liang, C. H. Lee, and C. C. Davis, J. Appl. Phys. 89, 206 (2001).

${ }^{340}$ I. I. Smolyaninov, C. H. Lee, and C. C. Davis, Appl. Phys. Lett. 74, 1942 (1999).

${ }^{341}$ A. F. Xie, B. Y. Gu, G. Z. Yang, and Z. B. Zhang, Phys. Rev. B 63, 054104 (2001).

${ }^{342}$ I. I. Smolyaninov, H. Y. Liang, C. H. Lee, C. C. Davis, V. Nagarajan, and R. Ramesh, J. Microsc. 202, 250 (2001).

${ }^{343}$ T. J. Yang, V. Gopalan, P. J. Swart, and U. Mohideen, Phys. Rev. Lett. 82, 4106 (1999).

${ }^{344}$ T. Otto, J. Seidel, S. Grafström, and L. M. Eng, Appl. Phys. Lett. 84, $1168(2004)$

${ }^{345}$ T. Otto, S. Grafström, and L. M. Eng, Ferroelectrics 303, 149 (2004).

${ }^{346}$ C. Hubert, J. Levy, E. Cukauskas, and S. W. Kirchoefer, Integr. Ferroelectr. 29, 227 (2000).

${ }^{347}$ J. Renger, V. Deckert, I. Hellmann, S. Grafström, and L. M. Eng, J. Opt. Soc. Am. A 21, 1362 (2004).

${ }^{348}$ J. Renger, S. Grafström, L. M. Eng, and R. Hillenbrand, Phys. Rev. B 71, 075410 (2005).

${ }^{349}$ S. Schneider, S. Grafström, and L. M. Eng, Phys. Rev. B 71, 115418 (2005).

${ }^{350}$ H. Chaib, L. M. Eng, T. Otto, and S. Grafström, Phys. Rev. B 71, 085418 (2005).

${ }^{351}$ H. Chaib, F. Schlaphof, T. Otto, and L. M. Eng, J. Phys.: Condens. Matter 15, 8927 (2003).

${ }^{352}$ H. Chaib, T. Otto, and L. M. Eng, Phys. Status Solidi B 233, 250 (2002).

${ }^{353}$ H. Chaib, F. Schlaphof, T. Otto, and L. M. Eng, Ferroelectrics 291, 143 (2003).

${ }^{354}$ E. I. Bondarenko, V. Y. Topolov, and A. V. Turik, Ferroelectr., Lett. Sect. 13, 13 (1991).

${ }^{355}$ R. Herbeit, U. Robels, H. Dederichs, and G. Arlt, Ferroelectrics 98, 107 (1989).

${ }^{356}$ D. Damjanovic and M. Demartin, J. Phys.: Condens. Matter 9, 4943 (1997).

${ }^{357}$ D. A. Hall, J. Mater. Sci. 36, 4575 (2001).

${ }^{358}$ V. D. Kugel and L. E. Cross, J. Appl. Phys. 84, 2815 (1998).

${ }^{359}$ V. Mueller and Q. M. Zhang, Appl. Phys. Lett. 72, 2692 (1998).

${ }^{360}$ Q. M. Zhang, H. Wang, N. Kim, and L. E. Cross, J. Appl. Phys. 75, 454 (1994).

${ }^{361}$ D. Damjanovic, Rep. Prog. Phys. 61, 1267 (1998).

${ }^{362}$ L. Chen, V. Nagarajan, R. Ramesh, and A. L. Roytburd, J. Appl. Phys. 94, 5147 (2003)

${ }^{363}$ D. V. Taylor, D. Damjanovic, and N. Setter, Ferroelectrics 244, 299 (1999).

${ }^{364}$ D. V. Taylor and D. Damjanovic, Appl. Phys. Lett. 73, 2045 (1998).

${ }^{365}$ D. V. Taylor and D. Damjanovic, J. Appl. Phys. 82, 1973 (1997).

${ }^{366}$ D. Damjanovic and G. Robert, in Piezoelectric Materials for the end User, edited by N. Setter (Ceramics Laboratory, EPFL, Lausanne, Switzerland, 2002), Chap. 17, pp. 353-388.

${ }^{367}$ G. Robert, D. Damjanovic, N. Setter, and A. V. Turik, J. Appl. Phys. 89, 5067 (2001)

${ }^{368}$ V. V. Shvartsman, N. A. Pertsev, J. M. Herrero, C. Zaldo, and A. L. Kholkin, J. Appl. Phys. 97, 104105 (2005).

${ }^{369}$ S. Trolier-McKinstry, N. B. Gharb, and D. Damjanovic, Appl. Phys. Lett. 88, 202901 (2006).

${ }^{370}$ F. Xu, S. Trolier-McKinstry, W. Ren, and B. Xu, J. Appl. Phys. 89, 1336 (2001).

${ }^{371}$ S. E. Park and T. R. Shrout, J. Appl. Phys. 82, 1804 (1997)

${ }^{372}$ X. H. Du, J. Zheng, U. Belegundu, and K. Uchino, Appl. Phys. Lett. 72, 2421 (1998).

${ }^{373}$ D. V. Taylor and D. Damjanovic, Appl. Phys. Lett. 76, 1615 (2000).

${ }^{374}$ D. Damjanovic, J. Am. Ceram. Soc. 88, 2663 (2005).

${ }^{375}$ M. J. Haun, Z. Q. Zhuang, E. Furman, S. J. Jang, and L. E. Cross, Ferroelectrics 99, 45 (1989).

${ }^{376}$ M. Budimir, D. Damjanovic, and N. Setter, Appl. Phys. Lett. 85, 2890
(2004).

${ }^{377}$ M. Davis, D. Damjanovic, D. Hayem, and N. Setter, J. Appl. Phys. 98, 014102 (2005).

${ }^{378}$ Z. Kighelman, D. Damjanovic, M. Cantoni, and N. Setter, J. Appl. Phys. 91, 1495 (2002).

${ }^{379}$ Y. Zhang, I. S. Baturin, E. Aulbach, D. C. Lupascu, A. L. Kholkin, Y. V. Shur, and J. Rödel, Appl. Phys. Lett. 86, 012910 (2005).

${ }^{380}$ N. A. Pertsev, A. G. Zembilgotov, S. Hoffman, R. Waser, and A. K. Tagantsev, J. Appl. Phys. 85, 1698 (1999).

${ }^{381}$ N. A. Pertsev, A. G. Zembilgotov, and A. K. Tagantsev, Phys. Rev. Lett. 80, 1988 (1998).

${ }^{382}$ S. K. Streiffer, C. Basceri, C. B. Parker, S. E. Lash, and A. I. Kingon, J. Appl. Phys. 86, 4565 (1999).

${ }^{383}$ A. K. Tagantsev, N. A. Pertsev, P. Muralt, and N. Setter, Phys. Rev. B 65, 012104 (2002)

${ }^{384}$ Y. Yoneda, T. Okabe, K. Sakaue, and H. Terauchi, J. Appl. Phys. 83, 2458 (1998).

${ }^{385}$ N. A. Pertsev, G. Arlt, and A. G. Zembilgotov, Phys. Rev. Lett. 76, 1364 (1996).

${ }^{386}$ C. Basceri, S. K. Streiffer, A. I. Kingon, and R. Waser, J. Appl. Phys. 82, 2497 (1997)

${ }^{387}$ O. G. Vendik and S. P. Zubko, J. Appl. Phys. 88, 5343 (2000).

${ }^{388}$ A. M. Bratkovsky and A. P. Levanyuk, Phys. Rev. B 63, 132103 (2001).

${ }^{389}$ P. Mokry, A. K. Tagantsev, and N. Setter, Phys. Rev. B 70, 172107 (2004).

${ }^{390}$ A. M. Bratkovsky and A. P. Levanyuk, Phys. Rev. Lett. 94, 107601 (2005).

${ }^{391}$ O. G. Vendik, E. K. Hollmann, A. B. Kozyrev, and A. M. Prudan, J. Supercond. 12, 325 (1999).

${ }^{392}$ J. D. Baniecki, R. B. Laibowitz, T. M. Shaw, P. R. Duncombe, D. A. Neumayer, D. E. Kotecki, H. Shen, and Q. Y. Ma, Appl. Phys. Lett. 72, 498 (1998).

${ }^{393}$ S. Razumov et al., Integr. Ferroelectr. 39, 1317 (2001).

${ }^{394}$ A. K. Jonscher, Universal Relaxation Law (Chelsea Dielectrics, London, 1996).

${ }^{395}$ Y. Fukuda, K. Numata, K. Aoki, and A. Nishimura, Jpn. J. Appl. Phys., Part 1 35, 5178 (1996).

${ }^{396} \mathrm{R}$. Waser, in Science and Technology of Electroceramic Thin Films, edited by O. Auciello and R. Waser (Kluwer Academic, Norwell, MA, 1995), Vol. 284, p. 223

${ }^{397}$ O. G. Vendik and L. M. Platonova, J. Phys. Soc. Jpn. 28, 61 (1970).

${ }^{398}$ J. Petzelt et al., Phys. Rev. B 64, 184111 (2001).

${ }^{399}$ Y. Lemaitre, B. Marcilhac, D. Mansart, J. Siejka, and J. C. Mage, Physica C 372, 667 (2002).

${ }^{400}$ K. F. Astafiev, V. O. Sherman, A. K. Tagantsev, N. Setter, T. Kaydanova, and D. S. Ginley, Appl. Phys. Lett. 84, 2385 (2004).

${ }^{401}$ A. Tagantsev, Appl. Phys. Lett. 76, 1182 (2000).

${ }^{402}$ K. F. Astafiev, A. K. Tagantsev, and N. Setter, J. Appl. Phys. 97, 014106 (2005).

${ }^{403}$ S. Iigima, Nature (London) 354, 56 (1991).

${ }^{404}$ T. Kasuga, M. Hiramatsu, A. Hoson, T. Sekino, and K. Niihara, Langmuir 14, 3160 (1998).

${ }^{405}$ R. Tene, L. Margulis, and M. Genud, Nature (London) 360, 444 (1992).

${ }^{406}$ I. I. Naumov, L. Bellaiche, and H. Fu, Nature (London) 432, 737 (2004).

${ }^{407}$ E. J. Mele and P. Král, Phys. Rev. Lett. 88, 056803 (2002).

${ }^{408}$ S. M. Nakhmanson, A. Calzolari, V. Meunier, J. Bernholc, and M. B. Nardelli, Phys. Rev. B 67, 235406 (2003).

${ }^{409}$ Y. Luo et al., Appl. Phys. Lett. 83, 440 (2003).

${ }^{410}$ F. D. Morrison, L. Ramsay, and J. F. Scott, J. Phys.: Condens. Matter 15, L527 (2003)

${ }^{411}$ J. J. Urban, W. S. Yun, Q. Gu, and H. Park, J. Am. Ceram. Soc. 124, 1186 (2002).

${ }^{412}$ Y. Mao, S. Banerjee, and S. S. Wong, J. Am. Chem. Soc. 125, 15718 (2003)

${ }^{413}$ Y. Mao, S. Banerjee, and S. S. Wong, Chem. Commun. (Cambridge) 2003 , p. 408.

${ }^{414}$ J.-F. Liu, X.-L. Li, and Y.-D. Li, J. Nanosci. Nanotechnol. 2, 617 (2002).

${ }^{415}$ N. P. Padture and X. Wei, J. Am. Ceram. Soc. 86, 2215 (2003).

${ }^{416}$ E. Vasco, A. Magrez, L. Forro, and N. Setter, J. Phys. Chem. B 109, 14331 (2005)

${ }^{417}$ G. Suyal, E. Colla, R. Gysel, and N. Setter, Nano Lett. 4, 1339 (2004).

${ }^{418}$ W. S. Yun, J. J. Urban, Q. Gu, and H. Park, Nano Lett. 2, 447 (2002).

${ }^{419}$ H. Schmid, Ferroelectrics 162, 317 (1994).

${ }^{420}$ E. Ascher, H. Rieder, H. Schmid, and H. Stössel, J. Appl. Phys. 37, 1404 
(1966).

${ }^{421}$ G. A. Smolenskii, V. A. Isupov, and A. I. Agranovskaya, Sov. Phys. Solid State 1, 149 (1959).

${ }^{422}$ G. A. Smolenskii and I. E. Chupis, Sov. Phys. Usp. 25, 475 (1982).

${ }^{423}$ J. Wang et al., Science 299, 1719 (2003).

${ }^{424}$ C. Ederer and N. A. Spaldin, Phys. Rev. B 71, 224103 (2005).

${ }^{425}$ W. Eerenstein, F. D. Morrison, J. Dho, M. G. Blamire, J. F. Scott, and N. D. Mathur, Science 307, 1203 (2005).

${ }^{426}$ J. B. Neaton, C. Ederer, U. V. Waghmare, N. A. Spaldin, and K. M. Rabe, Phys. Rev. B 71, 014113 (2005).

${ }^{427}$ J. Wang et al., Science 307, 1203b (2005).

${ }^{428}$ K. Y. Yun, M. Noda, and M. Okuyama, Appl. Phys. Lett. 83, 3981 (2003).

${ }^{429}$ B. K. Ponomarev, S. A. Ivanov, Yu. F. Popov, V. D. Negril, and B. S. Red'kin, Ferroelectrics 161, 43 (1994).

${ }^{430}$ N. Hur, S. Park, P. A. Sharma, J. S. Ahn, S. Guha, and S.-W. Cheong, Nature (London) 429, 392 (2004).

${ }^{431}$ T. Kimura, T. Goto, H. Shintani, K. Ishizaka, T. Arima, and Y. Tokura, Nature (London) 426, 55 (2003).

${ }^{432}$ N. Hill, J. Phys. Chem. B 104, 6694 (2000).

${ }^{433}$ J. M. Gregg, J. Phys.: Condens. Matter 15, V11 (2003), and references therein.

${ }^{434}$ A. Q. Jiang, J. F. Scott, H. Lu, and Z. Chen, J. Appl. Phys. 93, 1180 (2003).

${ }^{435}$ J. C. Jiang, X. Q. Pan, W. Tian, C. D. Theis, and D. G. Schlom, Appl. Phys. Lett. 74, 2851 (1999).

${ }^{436}$ H. N. Lee, H. M. Christen, M. F. Chisholm, C. M. Rouleau, and D. H. Lowndes, Nature (London) 433, 395 (2005).

${ }^{437}$ See S. Dong, J.-F. Li, and D. Viehland, Appl. Phys. Lett. 83, 2265 (2003); G. Srinivasan, E. T. Tasmussen, A. A. Bush, K. E. Kamentsev, V. F. Meshcheryakov, and Y. K. Fetisov, Appl. Phys. A: Mater. Sci. Process.
78, 721 (2004), and references therein.

${ }^{438}$ H. Zheng et al., Science 303, 661 (2004).

${ }^{439}$ J. H. Li, I. Levin, J. Slutsker, V. Provenzano, P. K. Schenck, R. Ramesh, J. Ouyang, and A. L. Roytburd, Appl. Phys. Lett. 87, 072909 (2005).

${ }^{440}$ F. Zavaliche et al., Nano Lett. 5, 1793 (2005).

${ }^{441}$ T. Zhao, S. R. Shinde, S. B. Ogale, H. Zheng, T. Venkatesan, R. Ramesh, and S. D. Sarma, Phys. Rev. Lett. 94, 126601 (2005).

${ }^{442}$ N. A. Pertsev, A. K. Tagantsev, and N. Setter, Phys. Rev. B 61, R825 (2000).

${ }^{443}$ Numerical Data and Functional Relationships in Science and Technology, Landolt-Bornstein New series, Vol. III/29a,b (Springer, New York, 1981).

${ }^{444}$ H. Uwe and T. Sakudo, Phys. Rev. B 13, 271 (1976).

${ }^{445} \mathrm{~S}$. Trolier-McKinstry and P. Muralt, in Electroceramic Based MEMS, edited by N. Setter (Springer, New York, 2005), p. 199.

${ }^{446}$ K. S. Lee, J. H. Choi, J. Y. Lee, and S. Baik, J. Appl. Phys. 90, 4095 (2001).

${ }^{447}$ D. V. Taylor, Ph.D. thesis Swiss Federal Institute of Technology (EPFL), 1999.

${ }^{448}$ In the case where the substrate is pseudocubic.

${ }^{449}$ The following relations correspond to those from Ref. 383 to within the difference in the definition of $Q_{44}$.

${ }^{450}$ In the case of $\mathrm{SrTiO}_{3}$, the whole set of data for $Q$ 's is contradictory; a self-consistent subset of these data has been used in calculations. A discussion of the inaccuracy of the evaluation of strain effects on the permittivity of $\mathrm{SrTiO}_{3}$ can be found in Ref. 208. In the case of $\mathrm{SrTiO}_{3}$, Eqs. (7)-(9) are applicable for temperatures higher than $100 \mathrm{~K}$, and for lower temperatures the effect of the quantum statistics should be taken into account (see Refs. 208 and 442 for a more detailed discussion). There may also be small corrections associated with the structure phase transition. 